Supporting Information

\title{
The Preussochromone Puzzle: Structural Revision of Preussochromone E \& F by Total Synthesis
}

\author{
Marc Paul Beller ${ }^{\dagger}$, Sergei Ivlev ${ }^{\dagger}$, Ulrich Koert ${ }^{\dagger *}$ \\ $\dagger$ Department of chemistry, Philipps-Universität Marburg, Hans-Meerwein-Strasse 4, D-35043 \\ Marburg, Germany \\ koert@chemie.uni-marburg.de
}

Experimental procedures, spectroscopic and analytical data

of all new compounds 


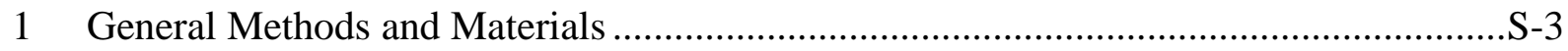

2 Synthetic Procedures for the Preparation of Preussochromone D (3) ...........................S-5

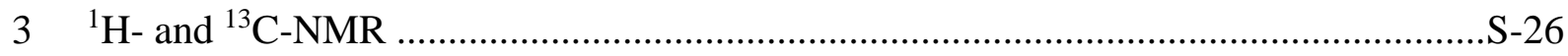

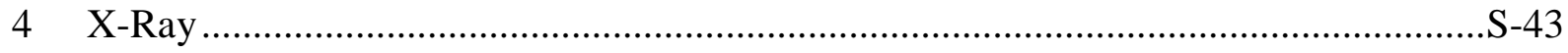

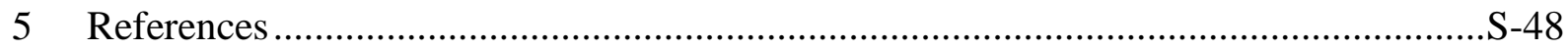




\section{General Methods and Materials}

All non-aqueous reactions were carried out using flame-dried glassware under argon atmosphere. All solvents were distilled by rotary evaporation. Solvents for non-aqueous reactions were dried as follows prior to use: THF and toluene were distilled from Solvona ${ }^{\circledR}$. $\mathrm{CH}_{2} \mathrm{Cl}_{2}, \mathrm{MeCN}$ and $\mathrm{NEt}_{3}$ were distilled from $\mathrm{CaH}_{2} . \mathrm{MeOH}$ and DMF were purchased from Acros Organics in extra dry quality and stored under an argon atmosphere over MS 4 A. All commercially available reagents and reactants were used without purification unless otherwise noted.

2-Azido-1,3-dimethylimidazolinium hexafluorophosphate (ADMP) was synthesized by the procedure of Kitamura and Murakami. ${ }^{[1]}$

Reactions were monitored by thin layer chromatography (TLC) using Merck Silica Gel $60 \mathrm{~F}_{254-}$ plates and visualized by fluorescence quenching under UV-light. In addition, TLC-plates were stained either using a ceric sulfate/phosphomolybdic acid or $\mathrm{KMnO}_{4}$ stain. Chromatographic purification of products was performed on Merck Silica Gel 60 (230-400 mesh) unless otherwise noted using a forced flow of eluents. Concentration under reduced pressure was performed by rotary evaporation at $45{ }^{\circ} \mathrm{C}$ and appropriate pressure and by exposing to fine vacuum at room temperature if necessary.

NMR spectra were recorded on a Bruker AV 300 MHz, AV III 500 MHz, AV III HD 500 MHz spectrometer at room temperature. Chemical shifts are reported in ppm with the solvent resonance as internal standard. Data are reported as follows: $\mathrm{s}=\operatorname{singlet}, \mathrm{d}=\mathrm{doublet}, \mathrm{t}=$ triplet, $\mathrm{q}=$ quartet, quint $=$ quintet, $\mathrm{m}=$ multiplet.

Mass spectra were recorded by the mass service department of the Philipps-Universität Marburg. HR-ESI mass spectra were acquired with an LTQ-FT mass spectrometer (Thermo Fischer Scientific). The resolution was set to 100000 .

IR spectra were recorded on a Bruker IFS 200 spectrometer. The absorption bands are given in wave numbers $\left(\mathrm{cm}^{-1}\right)$, intensities are reported as follows: $\mathrm{s}=$ strong, $\mathrm{m}=$ medium, $\mathrm{w}=$ weak, br $=$ broad band.

Melting points were determined on a Mettler Toledo MP70 using one end closed capillary tubes.

Diastereomeric ratios were accurately determined by integration of ${ }^{1} \mathrm{H}-\mathrm{NMR}$ spectra. 
Optical rotations were determined at $20{ }^{\circ} \mathrm{C}$ for the Na-D wavelength $(589 \mathrm{~nm})$ with a Krüss P8000 T polarimeter.

Room temperature was $21-24{ }^{\circ} \mathrm{C}$. 


\section{Synthetic Procedures for the Preparation of Preussochromone E \& F (23 \& 24)}

Note: Diene 8 was synthesized by our previously published procedure, but using the L-Menthol instead of D-Menthol derived ketene acetal 9. ${ }^{[2]}$ Only exception in the route is the methylenation which was previously realized by using Takai-conditions $\left(\mathrm{CH}_{2} \mathrm{I}_{2}, \mathrm{Ti}(\mathrm{OiPr})_{4}, \mathrm{Zn}\right)$. We switched to Nysted-Reagent/BF $3 \cdot \mathrm{OEt}_{2}$ as it was easier to reproduce on larger scale $(\sim 13 \mathrm{mmol})$.

\section{1 (S)-2-((S)-1-hydroxypropan-2-yl)-5-methoxychroman-4-one (11)}

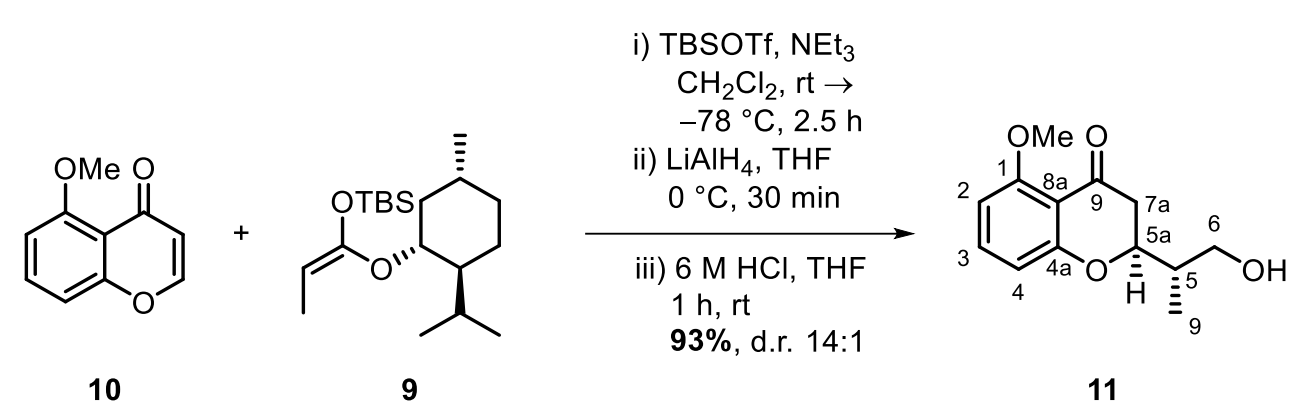

Chromenone 10 (1.54 g, 8.74 mmol, 1.00 equiv) was dissolved in $\mathrm{CH}_{2} \mathrm{Cl}_{2}$ (75 mL) and TBSOTf ( $2.50 \mathrm{~mL}, 13.1 \mathrm{mmol}, 1.50$ equiv) was added at rt. The deep yellow solution was stirred for $1 \mathrm{~h}$ and then cooled to $-78{ }^{\circ} \mathrm{C}$. A solution of 9 (5.14 g, $15.7 \mathrm{mmol}, 1.80$ equiv) in $\mathrm{CH}_{2} \mathrm{Cl}_{2}(30 \mathrm{~mL})$ was added slowly over $1 \mathrm{~h}$ using a syringe pump. After stirring for another $30 \mathrm{~min}, \mathrm{NEt}_{3}$ ( $2.42 \mathrm{~mL}, 17.5 \mathrm{mmol}, 2.00$ equiv) was added to the deep red solution and it was slowly warmed to rt. $\mathrm{MeOH}(1.5 \mathrm{~mL})$ was added and the now deep yellow mixture was concentrated in vacuo. The residue was redissolved in THF $(75 \mathrm{~mL})$ and cooled to $0{ }^{\circ} \mathrm{C}$. $\mathrm{LiAlH}_{4}(1.66 \mathrm{~g}, 43.7 \mathrm{mmol}$, 5.00 equiv) was slowly added (intense foaming!) and the suspension was stirred at $0{ }^{\circ} \mathrm{C}$ for 30 min. The reaction was quenched by careful and slow addition of $\mathrm{MeOH}(15 \mathrm{~mL})$ and then $6 \mathrm{M}$ $\mathrm{HCl}(15 \mathrm{~mL})$. The suspension was stirred for $1 \mathrm{~h}$ at $\mathrm{rt}$, after which aq. sat. K/Na-tartrate was added $(200 \mathrm{~mL})$. The aqueous layer was extracted with EtOAc $(3 \times 150 \mathrm{~mL})$ and the combined organic layers were washed with brine, dried over $\mathrm{MgSO}_{4}$, filtered and all volatiles were removed in vacuo. The crude product was purified by flash chromatography on silica (1:3 npentane/EtOAc) to afford alcohol $11(1.93 \mathrm{~g}, 8.17 \mathrm{mmol}, 93 \%)$ as beige solid.

TLC: $\mathrm{R}_{f}=0.32$ (EtOAc). ${ }^{1} \mathbf{H}-N M R:\left(500 \mathrm{MHz}, \mathrm{CDCl}_{3}\right) \delta=7.38(\mathrm{t}, J=8.4 \mathrm{~Hz}, 1 \mathrm{H}, \mathrm{H}-3), 6.59$ (dd, $J=8.4,1.0 \mathrm{~Hz}, 1 \mathrm{H}, \mathrm{H}-4), 6.52$ (dd, $J=8.4,0.9 \mathrm{~Hz}, 1 \mathrm{H}, \mathrm{H}-2), 4.56$ (ddd, $J=13.7,4.3,2.6$ Hz, 1H, H-5a), 3.92 (s, 3H, - $\mathrm{OCH}_{3}$ ), $3.84-3.77$ (m, 1H, H-6), 3.73 (dt, J=10.4, 4.7 Hz, 1H, H-6'), 2.86 (dd, $J=16.2,13.7 \mathrm{~Hz}, 1 \mathrm{H}, \mathrm{H}-7 \mathrm{a}), 2.58$ (dd, $J=16.2,2.6 \mathrm{~Hz}, 1 \mathrm{H}, \mathrm{H}-7 \mathrm{a}$ '), 2.10 1.99 (m, 1H, H-5), 1.84 (t, $J=4.6 \mathrm{~Hz}, 1 \mathrm{H}, 6-\mathrm{OH}), 1.10$ (d, $J=7.0 \mathrm{~Hz}, 3 \mathrm{H}, \mathrm{H}-9)$ ppm. ${ }^{13} \mathrm{C}-$ 
NMR: (126 MHz, $\left.\mathrm{CDCl}_{3}\right) \delta=191.7$ (C-8), 163.4 (C-4a), 160.9 (C-1), 135.9 (C-3), 111.5 (C8a), 110.1 (C-4), 104.0 (C-2), 78.4 (C-5a), 64.6 (C-6), $56.3\left(-\mathrm{OCH}_{3}\right), 42.0$ (C-7a), 39.4 (C-5), 11.4 (C-9) ppm. HRMS (ESI+): $\mathrm{m} / \mathrm{z}$ calc. for $\mathrm{C}_{13} \mathrm{H}_{16} \mathrm{O}_{4} \mathrm{Na}[\mathrm{M}+\mathrm{Na}]^{+}:$259.0941, found 259.0941. FT-IR: (neat): $\tilde{v}=3474$ (w), 2966 (w), 2936 (w), 2883 (w), 1675 (s), 1601 (s), 1574 (m), 1471 (s), 1442 (w), 1382 (w), 1332 (m), 1258 (s), 1186 (w), 1103 (w), 1084 (s), 1034 (m), $991(\mathrm{w}), 944(\mathrm{w}), 916(\mathrm{w}), 888(\mathrm{w}), 790(\mathrm{~m}), 736(\mathrm{w}), 642(\mathrm{w}), 617(\mathrm{w}), 583(\mathrm{w}), 495(\mathrm{w}) \mathrm{cm}^{-}{ }^{1}$. m.p.: $65{ }^{\circ} \mathrm{C}\left(\mathrm{Et}_{2} \mathrm{O}\right) .[\boldsymbol{\alpha}]_{\mathrm{D}}^{20}:+81.0\left(\mathrm{c} 0.5, \mathrm{CHCl}_{3}\right)$.

\section{2 (S)-2-((S)-But-3-en-2-yl)-5-methoxychroman-4-one (12)}

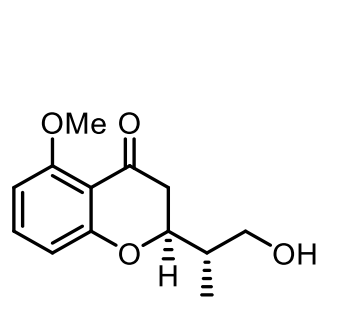

11
1) IBX, EtOAc reflux, $7.5 \mathrm{~h}$

2) NYSTED Reagent $\mathrm{BF}_{3} \cdot \mathrm{OEt}_{2}$, THF $0^{\circ} \mathrm{C} \rightarrow \mathrm{rt}, 16 \mathrm{~h}$

$\mathbf{6 5 \%}$ over two steps

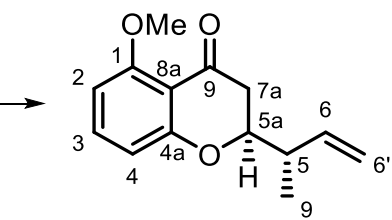

12

Alcohol 11 (3.00 g, 12.7 mmol, 1.00 equiv) was dissolved in EtOAc (318 mL) and IBX (7.11 g, $25.4 \mathrm{mmol}, 2.00$ equiv) was added in one portion. The suspension was heated to reflux and stirred for $7.5 \mathrm{~h}$. The resulting suspension was cooled to $0{ }^{\circ} \mathrm{C}$ and $\mathrm{Et}_{2} \mathrm{O}(318 \mathrm{~mL})$ was added. After stirring for $30 \mathrm{~min}$, the suspension was filtered through a pad of $\mathrm{Na}_{2} \mathrm{SO}_{4}$ and the solvent was removed in vacuo to give the aldehyde as an orange oil. In a separate flask, Nysted-Reagent (86.9 $\mathrm{g}$ of a $20 \mathrm{wt} \%$ suspension in THF, $38.1 \mathrm{mmol}, 3.00$ equiv) was diluted with THF (100 mL) and cooled to $0{ }^{\circ} \mathrm{C} . \mathrm{BF}_{3} \cdot \mathrm{OEt}_{2}(4.83 \mathrm{~mL}, 38.1 \mathrm{mmol}, 3.00$ equiv) and a solution of the freshly prepared aldehyde in THF $(15 \mathrm{~mL})$ were added successively and the suspension was allowed to warm to rt overnight. The reaction was quenched by the careful addition of aq. sat. $\mathrm{NaHCO}_{3}$ ( $250 \mathrm{~mL})$ and $\mathrm{H}_{2} \mathrm{O}(100 \mathrm{~mL})$ at $0{ }^{\circ} \mathrm{C}$ and the suspension was vigorously stirred for $15 \mathrm{~min}$. The mixture was filtered through a glass frit and the filtrate was extracted with EtOAc $(3 \times 200 \mathrm{~mL})$. The combined organic layers were washed with $\mathrm{H}_{2} \mathrm{O}$ and brine, dried over $\mathrm{MgSO}_{4}$, filtered and concentrated in vacuo. Purification by flash chromatography on silica (3:1 n-pentane/EtOAc) afforded alkene 12 (1.91 g, $8.22 \mathrm{mmol}, 65 \%)$ as pale yellow oil.

TLC: $\mathrm{R}_{f}=0.43\left(2: 1 n\right.$-pentane/EtOAc). ${ }^{1} \mathbf{H}$-NMR: $\left(500 \mathrm{MHz}, \mathrm{CDCl}_{3}\right) \delta=7.36(\mathrm{t}, J=8.3 \mathrm{~Hz}$, $1 \mathrm{H}, \mathrm{H}-3), 6.59$ (dd, $J=8.3,1.0 \mathrm{~Hz}, 1 \mathrm{H}, \mathrm{H}-4), 6.49$ (dd, $J=8.4,1.0 \mathrm{~Hz}, 1 \mathrm{H}, \mathrm{H}-2), 5.78$ (ddd, $J$ = 17.2, 10.4, 7.8 Hz, 1H, H-6), 5.17 - 5.13 (m, 1H, H-6'), 5.13 - 5.11 (m, 1H, H-6'), 4.20 (ddd, $J=11.3,7.0,4.2 \mathrm{~Hz}, 1 \mathrm{H}, \mathrm{H}-5 \mathrm{a}), 3.90\left(\mathrm{~s}, 3 \mathrm{H},-\mathrm{OCH}_{3}\right), 2.71-2.55$ (m, 3H, H-7a, H-5), 1.17 (d, $J=6.9 \mathrm{~Hz}, 3 \mathrm{H}, \mathrm{H}-9)$ ppm. ${ }^{13} \mathrm{C}-\mathbf{N M R}:\left(125 \mathrm{MHz}, \mathrm{CDCl}_{3}\right) \delta=191.6(\mathrm{C}-8), 163.4$ (C-4a), 160.7 (C-1), 138.5 (C-6), 135.9 (C-3), 116.7 (C-6’), 111.6 (C-8a), 110.2 (C-4), 103.8 (C-2), 80.4 
(C-5a), $56.3\left(-\mathrm{OCH}_{3}\right), 42.1$ (C-5), 42.1 (C-7a), 15.6 (C-9) ppm. HRMS (ESI+): m/z calc. for $\mathrm{C}_{14} \mathrm{H}_{17} \mathrm{O}_{3}[\mathrm{M}+\mathrm{H}]^{+}:$233.1172, found 233.1173. FT-IR: (neat): $\tilde{v}=3078$ (w), 2971 (w), 2934 (w), 2841 (w), 1682 (s), 1642 (w), 1599 (s), 1574 (m), 1470 (s), 1441 (w), 1377 (w), 1330 (m), $1280(\mathrm{w}), 1252$ (s), 1226 (w), 1171 (w), 1100 (w), 1082 (s), 1049 (w), 996 (w), 919 (m), 886 (w), $855(\mathrm{w}), 786(\mathrm{~m}), 736(\mathrm{~m}), 678(\mathrm{w}), 654(\mathrm{w}), 584(\mathrm{~m}), 556(\mathrm{w}), 520(\mathrm{w}), 487(\mathrm{w}), 428(\mathrm{w})$ $\mathrm{cm}^{-1} \cdot[\boldsymbol{\alpha}]_{\mathbf{D}}^{20}:+96.5\left(\mathrm{c} 0.5, \mathrm{CHCl}_{3}\right)$.

\section{$2.3 \quad(((R)-2-((S)$-But-3-en-2-yl)-5-methoxy-2H-chromen-4-yl)oxy $)($ tert-} butyl)dimethylsilane (13)<smiles>C=C[C@H](C)[C@H]1CC(=O)c2c(OC)cccc2O1</smiles>

12

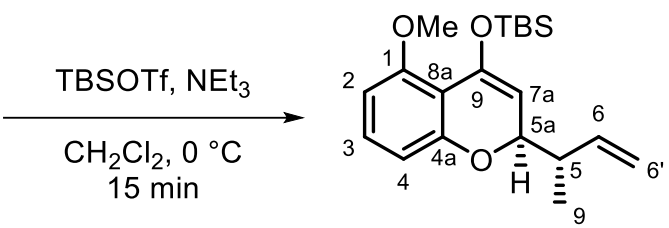

13

Alkene 12 (4.77 g, 20.5 mmol, 1.00 equiv) was dissolved in $\mathrm{CH}_{2} \mathrm{Cl}_{2}(200 \mathrm{~mL}$ ) and TBSOTf (5.66 mL, $24.6 \mathrm{mmol}, 1.20$ equiv) and $\mathrm{NEt}_{3}(3.41 \mathrm{~mL}, 24.6 \mathrm{mmol}, 1.20$ equiv) were added subsequently at $0{ }^{\circ} \mathrm{C}$. The yellow solution was stirred for $15 \mathrm{~min}$ at $0{ }^{\circ} \mathrm{C}$ and was then quenched by addition of $\mathrm{H}_{2} \mathrm{O}(200 \mathrm{~mL})$. The layers were separated and the aqueous layer was extracted with $\mathrm{CH}_{2} \mathrm{Cl}_{2}$ (3x $150 \mathrm{~mL}$ ). The combined organic layers were dried over $\mathrm{MgSO}_{4}$, filtered and all volatiles were removed in vacuo. The crude product was filtered through a silica column and eluted with 20:1 n-pentane/EtOAc. The solvent was removed in vacuo and the silyl enol ether 13 was used without further purification.

Note: Silyl enol ether 13 is not soluble in 20:1 n-pentane/EtOAc. Additional, little amounts of EtOAc should be used to get it in solution and on the column. It runs very fast and therefore can contain little amounts of hydrolyzed TBSOTf-byproduct which does not interfere in the next reaction.

\subsection{Methyl $(R)-2-((2 R, 3 R)-2-((S)-b u t-3-e n-2-y l)-5-m e t h o x y-4-0 x o c h r o m a n-3-y l)-$ 2-(phenylthio)propanoate (15a)}<smiles>C=C[C@H](C)[C@H]1C=C([O+])c2c(OC)cccc2O1</smiles>

13

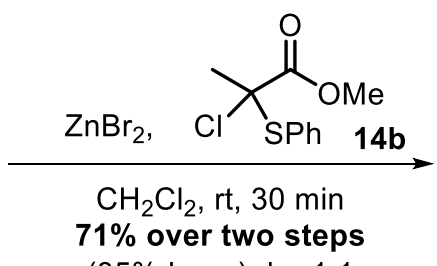

(85\% brsm) d.r. $1: 1$

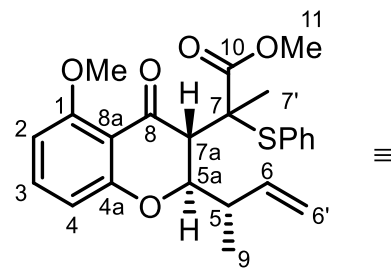

15

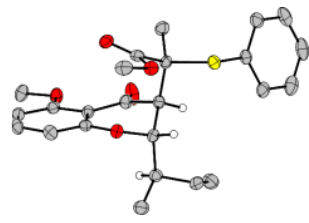

ent-15a 
Silyl enol ether 13 (1.40 g, 4.04 mmol, 1.00 equiv) and freshly prepared 14b (1.59 g, 8.08 mmol, 2.00 equiv) were dissolved in $\mathrm{CH}_{2} \mathrm{Cl}_{2}$ (88.5 mL). $\mathrm{ZnBr}_{2}$ (591 mg, $2.63 \mathrm{mmol}, 0.50$ equiv) was added in one portion upon which the solution turned dark green. The color of the solution changed to red after stirring for $30 \mathrm{~min}$ at rt, upon which silica was added and all volatiles were removed in vacuo. The crude products adsorbed on silica were purified by flash chromatography on silica (20:1 $n$-pentane/EtOAc) to afford sulfide 15a (583 mg, $1.37 \mathrm{mmol}$, 34\%), alkene 12 (126 mg, $0.54 \mathrm{mmol}, 14 \%$ ) and sulfide 15b (639 mg, $1.50 \mathrm{mmol}, 37 \%$ ). A suitable crystal of ent-15a for structure determination was obtained by cooling a concentrated $\mathrm{Et}_{2} \mathrm{O}$ solution in a small vial standing in a bigger vial filled with $n$-pentane to $-20{ }^{\circ} \mathrm{C}$ for 2 days. First eluting diastereomere 15a (colorless solid):

TLC: $\mathrm{R}_{f}=0.47$ (3:1 $n$-pentane/EtOAc). ${ }^{1} \mathbf{H}$-NMR: $\left(500 \mathrm{MHz}, \mathrm{CDCl}_{3}\right) \delta=7.64-7.60(\mathrm{~m}, 2 \mathrm{H}$, $\mathrm{Ar}_{\text {ortho }}$ ), $7.40-7.35$ (m, 2H, H-3, Ar $\left.\mathrm{p}_{\text {para }}\right), 7.33-7.28$ (m, 2H, $\mathrm{Ar}_{\text {meta }}$ ), 6.53 - 6.49 (m, 2H, H-2, H-4), 5.48 (ddd, $J=17.1,10.1,9.0$ Hz, 1H, H-6), 5.09 - 5.05 (m, 1H, H-6'), 5.07 - 5.01 (m, 1H, H-6'), 4.25 (d, J = 10.4 Hz, 1H, H-5a), 3.94 (s, 3H, -OCH ), 3.55 (s, 3H, C-11), 3.27 (d, J $=0.8 \mathrm{~Hz}, 1 \mathrm{H}, \mathrm{H}-7 \mathrm{a}), 2.65-2.53$ (m, 1H, H-5), 1.48 (s, 3H, H-7'), 1.04 (d, J=6.6 Hz, 3H, H-9) ppm. ${ }^{13}$ C-NMR: (126 MHz, $\left.\mathrm{CDCl}_{3}\right) \delta=188.8$ (C-8), 171.7 (C-10), 160.7 (C-1), 160.3 (C-4a), 138.4 (C-6), 138.0 (2C, $\mathrm{Ar}_{\text {ortho }}$ ), 136.4 (C-3), 130.6 ( $\left.\operatorname{Ar}_{\text {ipso }}\right), 129.9$ ( $\left.\operatorname{Ar}_{\text {para }}\right), 128.7$ (2C, $\left.\operatorname{Ar}_{\text {meta }}\right)$,

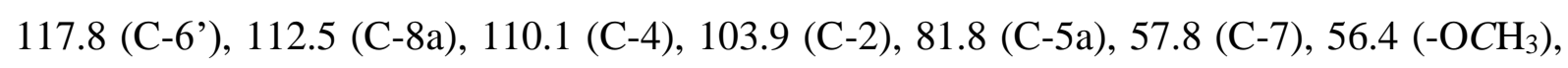
52.6 (C-7a), 52.4 (C-11), 41.2 (C-5), 20.6 (C-7'), 17.5 (C-9) ppm. HRMS (ESI+): m/z calc. for $\mathrm{C}_{24} \mathrm{H}_{26} \mathrm{O}_{5} \mathrm{SNa}[\mathrm{M}+\mathrm{Na}]^{+}:$449.1404, found 449.1416. FT-IR: (neat): $\tilde{v}=2951(\mathrm{w}), 1728$ (s), 1674 (s), 1600 (s), 1577 (w), 1470 (s), 1432 (w), 1376 (w), 1348 (w), 1323 (w), 1266 (w), 1245 (s), $1222(\mathrm{w}), 1206(\mathrm{w}), 1170(\mathrm{w}), 1148(\mathrm{w}), 1087$ (s), $1030(\mathrm{w}), 1002(\mathrm{w}), 986(\mathrm{w}), 968(\mathrm{w})$, $939(\mathrm{w}), 918(\mathrm{~m}), 848(\mathrm{w}), 829(\mathrm{~m}), 776(\mathrm{w}), 761(\mathrm{~s}), 738(\mathrm{w}), 705(\mathrm{~m}), 679(\mathrm{w}), 650(\mathrm{w}), 578$ (w), $535(\mathrm{w}), 500(\mathrm{~m}), 457(\mathrm{w}) \mathrm{cm}^{-1}$. m.p.: $95^{\circ} \mathrm{C}\left(\mathrm{Et}_{2} \mathrm{O}\right) . \quad[\boldsymbol{\alpha}]_{\mathrm{D}}^{20}:+98.4\left(\mathrm{c} 0.5, \mathrm{CHCl}_{3}\right)$.

Second eluting diastereomere $\mathbf{1 5 b}$ (pale yellow, sticky semi-solid):

TLC: $\mathrm{R}_{f}=0.23$ (3:1 $n$-pentane/EtOAc). ${ }^{1} \mathbf{H}$-NMR: $\left(500 \mathrm{MHz}, \mathrm{CDCl}_{3}\right) \delta=7.50-7.46(\mathrm{~m}, 2 \mathrm{H}$, $\mathrm{Ar}_{\text {ortho }}$ ), 7.40 - 7.34 (m, 2H, H-3, Ar ${ }_{\text {para }}$ ), $7.33-7.28$ (m, 2H, Ar $_{\text {meta }}$ ), 6.56 (dd, J = 8.4, 0.9 Hz, $1 \mathrm{H}, \mathrm{H}-4), 6.50$ (dd, $J=8.4,0.9 \mathrm{~Hz}, 1 \mathrm{H}, \mathrm{H}-2), 5.65$ (ddd, $J=17.0,10.3,8.9 \mathrm{~Hz}, 1 \mathrm{H}, \mathrm{H}-6), 5.15$ (dd, $J=10.3,1.5 \mathrm{~Hz}, 1 \mathrm{H}, \mathrm{H}-6$ ') 5.12 (ddd, $J=17.1,1.5,0.8 \mathrm{~Hz}, 1 \mathrm{H}, \mathrm{H} 6$ '), 4.90 (dd, $J=10.3$, $0.9 \mathrm{~Hz}, 1 \mathrm{H}, \mathrm{H}-5 \mathrm{a}), 3.88$ (s, 3H, $-\mathrm{OCH}_{3}$ ), 3.70 (s, 3H, C-11), 3.56 (d, J=0.7 Hz, 1H, H-7a), 2.74 - 2.63 (m, 1H, H-5), 1.37 (s, 3H, H-7'), 1.13 (d, $J=6.6$ Hz, 3H, H-9) ppm. ${ }^{13}$ C-NMR: $\left(126 \mathrm{MHz} \mathrm{CDCl}_{3}\right) \delta=189.3(\mathrm{C}-8), 172.6$ (C-10), 160.8 (C-1), 160.6 (C-4a), 139.1 (C-6), 137.3 $\left(\mathrm{Ar}_{\text {ortho }}\right), 136.6(\mathrm{C}-3), 130.0\left(\mathrm{Ar}_{\text {ipso }}\right), 129.8\left(\mathrm{Ar}_{\text {para }}\right), 129.0\left(\mathrm{Ar}_{\text {meta }}\right), 118.1(\mathrm{C}-6$ ') $113.0(\mathrm{C}-8 \mathrm{a})$, 110.6 (C-4), 104.3 (C-2), 79.9 (C-5a), $58.6(\mathrm{C}-7), 56.3\left(-\mathrm{OCH}_{3}\right), 53.6(\mathrm{C}-7 \mathrm{a}), 52.5$ (C-11), 41.4 
(C-5), 19.7 (C-7'), 17.5 (C-9) ppm. HRMS (ESI+): m/z calc. for $\mathrm{C}_{24} \mathrm{H}_{26} \mathrm{O}_{5} \mathrm{SNa}[\mathrm{M}+\mathrm{Na}]^{+}$: 449.1404, found 449.1416. FT-IR: (neat): $\tilde{v}=2975$ (w), 2944 (w), 1730 (s), 1672 (s), 1599 (s), 1575 (w), 1470 (s), 1436 (w), 1377 (w), 1327 (w), 1282 (w), 1248 (s), 1232 (w), 1171 (w), 1134 (w), 1082 (s), $1026(\mathrm{w}), 992(\mathrm{w}), 920(\mathrm{~m}), 821(\mathrm{w}), 782(\mathrm{w}), 751(\mathrm{~m}), 693(\mathrm{~m}), 537(\mathrm{~m}) \mathrm{cm}^{-1}$. $[\alpha]_{\mathrm{D}}^{20}:+126\left(\mathrm{c} 0.5, \mathrm{CHCl}_{3}\right)$.

\subsection{Methyl-2-((2R,3R)-2-((S)-but-3-en-2-yl)-5-methoxy-4-oxochroman-3-} yl)acrylate (8)

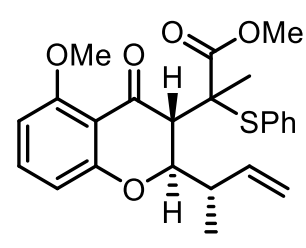

15

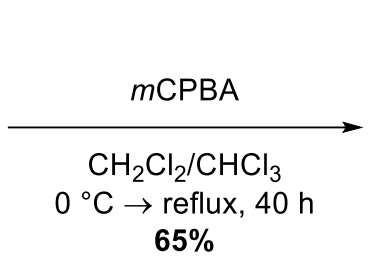

$65 \%$

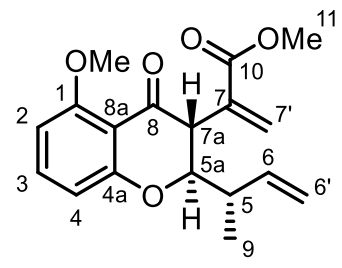

8

Sulfide 15 (mixture of diastereomers, $4.96 \mathrm{~g}, 11.6 \mathrm{mmol}$, 1.00 equiv) was dissolved in $\mathrm{CH}_{2} \mathrm{Cl}_{2}$ $(174 \mathrm{~mL})$ and the solution was cooled to $0{ }^{\circ} \mathrm{C}$. $\mathrm{mCPBA}(77 \mathrm{wt} \%, 2.87 \mathrm{~g}, 12.8 \mathrm{mmol}, 1.10$ equiv) was added and the mixture was stirred for $1 \mathrm{~h}$ at $0{ }^{\circ} \mathrm{C} . \mathrm{CHCl}_{3}(174 \mathrm{~mL})$ was added to the suspension and the now obtained solution was warmed to $65^{\circ} \mathrm{C}$ and stirred for $40 \mathrm{~h}$. Silica was added after cooling to rt, and all volatiles were removed. The crude product adsorbed on silica was purified by flash chromatography on silica (4:1 n-pentane/EtOAc) to yield diene 8 ( $2.40 \mathrm{~g}$, $7.59 \mathrm{mmol}, 65 \%$ ) as colorless solid.

TLC: $\mathrm{R}_{f}=0.22\left(3: 1 n\right.$-pentane/EtOAc). ${ }^{1} \mathbf{H}$-NMR: $\left(500 \mathrm{MHz}, \mathrm{CDCl}_{3}\right) \delta=7.37(\mathrm{t}, J=8.4 \mathrm{~Hz}$, $1 \mathrm{H}, \mathrm{H}-3), 6.57$ (dd, $J=8.3,1.0 \mathrm{~Hz}, 1 \mathrm{H}, \mathrm{H}-4), 6.50$ (dd, $J=8.5,0.9 \mathrm{~Hz}, 1 \mathrm{H}, \mathrm{H}-2), 6.46$ (d, $J=$ 0.8 Hz, 1H, H-7'), 5.99 - 5.87 (m, 1H, H-6), 5.67 (t, J=0.7 Hz, 1H, H-7'), 5.12 - 5.09 (m, 1H,

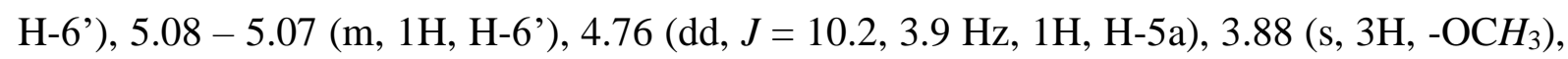
$3.78-3.75$ (m, 1H, H-7a), 3.75 (s, 3H, H-11), 2.48 - 2.39 (m, 1H, H-5), 1.12 (d, J=6.9 Hz, 3H, H-9) ppm. 13C-NMR: (126 MHz, $\mathrm{CDCl}_{3}$ ) $\delta=190.7$ (C-8), 166.3 (C-10), 162.7 (C-4a), 161.0 (C-1), 140.1 (C-6), 136.1 (C-3), 135.6 (C-7), 130.4 (C-7'), 115.7 (C-6'), 111.1 (C-8a), 110.1 (C-4), 103.9 (C-2), 82.1 (C-5a), $56.3\left(-\mathrm{OCH}_{3}\right), 54.3$ (C-7a), 52.3 (C-11), 39.4 (C-5), 13.2 (C-9) ppm. HRMS (ESI+): m/z calc. for $\mathrm{C}_{18} \mathrm{H}_{21} \mathrm{O}_{5}[\mathrm{M}+\mathrm{H}]^{+}: 317.1395$, found 317.1395 . FT-IR: (neat): $\tilde{v}=3015(\mathrm{w}), 2964(\mathrm{w}), 2931(\mathrm{w}), 2838(\mathrm{w}), 1708(\mathrm{w}), 1681(\mathrm{~s}), 1630(\mathrm{w}), 1597(\mathrm{~m})$, 1573 (w), 1468 (s), 1443 (w), 1387 (w), 1326 (m), 1280 (w), 1262 (w), 1240 (s), 1201 (m), $1170(\mathrm{~m}), 1098$ (s), 1076 (w), 1023 (w), 986 (w), 956 (m), 922 (w), 860 (w), 828 (w), 791 (m), $767(\mathrm{w}), 733(\mathrm{w}), 716(\mathrm{~m}), 664(\mathrm{w}), 647$ (w), $587(\mathrm{w}), 547(\mathrm{w}), 527(\mathrm{w}), 491(\mathrm{w}) \mathrm{cm}^{-1}$. m.p.: $85^{\circ} \mathrm{C}\left(\mathrm{Et}_{2} \mathrm{O}\right) .[\alpha]_{\mathrm{D}}^{20}:+42.7\left(\mathrm{c} 0.5, \mathrm{CHCl}_{3}\right)$. 


\subsection{Methyl 2-((2R,3S,4R)-2-((S)-but-3-en-2-yl)-4-hydroxy-5-methoxychroman-3- yl)acrylate (26b)}

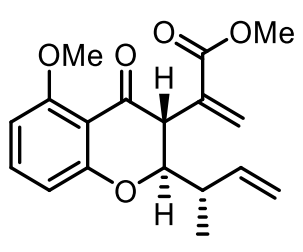

8

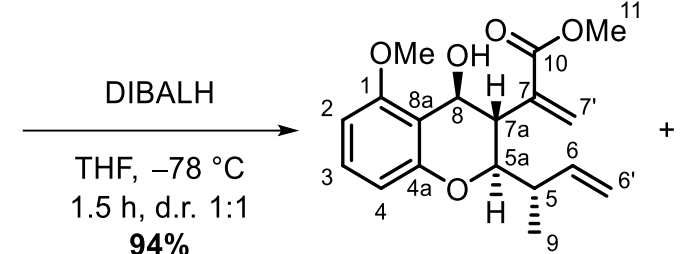

26a

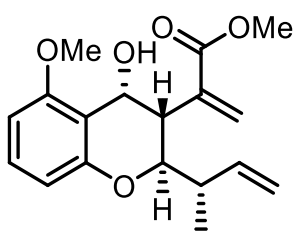

26b

Diene 8 (3.18 g, $10.1 \mathrm{mmol}, 1.00$ equiv) was dissolved in THF (50.3 mL) and the solution was cooled to $-78{ }^{\circ} \mathrm{C}$. DIBALH ( $1 \mathrm{M}$ in $\mathrm{CH}_{2} \mathrm{Cl}_{2}, 22.6 \mathrm{~mL}, 22.6 \mathrm{mmol}, 2.25$ equiv) was added dropwise and the deep red solution was stirred for $2 \mathrm{~h}$. The now pale red solution was quenched by the addition of sat. $\mathrm{Na} / \mathrm{K}$-tartrate solution $(150 \mathrm{~mL})$ and the mixture was stirred for $1 \mathrm{~h}$ at $\mathrm{rt}$. The aqueous layer was extracted with $\mathrm{CH}_{2} \mathrm{Cl}_{2}(4 \mathrm{x} 100 \mathrm{~mL})$ and the combined organic layers were dried over $\mathrm{MgSO}_{4}$, filtered and concentrated in vacuo. The crude product was purified by flash chromatography on silica (5:1 n-pentane/EtOAc) to yield a mixture of alcohol 26a and 26b (2.68 g, $8.42 \mathrm{mmol}, 84 \%)$ as colorless foam.

Note: Separation of diastereomers at this stage is possible if needed. We mostly separated the diastereomers roughly via column chromatography and used $\mathbf{2 6} \mathbf{b}$ contaminated with little amounts of $\mathbf{2 6 a}$ for the subsequent step, as separation is also possible after the Grubbsmetathesis. The spots on the TLC are no longer visible under UV-light after reduction. $\mathrm{KMnO}_{4}$ stain is the optimal choice for the TLC in this reaction.

First eluting diastereomere $\beta$-alcohol 26a (slowly crystalizing oil):

TLC: $\mathrm{R}_{f}=0.30$ (3:1 $n$-pentane/EtOAc). ${ }^{1} \mathbf{H}$-NMR: $\left(500 \mathrm{MHz}, \mathrm{CDCl}_{3}\right) \delta=7.13(\mathrm{t}, J=8.2 \mathrm{~Hz}$, 1H, H-3), 6.53 (dd, $J=8.3,0.7 \mathrm{~Hz}, 1 \mathrm{H}, \mathrm{H}-4), 6.47$ (dd, $J=8.2,0.7 \mathrm{~Hz}, 1 \mathrm{H}, \mathrm{H}-2), 6.38$ (d, $J=$ $0.9 \mathrm{~Hz}, 1 \mathrm{H}, \mathrm{H}-7^{\prime}$ '), 5.96 (ddd, $J=17.5,10.4,7.4$ Hz, 1H, H-6), 5.70 (s, 1H, H-7'), 5.12 - 5.08 (m, 1H, H-6'), 5.08 - 5.04 (m, 2H, H-6', H-8), 4.22 (dd, J = 8.0, 4.7 Hz, 1H, H-5a), 3.87 (s, $\left.3 \mathrm{H},-\mathrm{OCH}_{3}\right), 3.79$ (s, 3H, H-11), 3.62 (d, J=1.7 Hz, 1H, 8-OH), 3.22 (t, J= 7.5 Hz, 1H, H-7a), $2.61-2.53$ (m, 1H, H-5), 1.08 (d, $J=6.8 \mathrm{~Hz}, 3 \mathrm{H}, \mathrm{H}-9)$ ppm. ${ }^{13} \mathbf{C}-\mathbf{N M R}:\left(126 \mathrm{MHz}, \mathrm{CDCl}_{3}\right) \delta$ = 167.0 (C-10), 158.6 (C-1), 155.0 (C-4a), 141.5 (C-6), 139.2 (C-7), 129.2 (C-3), 128.7 (C-7'), 115.0 (C-6'), 114.0 (C-8a), 110.6 (C-4), 102.7 (C-2), 79.8 (C-5a), 66.3 (C-8), 55.8 (-OCH $)$, 52.1 (C-11), 45.4 (C-7a), 38.7 (C-5), 13.6 (C-9) ppm. HRMS (ESI+): m/z calc. for $\mathrm{C}_{18} \mathrm{H}_{22} \mathrm{O}_{5} \mathrm{Na}$ $[\mathrm{M}+\mathrm{Na}]^{+}:$341.1359, found: 341.1372. FT-IR: (neat): $\tilde{v}=3590(\mathrm{w}), 2960(\mathrm{w}), 1704(\mathrm{~m}), 1603$ (w), 1580 (m), 1475 (m), 1440 (w), 1378 (w), 1332 (m), 1296 (w), 1266 (m), 1230 (m), 1194 (w), 1124 (m), 1084 (w), 1068 (s), 1034 (w), 983 (w), 961 (w), 924 (w), $895(w), 859(w), 837$ 
(w), $786(\mathrm{w}), 770(\mathrm{~m}), 742(\mathrm{~s}), 717$ (w), 605 (w), $572(\mathrm{w}), 546(\mathrm{w}) \mathrm{cm}^{-1}$. m.p.: $98^{\circ} \mathrm{C}\left(\mathrm{CH}_{2} \mathrm{Cl}_{2}\right)$. $[\boldsymbol{\alpha}]_{\mathrm{D}}^{20}:-55.9\left(\mathrm{c} 0.5, \mathrm{CHCl}_{3}\right)$.

Second eluting diastereomere $\alpha$-alcohol $26 \mathbf{b}$ (clear oil):

TLC: $\mathrm{R}_{f}=0.24\left(3: 1 n\right.$-pentane/EtOAc). ${ }^{1} \mathbf{H}$-NMR: $\left(500 \mathrm{MHz}, \mathrm{CDCl}_{3}\right) \delta=7.15(\mathrm{t}, J=8.3 \mathrm{~Hz}$, 1H, H-2), 6.53 (d, J=8.0 Hz, 1H, H-4), 6.53 (d, J=0.7 Hz, 1H, H-7'), 6.43 (dd, J = 8.2, 0.8 Hz, 1H, H-2), 6.09 (ddd, $J=17.5,10.3,7.3 \mathrm{~Hz}, 1 \mathrm{H}, \mathrm{H}-6), 5.78$ (t, $\left.J=0.8 \mathrm{~Hz}, 1 \mathrm{H}, \mathrm{H}-7^{\prime}\right), 5.12-5.04$ (m, 2H, H-6'), 4.89 (t, $J=3.3 \mathrm{~Hz}, 1 \mathrm{H}, \mathrm{H}-8), 4.47$ (dd, $J=11.0,2.4$ Hz, 1H, H-5a), 3.84 (s, 3H, $-\mathrm{OCH}_{3}$ ), 3.79 (s, 3H, C-11), 3.19 (dd, $\left.J=11.0,3.6 \mathrm{~Hz}, 1 \mathrm{H}, \mathrm{H}-7 \mathrm{a}\right), 2.63-2.56$ (m, 1H, H-5), $2.39(\mathrm{~d}, J=3.0 \mathrm{~Hz}, 1 \mathrm{H}, 8-\mathrm{OH}), 1.00(\mathrm{~d}, J=6.9 \mathrm{~Hz}, 3 \mathrm{H}, \mathrm{H}-9)$ ppm. ${ }^{13} \mathrm{C}-\mathbf{N M R}:(126 \mathrm{MHz}$, $\left.\mathrm{CDCl}_{3}\right) \delta=167.7$ (C-10), 158.4 (C-1), 155.3 (C-4a), 142.0 (C-6), 136.9 (C-7), 129.8 (C-3), 129.6 (C-7'), 114.2 (C-6'), 113.1 (C-8a), 110.1 (C-4), 102.0 (C-2), 75.9 (C-5a), 61.1 (C-8), 55.8 $\left(-\mathrm{OCH}_{3}\right), 52.4$ (C-11), 42.0 (C-7a), 38.2 (C-5), 12.4 (C-9) ppm. HRMS (ESI+): m/z calc. for $\mathrm{C}_{18} \mathrm{H}_{22} \mathrm{O}_{5} \mathrm{Na}[\mathrm{M}+\mathrm{Na}]^{+}:$341.1359, found: 341.1361. FT-IR: (neat): $\tilde{v}=3482(\mathrm{w}), 2957(\mathrm{w})$, 2872 (w), 1692 (m), 1601 (w), 1580 (m), 1473 (m), 1438 (w), 1409 (w), 1365 (w), 1338 (w), 1279 (w), 1235 (m), 1217 (w), 1174 (w), 1137 (w), 1088 (s), 1063 (w), 1020 (w), 972 (w), 940 (w), $894(\mathrm{w}), 870(\mathrm{w}), 834(\mathrm{w}), 782(\mathrm{~m}), 766(\mathrm{w}), 738(\mathrm{w}), 710(\mathrm{~m}), 605(\mathrm{w}), 587(\mathrm{w}), 558(\mathrm{w})$, $525(\mathrm{w}), 480(\mathrm{w}), 452(\mathrm{w}) \mathrm{cm}^{-1} \cdot[\boldsymbol{\alpha}]_{\mathrm{D}}^{20}:-29.7\left(\mathrm{c} 0.5, \mathrm{CHCl}_{3}\right)$.

\subsection{Methyl (3S,3aR,9R,9aS)-9-hydroxy-8-methoxy-3-methyl-3,3a,9,9a-tetrahy-} drocyclopenta[b]chromene-1-carboxylate (6b)

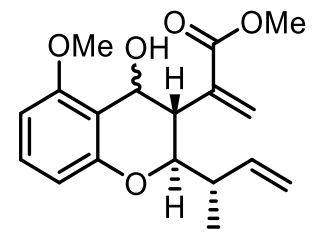

26

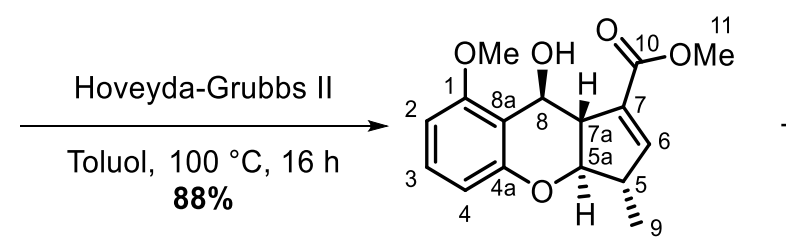

$6 a$

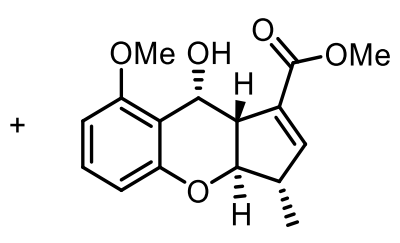

$6 \mathrm{~b}$

Alcohol 26 (300 mg, 0.94 mmol, 1.00 equiv) was dissolved in toluene (188 mL) and HoveydaGrubbs $2^{\text {nd }}$ generation catalyst $(59.0 \mathrm{mg}, 0.094 \mathrm{mmol}, 10 \mathrm{~mol} \%$ ) was added. The green solution was stirred at $100{ }^{\circ} \mathrm{C}$ for $16 \mathrm{~h}$, after which the solution turned brown. The solvent was removed in vacuo and the residue was purified by flash chromatography on silica (3:1 n-pentane/EtOAc) to yield cyclopentenes $\mathbf{6 a}(113 \mathrm{mg}, 389 \mu \mathrm{mol}, 41 \%)$, $6 \mathbf{b}(93 \mathrm{mg}, 320 \mu \mathrm{mol}, 34 \%)$ and a mixture of $\mathbf{6 a} / \mathbf{6 b}$ (36.0 mg, $124 \mu \mathrm{mol}, 13 \%)$.

First eluting diastereomere $\alpha$-alcohol $\mathbf{6 b}$ (pale brown solid):

TLC: $\mathrm{R}_{f}=0.18$ (3:1 $n$-pentane/EtOAc). ${ }^{1} \mathbf{H}-\mathbf{N M R}:\left(500 \mathrm{MHz}, \mathrm{CDCl}_{3}\right) \delta=7.19(\mathrm{t}, J=8.3 \mathrm{~Hz}$, $1 \mathrm{H}, \mathrm{H}-3), 6.69$ (dd, $J=2.4,2.0 \mathrm{~Hz}, 1 \mathrm{H}, \mathrm{H}-6), 6.59$ (d, $J=8.1 \mathrm{~Hz}, 1 \mathrm{H}, \mathrm{H}-4), 6.52$ (d, $J=8.1 \mathrm{~Hz}$, 1H, H-2), 5.50 (d, $J=3.6 \mathrm{~Hz}, 1 \mathrm{H}, \mathrm{H}-8), 3.96$ (dd, $J=10.6,9.3 \mathrm{~Hz}, 1 \mathrm{H}, \mathrm{H}-5 \mathrm{a}), 3.88$ (s, 3H, - 
$\mathrm{OCH}_{3}$ ), 3.81 (s, 3H, C-11), $3.00-2.92$ (m, 1H, C-5), 2.81 (ddt, $J=10.6,3.5,2.9 \mathrm{~Hz}, 1 \mathrm{H}, \mathrm{C}-$ 7a), 2.56 (br s, 1H, 8-OH), 1.36 (d, $J=7.0 \mathrm{~Hz}, 3 \mathrm{H}, \mathrm{C}-9)$ ppm. ${ }^{13} \mathbf{C}-\mathbf{N M R}$ : (126 MHz, CDCl ) $\delta=165.5$ (C-10), 159.5 (C-1), 156.7 (C-4a), 148.1 (C-6), 132.0 (C-7), 129.7 (C-3), 114.0 (C8a), 110.3 (C-4), 102.9 (C-2), 81.4 (C-5a), 60.7 (C-8), $55.8\left(-\mathrm{OCH}_{3}\right), 51.9$ (C-11), 49.5 (C-7a), 42.2 (C-5), 16.1 (C-9) ppm. HRMS (ESI+): m/z calc. for $\mathrm{C}_{16} \mathrm{H}_{18} \mathrm{O}_{5} \mathrm{Na}[\mathrm{M}+\mathrm{Na}]^{+}:$313.1046, found: 313.1052. FT-IR: (neat): $\tilde{v}=3590$ (w), 2960 (w), 1704 (m), 1603 (w), 1580 (m), 1475 (m), 1440 (w), 1378 (w), 1332 (m), 1296 (w), 1266 (m), 1230 (m), 1194 (w), 1124 (m), 1084 (w), $1068(\mathrm{~s}), 1034(\mathrm{w}), 983(\mathrm{w}), 961(\mathrm{w}), 924(\mathrm{w}), 895(\mathrm{w}), 859(\mathrm{w}), 837(\mathrm{w}), 786(\mathrm{w}), 770$

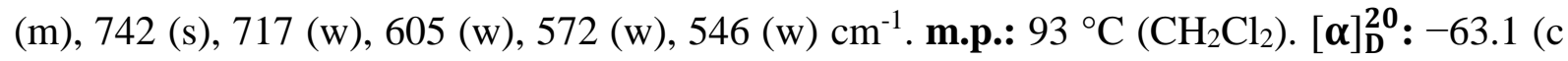
$\left.0.5, \mathrm{CHCl}_{3}\right)$.

Second eluting diastereomere $\beta$-alcohol 6a (brown oil):

TLC: $\mathrm{R}_{f}=0.12\left(3: 1 n\right.$-pentane/EtOAc). ${ }^{1} \mathbf{H}$-NMR: $\left(500 \mathrm{MHz}, \mathrm{CDCl}_{3}\right) \delta=7.14(\mathrm{t}, J=8.2 \mathrm{~Hz}$, 1H, H-3), $6.66-6.65$ (m, 1H, H-6), 6.55 (d, J=8.3 Hz, 1H, H-4), 6.54 (d, J = 8.2 Hz, 1H, H2), $5.23(\mathrm{~d}, J=9.7 \mathrm{~Hz}, 1 \mathrm{H}, \mathrm{H}-8), 4.61(\mathrm{~d}, J=1.0 \mathrm{~Hz}, 1 \mathrm{H}, 8-\mathrm{OH}), 3.89$ (s, 3H, $\left.-\mathrm{OCH}_{3}\right), 3.81$ (s, 3H, C-11), 3.56 (dd, $J=10.6,9.3 \mathrm{~Hz}, 1 \mathrm{H}, \mathrm{H}-5 \mathrm{a}), 3.08$ (ddt, $J=10.5,9.9,2.7 \mathrm{~Hz}, 1 \mathrm{H}, \mathrm{H}-7 \mathrm{a}$ ), $3.02-2.94$ (m, 1H, H-5), 1.29 (d, J=7.0 Hz, 3H, H-9) ppm. ${ }^{13} \mathbf{C}-\mathbf{N M R}:\left(126 \mathrm{MHz}, \mathrm{CDCl}_{3}\right) \delta$ = 165.8 (C-10), 160.1 (C-1), 157.6 (C-4a), 147.9 (C-6), 134.2 (C-7), 129.2 (C-3), 115.2 (C-8a), 110.4 (C-4), 104.4 (C-2), 85.9 (C-5a), 67.4 (C-8), 56.1 (-OCH $), 52.1$ (C-11), 52.0 (C-7a), 42.1 (C-5), 15.9 (C-9) ppm. HRMS (ESI+): m/z calc. for $\mathrm{C}_{16} \mathrm{H}_{18} \mathrm{O}_{5} \mathrm{Na}[\mathrm{M}+\mathrm{Na}]^{+}:$313.1046, found: 313.1053. FT-IR: (neat): $\tilde{v}=3482$ (w), 2957 (w), 2872 (w), 1692 (m), 1601 (w), 1580 (m), 1473 (m), 1438 (w), 1409 (w), 1365 (w), 1338 (w), 1279 (w), 1235 (m), 1217 (w), 1174 (w), $1137(w), 1088$ (s), 1063 (w), 1020 (w), 972 (w), 940 (w), 894 (w), 870 (w), 834 (w), 782 (m), $766(\mathrm{w}), 738(\mathrm{w}), 710(\mathrm{~m}), 605(\mathrm{w}), 587(\mathrm{w}), 558(\mathrm{w}), 525(\mathrm{w}), 480(\mathrm{w}), 452(\mathrm{w}) \mathrm{cm}^{-1} \cdot[\boldsymbol{\alpha}]_{\mathrm{D}}^{20}$ : $+63.1\left(\mathrm{c} 0.5, \mathrm{CHCl}_{3}\right)$.

\subsection{Methyl (1R,2S,3S,3aR,9S,9aS)-1,2,9-trihydroxy-8-methoxy-3-methyl-1,2,3,3a,9,9a-} hexahydrocyclopenta[b]chromene-1-carboxylate (17b)

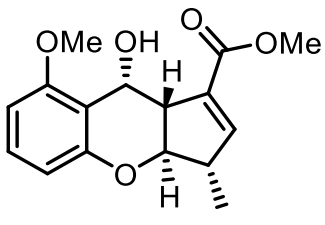

6b

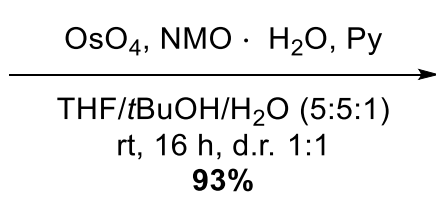

$93 \%$

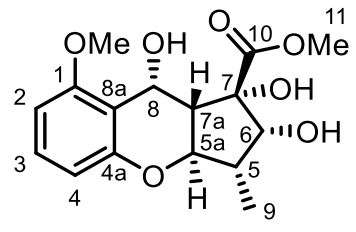

$17 \mathrm{a}$

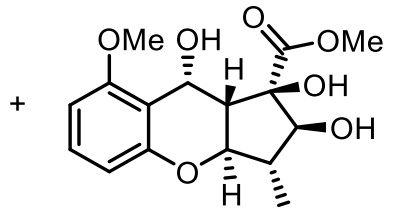

$17 \mathrm{~b}$

Cyclopentene 6b (200 mg, 0.69 mmol, 1.00 equiv) was dissolved in THF/ $t \mathrm{BuOH} / \mathrm{H}_{2} \mathrm{O}$ (5:5:1). Pyridine (132 $\mu \mathrm{L}, 1.63 \mathrm{mmol}, 1.70$ equiv), $\mathrm{OsO}_{4}(2.5 \mathrm{wt} \%$ in $t \mathrm{BuOH}, 1.73 \mathrm{~mL}, 0.14 \mathrm{mmol}$, $20 \mathrm{~mol} \%)$ and $\mathrm{NMO} \cdot \mathrm{H}_{2} \mathrm{O}(675 \mathrm{mg}, 5.76 \mathrm{mmol}, 6.00$ equiv) were added successively. The 
resulting brown solution was stirred for $24 \mathrm{~h}$ at $\mathrm{rt}$, after which it turned yellow. The reaction was quenched with aq. sat. $\mathrm{Na}_{2} \mathrm{~S}_{2} \mathrm{O}_{3}$ and stirred for $30 \mathrm{~min}$. EtOAc was added and the layers were separated. The aqueous layer was extracted with EtOAc $(3 \times 50 \mathrm{~mL})$ and the combined organic layers were washed with brine, dried over $\mathrm{MgSO}_{4}$, filtered and concentrated in vacuo. The crude product was purified by flash chromatography on silica (1:1 n-pentane/EtOAc) to yield triols 17a (103 mg, $318 \mu \mathrm{mol}, 46 \%)$ and 17b (106 mg, $327 \mu \mathrm{mol}, 47 \%)$.

First eluting diastereomere $\alpha$-diol 17a (off-white foam):

TLC: $\mathrm{R}_{f}=0.17\left(1: 1 n\right.$-pentane/EtOAc). ${ }^{1}$ H-NMR: $\left(500 \mathrm{MHz}\right.$, DMSO- $\left.d_{6}\right) \delta=7.16(\mathrm{t}, J=$ $8.3 \mathrm{~Hz}, 1 \mathrm{H}, \mathrm{H}-3), 6.54$ (d, $J=7.8 \mathrm{~Hz}, 1 \mathrm{H}, \mathrm{H}-2), 6.46$ (d, $J=8.2 \mathrm{~Hz}, 1 \mathrm{H}, \mathrm{H}-4), 5.84$ (s, 1H, 7-OH), $5.75(\mathrm{~d}, J=3.7 \mathrm{~Hz}, 1 \mathrm{H}, 8-\mathrm{OH}), 5.02$ (t, $J=3.4 \mathrm{~Hz}, 1 \mathrm{H}, \mathrm{H}-8), 4.85$ (d, $J=8.6 \mathrm{~Hz}, 1 \mathrm{H}$, 6-OH), 4.34 (t, $J=9.1 \mathrm{~Hz}, 1 \mathrm{H}, \mathrm{H}-6), 4.22$ (dd, $J=11.8,8.0 \mathrm{~Hz}, 1 \mathrm{H}, \mathrm{H}-5 \mathrm{a}), 3.76$ (s, 3H, -OCH ), $^{-}$ 3.71 (s, 3H, H-11), 2.31 (dp, $J=9.3,7.2 \mathrm{~Hz}, 1 \mathrm{H}, \mathrm{H}-5), 2.03$ (dd, $J=11.9,3.1 \mathrm{~Hz}, 1 \mathrm{H}, \mathrm{H}-7 \mathrm{a})$, 1.10 (d, $J=7.2 \mathrm{~Hz}, 3 \mathrm{H}, \mathrm{H}-9)$ ppm. ${ }^{13} \mathrm{C}-\mathrm{NMR}$ : (126 MHz, DMSO-d $\left.d\right) \delta=173.8(\mathrm{C}-10), 158.4$ (C-1), 155.2 (C-4a), 129.8 (C-3), 113.7 (C-8a), 109.5 (C-4), 102.7 (C-2), 81.1 (C-7), 78.8 (C5a), 75.1 (C-6), 59.5 (C-8), 55.5 (-OCH 3 ), 52.2 (C-11), 46.3 (C-7a), 39.5 (C-5), 13.6 (C-9) ppm. HRMS (ESI+): $\mathrm{m} / \mathrm{z}$ calc. for $\mathrm{C}_{16} \mathrm{H}_{20} \mathrm{O}_{7} \mathrm{Na}[\mathrm{M}+\mathrm{Na}]^{+}:$347.1101, found: 347.1135. FT-IR: (neat): $\tilde{v}=3395(\mathrm{w}), 2932(\mathrm{w}), 1730(\mathrm{~m}), 1606$ (w), $1583(\mathrm{~m}), 1473(\mathrm{~m}), 1438(\mathrm{w}), 1378(\mathrm{w})$, 1332 (w), 1268 (w), 1234 (m), 1183 (w), 1120 (m), 1078 (s), 1013 (w), 953 (m), 831 (w), 778 (m), $744(\mathrm{w}), 727(\mathrm{w}), 540(\mathrm{w}) \mathrm{cm}^{-1} \cdot[\boldsymbol{\alpha}]_{\mathrm{D}}^{20}:+92.3\left(\mathrm{c} 0.5, \mathrm{CHCl}_{3}\right)$.

Second eluting diastereomere $\beta$-diol 17b (slightly brown oil):

TLC: $\mathrm{R}_{f}=0.24$ (3:1 $n$-pentane/EtOAc). ${ }^{1} \mathbf{H}-\mathbf{N M R}:\left(500 \mathrm{MHz}, \mathrm{DMSO}-d_{6}\right) \delta=7.11$ (t, $J=8.2$ Hz, 1H, H-3), 6.52 (d, J=8.2 Hz, 1H, H-2), 6.41 (d, J= 7.9 Hz, 1H, H-4), 5.30 (s, 1H, 7-OH), $5.00(\mathrm{~d}, J=7.1 \mathrm{~Hz}, 1 \mathrm{H}, 6-\mathrm{OH}), 4.89(\mathrm{dd}, J=6.3,3.0 \mathrm{~Hz}, 1 \mathrm{H}, \mathrm{H}-8), 4.83(\mathrm{~d}, J=6.0 \mathrm{~Hz}, 1 \mathrm{H}, 8-$ $\mathrm{OH}$ ), $3.84-3.79$ (m, 2H, H-6, H-5a), 3.77 (s, 3H, -OCH $), 3.59$ (s, 3H, C-11), 1.99 (dd, $J=$ 11.9, 2.9 Hz, 1H, H-7a), 1.97 - 1.89 (m, 1H, H-5), 1.22 (d, J=6.6 Hz, 3H, H-9) ppm. ${ }^{13}$ CNMR: (126 MHz, DMSO- $\left.d_{6}\right) \delta=173.8$ (C-10), 158.4 (C-1), 155.0 (C-4a), 129.2 (C-3), 114.7 (C-8a), 109.3 (C-4), 102.7 (C-2), 78.0 (C-6), 76.0 (C-7), 73.5 (C-5a), 57.2 (C-8), 56.0 (C-7a), $55.5\left(-\mathrm{OCH}_{3}\right), 51.8$ (C-11), 44.3 (C-5), 15.4 (C-9) ppm. HRMS (ESI+): m/z calc. for $\mathrm{C}_{16} \mathrm{H}_{20} \mathrm{O}_{7} \mathrm{Na}[\mathrm{M}+\mathrm{Na}]^{+}:$347.1101, found: 347.1114. FT-IR: (neat): $\tilde{v}=3427(\mathrm{w}), 2957(\mathrm{w})$, 2926 (w), 2851 (w), 1726 (m), 1606 (w), 1583 (m), 1473 (m), 1439 (w), 1379 (w), 1335 (w), 1265 (w), 1232 (s), 1177 (w), 1120 (w), 1073 (s), 1042 (w), 953 (w), 929 (w), 832 (w), 780 (m), $742(\mathrm{~m}), 705(\mathrm{~m}), 582(\mathrm{w}), 545(\mathrm{w}), 507(\mathrm{w}), 455(\mathrm{w}) \mathrm{cm}^{-1} \cdot[\boldsymbol{\alpha}]_{\mathrm{D}}^{\mathbf{2 0}}:+84.6\left(\mathrm{c} 0.5, \mathrm{CHCl}_{3}\right)$. 
2.9 Methyl (1S,2R,3S,3aR,9S,9aS)-1,2,9-trihydroxy-8-methoxy-3-methyl-1,2,3,3a,9,9ahexahydrocyclopenta[b]chromene-1-carboxylate (16)

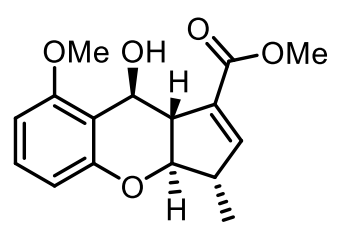

$6 a$

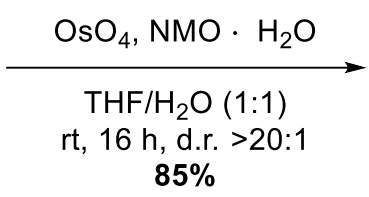

$85 \%$

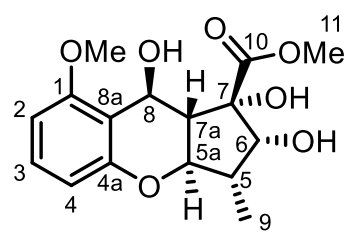

16

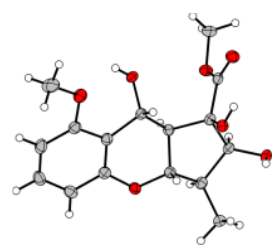

ent-16

Cyclopentene 6a (79 mg, $272 \mu \mathrm{mol}, 1.00$ equiv), $\mathrm{OsO}_{4}(2.5 \mathrm{wt} \%$ in $t \mathrm{BuOH}, 171 \mu \mathrm{L}, 13.6 \mu \mathrm{mol}$, $5 \mathrm{~mol} \%)$ and $\mathrm{NMO} \cdot \mathrm{H}_{2} \mathrm{O}\left(63.3 \mathrm{mg}, 540 \mu \mathrm{mol}, 2.00\right.$ equiv) were dissolved in $\mathrm{THF} / \mathrm{H}_{2} \mathrm{O}(1: 1$, $5.4 \mathrm{~mL}$ ) and stirred for $16 \mathrm{~h}$ at rt. The reaction was quenched by addition of sat. aq. $\mathrm{Na}_{2} \mathrm{~S}_{2} \mathrm{O}_{3}$ and stirred $30 \mathrm{~min}$. EtOAc was added and the layers were separated. The aqueous layer was extracted with EtOAc (3x $20 \mathrm{~mL})$ and the combined organic layers were washed with brine, dried over $\mathrm{MgSO}_{4}$, filtered and concentrated in vacuo. The crude product was purified by flash chromatography on silica (1:1 n-pentane/EtOAc) to yield triol $16(75 \mathrm{mg}, 231 \mu \mathrm{mol}, 85 \%)$ as colorless solid. A suitable crystal of ent-16 for structure determination was obtained by cooling a concentrated $\mathrm{Et}_{2} \mathrm{O}$ solution in a small vial standing in a bigger vial filled with $n$-pentane to $-20{ }^{\circ} \mathrm{C}$ for 2 days.

TLC: $\mathrm{R}_{f}=0.17\left(1: 1 n\right.$-pentane/EtOAc). ${ }^{1} \mathbf{H}$-NMR: $\left(500 \mathrm{MHz}\right.$, DMSO-d $\left.d_{6}\right) \delta=7.09(\mathrm{t}, J=$ $8.2 \mathrm{~Hz}, 1 \mathrm{H}, \mathrm{H}-3), 6.54$ (dd, $J=8.3,0.9 \mathrm{~Hz}, 1 \mathrm{H}, \mathrm{H}-2), 6.43$ (dd, $J=8.3,0.9 \mathrm{~Hz}, 1 \mathrm{H}, \mathrm{H}-4), 5.22$ (d, $J=7.7 \mathrm{~Hz}, 1 \mathrm{H}, 6-\mathrm{OH}), 5.07$ (dd, $J=9.8,3.7 \mathrm{~Hz}, 1 \mathrm{H}, \mathrm{H}-8), 4.95$ (d, $J=1.0 \mathrm{~Hz}, 1 \mathrm{H}, 7-\mathrm{OH})$, $4.49(\mathrm{~d}, J=3.7 \mathrm{~Hz}, 1 \mathrm{H}, 8-\mathrm{OH}), 4.40$ (dd, $J=9.7,7.7 \mathrm{~Hz}, 1 \mathrm{H}, \mathrm{H}-6), 3.76$ (s, $J=3.9 \mathrm{~Hz}, 3 \mathrm{H}$, $\left.\mathrm{OCH}_{3}\right), 3.65$ (dd, $\left.J=13.1,7.0 \mathrm{~Hz}, 1 \mathrm{H}, \mathrm{H}-5 \mathrm{a}\right), 3.65$ (s, 3H, H-11), $2.35-2.25$ (m, 2H, H-7a, H5), 1.01 (d, $J=7.2 \mathrm{~Hz}, 3 \mathrm{H}, \mathrm{H}-9)$ ppm. ${ }^{13} \mathrm{C}-\mathrm{NMR}$ : (126 MHz, DMSO-d $) \delta=174.4$ (C-10), 159.3 (C-1), 156.0 (C-4a), 128.6 (C-3), 116.4 (C-8a), 109.3 (C-4), 103.5 (C-2), 83.9 (C-5a), 78.2 (C-7), 74.8 (C-6), 61.3 (C-8), 55.4 (-OCH $\left.{ }_{3}\right), 52.0$ (C-7a), 51.8 (C-11), 39.4 (C-5), 13.0 (C9) ppm. HRMS (ESI+): m/z calc. for $\mathrm{C}_{16} \mathrm{H}_{20} \mathrm{O}_{7} \mathrm{Na}[\mathrm{M}+\mathrm{Na}]^{+}:$347.1101, found: 347.1116. FTIR: (neat): $\tilde{v}=3416$ (w), 2950 (w), 1727 (m), 1604 (w), 1579 (m), 1470 (m), 1437 (w), 1380 (w), $1326(\mathrm{w}), 1298$ (w), 1257 (w), 1231 (m), 1198 (w), 1183 (w), 1148 (w), 1115 (w), 1083 (s), $1019(\mathrm{~m}), 979(\mathrm{w}), 958(\mathrm{w}), 940(\mathrm{w}), 817(\mathrm{w}), 776(\mathrm{~m}), 741(\mathrm{w}), 720(\mathrm{w}), 626(\mathrm{w}), 605(\mathrm{w})$, $545(\mathrm{w}), 519(\mathrm{w}), 458(\mathrm{~m}) \mathrm{cm}^{-1}$. m.p.: $118{ }^{\circ} \mathrm{C}\left(\mathrm{Et}_{2} \mathrm{O}\right) .[\boldsymbol{\alpha}]_{\mathrm{D}}^{\mathbf{2 0}}:+95.6\left(\mathrm{c} 0.5, \mathrm{CHCl}_{3}\right)$. 


\section{1,2,3,3a,9,9a-hexahydrocyclopenta[b]chromene-1-carboxylate (18a)}

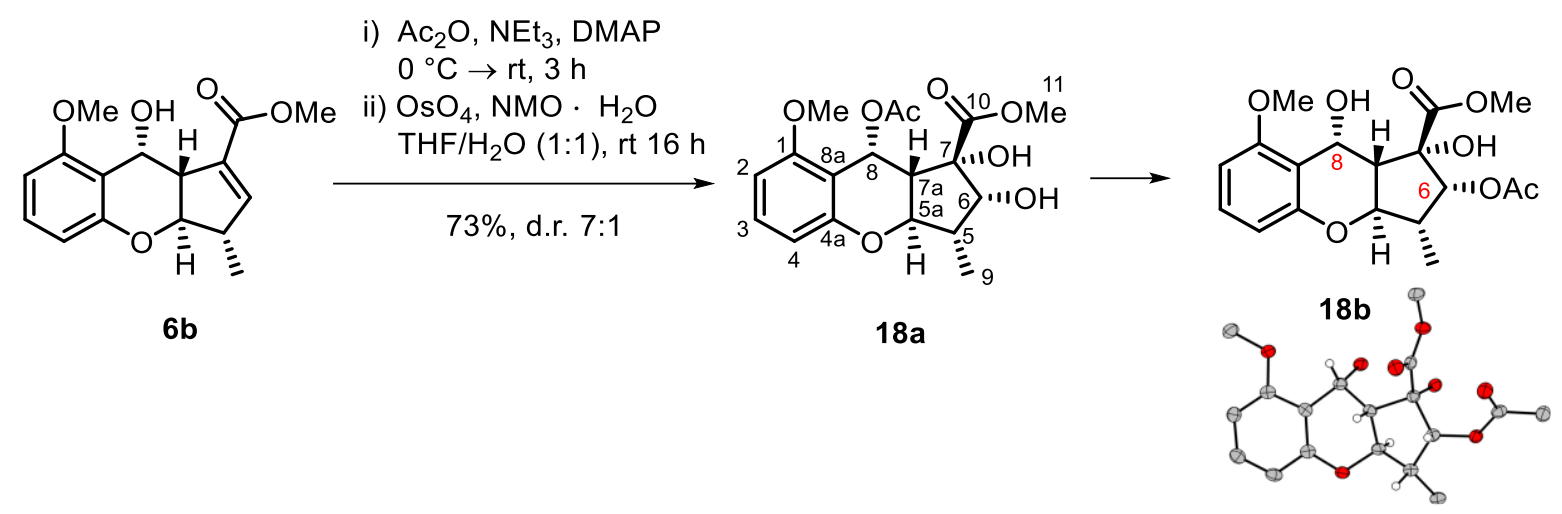

Cyclopentene 6b (118 mg, $0.41 \mathrm{mmol}, 1.00$ equiv) and DMAP (5.0 mg, $0.04 \mathrm{mmol}, 10 \mathrm{~mol} \%$ ) were suspended in $\mathrm{NEt}_{3}\left(845 \mu \mathrm{L}, 6.10 \mathrm{mmol}, 15.0\right.$ equiv) and cooled to $0{ }^{\circ} \mathrm{C}$. $\mathrm{Ac}_{2} \mathrm{O}(231 \mu \mathrm{L}$, $2.44 \mathrm{mmol}, 6.00$ equiv) was added and the mixture was warmed to rt. After $3 \mathrm{~h}$ stirring at $\mathrm{rt}$, the mixture was cooled to $0{ }^{\circ} \mathrm{C}$ and $\mathrm{MeOH}(2 \mathrm{~mL})$ was added. The solution was again warmed to $\mathrm{rt}$ and all volatiles were removed in vacuo. The residue was dissolved in $\mathrm{CH}_{2} \mathrm{Cl}_{2}(20 \mathrm{~mL})$ and $\mathrm{H}_{2} \mathrm{O}(20 \mathrm{~mL})$ was added. The layers were separated and the aqueous layers was extruded with $\mathrm{CH}_{2} \mathrm{Cl}_{2}$ (3x $20 \mathrm{~mL}$ ). The combined organic layers were dried over $\mathrm{MgSO}_{4}$, filtered and concentrated in vacuo. The obtained colorless foam was dissolved in $\mathrm{THF} / \mathrm{H}_{2} \mathrm{O}(1: 1,8.2 \mathrm{~mL})$ and $\mathrm{OsO}_{4}(2.5 \mathrm{wt} \%$ in $t \mathrm{BuOH}, 255 \mu \mathrm{L}, 20.4 \mu \mathrm{mol}, 5 \mathrm{~mol} \%)$ and $\mathrm{NMO} \cdot \mathrm{H}_{2} \mathrm{O}(96.0 \mathrm{mg}$, $0.82 \mathrm{mmol}, 2.00$ equiv) were added successively. After stirring for $16 \mathrm{~h}$ at $\mathrm{rt}$, the reaction as quenched by addition of aq. sat. $\mathrm{Na}_{2} \mathrm{~S}_{2} \mathrm{O}_{3}$ and the mixture was stirred for $30 \mathrm{~min}$. EtOAc was added and the layers were separated. The aqueous layer was extracted with EtOAc $(3 \times 20 \mathrm{~mL})$ and the combined organic layers were washed with brine, dried over $\mathrm{MgSO}_{4}$, filtered and concentrated in vacuo. The crude product was purified by flash chromatography on silica (1:2 $n$-pentane/EtOAc) to yield acetate $\mathbf{1 8}(109 \mathrm{mg}, 298 \mu \mathrm{mol}, 73 \%)$ as colorless powder.

Note: ${ }^{1} H$-NMR spectra after column chromatography clearly shows the acetate to be on the 8-OH. Anyways, after slow evaporation (about 4 weeks) of a solution of 18 a in Et $t_{2} \mathrm{O} / \mathrm{n}$-pentane ( 2:1) in an NMR-tube, an X-ray suitable crystal could be obtained which showed the acetate has switched to the 6-OH. Presumably, a series of intramolecular 1,2-acyl shifts to the most accessible OH-group lead to acetate $\mathbf{1 8 b}$.

Analytical data for 18a:

TLC: $\mathrm{R}_{f}=0.19\left(1: 1 n\right.$-pentane/EtOAc). ${ }^{1} \mathbf{H}$-NMR: $\left(500 \mathrm{MHz}, \mathrm{CDCl}_{3}\right) \delta=7.20(\mathrm{t}, J=8.3 \mathrm{~Hz}$, $1 \mathrm{H}, \mathrm{H}-3), 6.54$ (dd, $J=8.3,1.0 \mathrm{~Hz}, 1 \mathrm{H}, \mathrm{H}-4), 6.45$ (dd, $J=8.2,1.0 \mathrm{~Hz}, 1 \mathrm{H}, \mathrm{H}-2), 6.26$ (d, $J=$ $3.2 \mathrm{~Hz}, 1 \mathrm{H}, \mathrm{H}-8), 4.33$ (t, $J=10.1 \mathrm{~Hz}, 1 \mathrm{H}, \mathrm{H}-6), 4.20$ (dd, $J=12.2,7.3 \mathrm{~Hz}, 1 \mathrm{H}, \mathrm{H}-5 \mathrm{a}), 3.88$ (s, $3 \mathrm{H}, \mathrm{H}-11), 3.78$ (s, 3H, -OCH $), 3.50$ (s, 1H, 7-OH), 2.54 (d, J = 10.2 Hz, 1H, 6-OH), 2.48 (dd, 
$J=12.2,3.2 \mathrm{~Hz}, 1 \mathrm{H}, \mathrm{H}-7 \mathrm{a}), 2.45$ (dq, $J=10.0,7.3 \mathrm{~Hz}, 1 \mathrm{H}, \mathrm{H}-5), 2.02$ (s, 3H, $\left.H_{3} \mathrm{CC}(\mathrm{O}) \mathrm{O}-\right)$, 1.22 (d, $J=7.3 \mathrm{~Hz}, 3 \mathrm{H}, \mathrm{H}-9)$ ppm. ${ }^{13} \mathrm{C}-\mathbf{N M R}:\left(126 \mathrm{MHz}, \mathrm{CDCl}_{3}\right) \delta=173.7(\mathrm{C}-10), 171.1$ ( $\left.\mathrm{H}_{3} \mathrm{CC}(\mathrm{O}) \mathrm{O}-\right), 158.9$ (C-1), 156.4 (C-4a), 130.7 (C-3), 110.2 (C-8a), 110.1 (C-4), 102.6 (C-2), 80.2 (C-7), 79.9 (C-5a), 76.1 (C-6), 60.7 (C-8), 55.9 (-OCH $), 53.6$ (C-11), 45.7 (C-7a), 39.7 (C-5), $21.4\left(\mathrm{H}_{3} \mathrm{CC}(\mathrm{O}) \mathrm{O}-\right), 13.4$ (C-9) ppm. HRMS (ESI+): m/z calc. for $\mathrm{C}_{18} \mathrm{H}_{22} \mathrm{O}_{8} \mathrm{Na}[\mathrm{M}+\mathrm{Na}]^{+}$: 389.1207, found: 389.1213. FT-IR: (neat): $\tilde{v}=3433(\mathrm{w}), 2933$ (w), 1732 (m), 1607 (w), 1585 (w), 1472 (w), 1439 (w), 1371 (m), 1335 (m), 1271 (m), 1235 (m), 1188 (m), 1169 (m), 1137 (m), 1080 (m), 1016 (m), 949 (m), 859 (s), 823 (s), 779 (s), 727 (s), 705 (s), 685 (s), 613 (s),

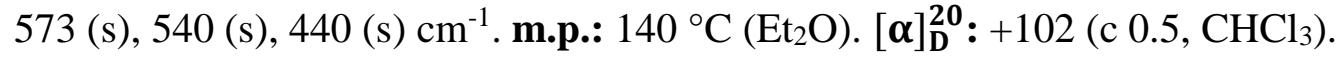

2.11 Methyl (1R,3R,3aS,9R,9aS)-1,9-dihydroxy-8-methoxy-3-methyl-2-oxo-1,2,3,3a,9,9ahexahydrocyclopenta[b]chromene-1-carboxylate (19)

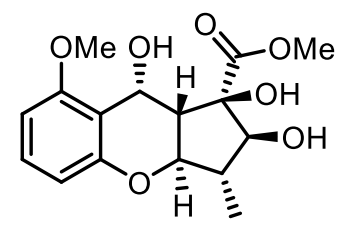

$17 \mathrm{~b}$

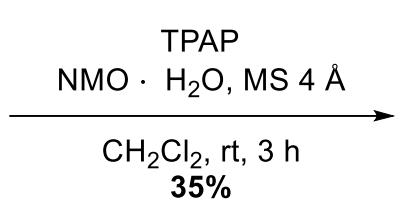

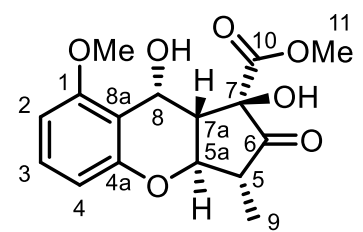

19

Triol 17b (68.0 mg, $210 \mu \mathrm{mol}, 1.00$ equiv) was dissolved in $\mathrm{CH}_{2} \mathrm{Cl}_{2}(6.0 \mathrm{~mL})$ and $\mathrm{MS} 4 \AA$ (200 mg), NMO $\cdot \mathrm{H}_{2} \mathrm{O}(73.7 \mathrm{mg}, 629 \mu \mathrm{mol}, 3.00$ equiv) and TPAP (7.4 mg, $21.0 \mu \mathrm{mol}$, $10 \mathrm{~mol} \%$ ) were added successively. The dark green reaction mixture was stirred for $3 \mathrm{~h}$, after which it turned deep brown. The mixture was directly loaded on a silica column and eluted with 2:1 $n$-pentane/EtOAc. Ketone 19 (24 mg, $74.5 \mu \mathrm{mol}, 35 \%)$ was isolated as colorless powder. TLC: $\mathrm{R}_{f}=0.33\left(1: 1 n\right.$-pentane/EtOAc). ${ }^{1} \mathbf{H}$-NMR: $\left(500 \mathrm{MHz}, \mathrm{CDCl}_{3}\right) \delta=7.20(\mathrm{t}, J=8.3 \mathrm{~Hz}$, $1 \mathrm{H}, \mathrm{H}-3), 6.59$ (dd, $J=8.4,1.0 \mathrm{~Hz}, 1 \mathrm{H}, \mathrm{H}-4), 6.51$ (dd, $J=8.2,0.9 \mathrm{~Hz}, 1 \mathrm{H}, \mathrm{H}-2), 5.21$ (d, $J=$ $4.0 \mathrm{~Hz}, 1 \mathrm{H}, \mathrm{H}-8), 4.49$ (dd, $J=11.6,8.8 \mathrm{~Hz}, 1 \mathrm{H}, \mathrm{H}-5 \mathrm{a}), 3.86$ (s, 3H, -OCH $), 3.74$ (s, 3H, H-11), $2.70(\mathrm{dq}, J=8.8,7.1 \mathrm{~Hz}, 1 \mathrm{H}, \mathrm{H}-5), 2.49$ (dd, $J=11.6,4.0 \mathrm{~Hz}, 1 \mathrm{H}, \mathrm{H}-7 \mathrm{a}), 1.45$ (d, $J=7.1 \mathrm{~Hz}$, 3H, H-9) ppm. ${ }^{13} \mathrm{C}-\mathrm{NMR}:\left(126 \mathrm{MHz}, \mathrm{CDCl}_{3}\right) \delta=211.1$ (C-6), 171.8 (C-10), 158.9 (C-1), 154.9 (C-4a), 130.2 (C-3), 114.2 (C-8a), 110.6 (C-4), 103.0 (C-2), 80.3 (C-7), 73.9 (C-5a), 58.2 (C8), $55.8\left(-\mathrm{OCH}_{3}\right), 53.5$ (C-11), 52.2 (C-7a), 48.8 (C-5), 13.5 (C-9) ppm. HRMS (ESI+): m/z calc. for $\mathrm{C}_{16} \mathrm{H}_{18} \mathrm{O}_{7} \mathrm{Na}[\mathrm{M}+\mathrm{Na}]^{+}: 345.0945$, found: 345.0952 . FT-IR: (neat): $\tilde{v}=3379(\mathrm{~m}), 2923$ (s), 2847 (w), 1766 (w), 1747 (s), 1603 (w), 1582 (s), 1461 (s), 1439 (w), 1387 (m), 1376 (w), $1276(\mathrm{~m}), 1232$ (m), 1200 (w), 1174 (w), 1150 (w), 1111 (w), 1070 (s), 1042 (w), 990 (m), 971 (w), $956(\mathrm{w}), 921(\mathrm{~m}), 908(\mathrm{w}), 840(\mathrm{~s}), 779(\mathrm{~s}), 744(\mathrm{~s}), 708(\mathrm{~m}), 549(\mathrm{~m}), 515(\mathrm{~s}), 468(\mathrm{~s}) \mathrm{cm}^{-1}$. m.p.: $200{ }^{\circ} \mathrm{C}(\mathrm{EtOAc}) .[\boldsymbol{\alpha}]_{\mathrm{D}}^{\mathbf{2 0}}:+47.4\left(\mathrm{c} 0.5, \mathrm{CHCl}_{3}\right)$. 


\subsection{Methyl $\quad(1 R, 3 R, 3 \mathrm{a} R, 9 \mathrm{a} S)-1,8-d i h y d r o x y-3-m e t h y l-2,9-d i o x o-1,2,3,3 a, 9,9 a-$}

hexahydrocyclopenta[b]chromene-1-carboxylate (4)

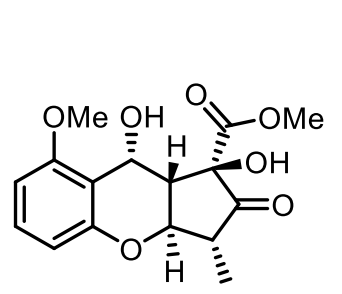

19

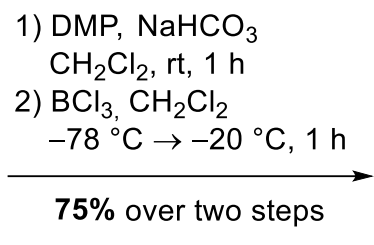

$75 \%$ over two steps

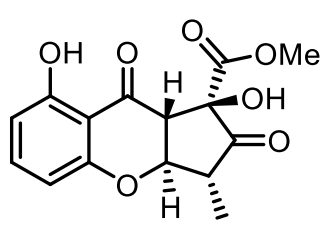

4

Ketone 19 ( $8 \mathrm{mg}, 24.8 \mu \mathrm{mol}, 1.00$ equiv) was dissolved in $\mathrm{CH}_{2} \mathrm{Cl}_{2}(2.0 \mathrm{~mL})$ and $\mathrm{NaHCO}_{3}$ (16.7 mg, $198 \mu \mathrm{mol}, 8.00$ equiv) and DMP (26.3 mg, $62.1 \mu \mathrm{mol}, 2.50$ equiv) were added successively. The mixture was stirred for $1 \mathrm{~h}$ at $\mathrm{rt}$, after which it was poured into aq. sat. $\mathrm{NaHCO}_{3} / \mathrm{Na}_{2} \mathrm{~S}_{2} \mathrm{O}_{3}(1: 1,30 \mathrm{~mL})$ and diluted with $\mathrm{CH}_{2} \mathrm{Cl}_{2}$. The biphasic mixture was stirred for $1 \mathrm{~h}$ and subsequently extracted with $\mathrm{CH}_{2} \mathrm{Cl}_{2}(3 \times 20 \mathrm{~mL})$. The combined organic layers were dried over $\mathrm{MgSO}_{4}$, filtered and all volatiles were removed in vacuo. The crude diketone was dissolved in $\mathrm{CH}_{2} \mathrm{Cl}_{2}(1.0 \mathrm{~mL})$ and the solution was cooled to $-78{ }^{\circ} \mathrm{C} . \mathrm{BCl}_{3}\left(1 \mathrm{M}\right.$ in $\mathrm{CH}_{2} \mathrm{Cl}_{2}, 75.0$ $\mu \mathrm{L}, 75.0 \mu \mathrm{mol}, 3.00$ equiv.) was added and the deep red mixture was slowly warmed to -20 ${ }^{\circ} \mathrm{C}$. The reaction was quenched by addition of phosphate-puffer $(p \mathrm{H}=7)$ and the aqueous layers was extracted with $\mathrm{CH}_{2} \mathrm{Cl}_{2}(3 \times 10 \mathrm{~mL})$. The combined organic layers were dried over $\mathrm{MgSO} 4$, filtered and all volatiles were removed in vacuo. The crude product was purified by flash chromatography on silica (3:1 $n$-pentane/EtOAc) to yield diketon 4 (6 mg, $19.6 \mu \mathrm{mol}, 75 \%)$ as yellowish powder.

1H-NMR: (500 MHz, acetone- $\left.d_{6}\right) \delta=7.51(\mathrm{t}, J=8.3 \mathrm{~Hz}, 1 \mathrm{H}, \mathrm{H}-3), 6.61(\mathrm{dd}, J=8.3,1.1 \mathrm{~Hz}$, $1 \mathrm{H}, \mathrm{H}-2), 6.56$ (dd, $J=8.3,0.8 \mathrm{~Hz}, 1 \mathrm{H}, \mathrm{H}-4), 4.83$ (dd, $J=12.9,10.4 \mathrm{~Hz}, 1 \mathrm{H}, \mathrm{H}-4 \mathrm{a}), 3.80$ (dd, $J=12.6,0.9 \mathrm{~Hz}, 1 \mathrm{H}, \mathrm{H}-5 \mathrm{a}), 3.74$ (s, 3H, H-11), 3.23 (dq, $J=10.4,6.8 \mathrm{~Hz}, 1 \mathrm{H}, \mathrm{H}-5), 1.33$ (d, $J$ $=6.9 \mathrm{~Hz}, 3 \mathrm{H}, \mathrm{H}-9)$ ppm. ${ }^{13} \mathrm{C}-\mathrm{NMR}:\left(126 \mathrm{MHz}\right.$, acetone- $\left.d_{6}\right) \delta=208.5$ (C-6), 197.3 (C-8), 169.9 (C-10), 163.2 (C-1), 162.4 (C-4a), 139.1 (C-3), 110.8 (C-2), 108.8 (C-8a), 108.8 (C-4), 82.7 (C-5a), 80.1 (C-7), 58.0 (C-7a), 53.7 (C-11), 48.6 (C-5), 12.3 (C-9) ppm. HRMS (ESI+): m/z calc. for $\mathrm{C}_{15} \mathrm{H}_{16} \mathrm{O}_{7} \mathrm{Na}[\mathrm{M}+\mathrm{Na}]^{+}$: 331.0788, found: 331.0796 . 


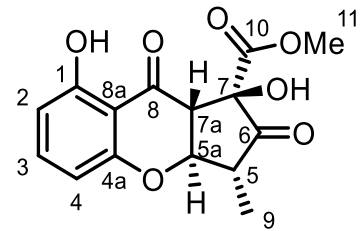

4

Reported Preussochromone F

\begin{tabular}{|c|c|c|c|c|}
\hline No & $\begin{array}{c}\text { Isolated product }^{[3]} \\
\text { aceton-d6 } \\
(600 \mathrm{MHz}) \\
\delta \mathrm{H}(\mathbf{J} \text { in } \mathrm{Hz})\end{array}$ & $\begin{array}{c}\text { synthetic product } 4 \\
\text { aceton-d } \\
(500 \mathrm{MHz}) \\
\delta \mathrm{H}(\mathrm{J} \text { in } \mathbf{H z})\end{array}$ & $\begin{array}{c}\text { Isolated product }{ }^{[3]} \\
\text { aceton-d }(150 \mathrm{MHz}) \\
\delta \mathrm{C}\end{array}$ & $\begin{array}{c}\text { synthetic product } \\
4 \\
\text { aceton- } d_{6}(125 \mathrm{MHz}) \\
\delta \mathrm{C}\end{array}$ \\
\hline 1 & & & 162.8 & 163.2 \\
\hline 2 & $6.49, \mathrm{~d}(8.0)$ & $6.61, \mathrm{dt}(8.3,1.1)$ & 110.4 & 110.8 \\
\hline 3 & $7.43, \mathrm{t}(8.0)$ & $7.51, \mathrm{t}(8.3)$ & 139.3 & 139.1 \\
\hline 4 & $6.46, \mathrm{~d}(8.0)$ & $6.56, \mathrm{dt}(8.3,0.8)$ & 108.4 & 108.8 \\
\hline $4 \mathbf{a}$ & & & 162.0 & 162.4 \\
\hline 5 & $3.13, \mathrm{~m}$ & $3.23, \mathrm{dq}(10.4,6.8)$ & 49.9 & 48.6 \\
\hline $5 \mathbf{a}$ & 5.31, dd (4.0) & 4.83, dd $(12.9,10.4)$ & 79.5 & 82.7 \\
\hline 6 & & & 210.5 & 208.5 \\
\hline 7 & & & 79.9 & 80.1 \\
\hline $7 \mathbf{a}$ & $3.83, \mathrm{~d}(4.0)$ & $3.80, \mathrm{dd}(12.6,0.9)$ & 53.7 & 58.0 \\
\hline 8 & & & 196.2 & 197.3 \\
\hline $8 \mathbf{a}$ & & & 109.4 & 108.8 \\
\hline 9 & $1.33, \mathrm{~d}(7.0)$ & $1.33, \mathrm{~d}(6.9)$ & 7.8 & 12.3 \\
\hline 10 & & & 171.5 & 169.9 \\
\hline 11 & $3.82, \mathrm{~s}$ & $3.74, \mathrm{~s}$ & 53.8 & 53.7 \\
\hline $\begin{array}{l}\text { OH-1 } \\
\text { OH-6 }\end{array}$ & $11.69, \mathrm{~s}$ & --- & & \\
\hline OH-7 & $5.69, \mathrm{~s}$ & --- & & \\
\hline
\end{tabular}




\subsection{Methyl (R)-2-diazo-4-((S)-5-methoxy-4-oxochroman-2-yl)-3-oxopentanoate}

(20)

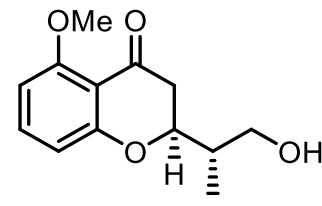

11
1) IBX, EtOAc, reflux

$5 \mathrm{~h}$

2) $\mathrm{MDA}, \mathrm{SnCl}_{2}, \mathrm{CH}_{2} \mathrm{Cl}_{2}$

$r t, 16 \mathrm{~h}$

3) ADMP, NMM, THF $0^{\circ} \mathrm{C} \rightarrow \mathrm{rt}, 30 \mathrm{~min}$

$77 \%$ over three steps

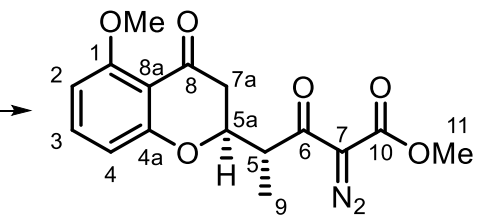

20

Alcohol 11 (2.07 g, 8.76 mmol, 1.00 equiv) was dissolved in EtOAc (219 mL) and IBX (4.91 g, $17.5 \mathrm{mmol}, 2.00$ equiv) was added. The suspension was heated to reflux and stirred for $5 \mathrm{~h}$. The resulting suspension was cooled to $0{ }^{\circ} \mathrm{C}$ and $\mathrm{Et}_{2} \mathrm{O}(219 \mathrm{~mL})$ was added. After stirring for 30 min, the suspension was filtered through a pad of $\mathrm{Na}_{2} \mathrm{SO}_{4}$ and the solvent was removed in vacuo to give the aldehyde as an orange oil. In a separate flask, $\mathrm{SnCl}_{2}$ (829 $\mathrm{mg}, 4.38 \mathrm{mmol}, 0.50$ equiv) was suspended in $\mathrm{CH}_{2} \mathrm{Cl}_{2}$ (44 mL) and methyl diazoacetate (MDA, $11.5 \mathrm{M}$ in $\mathrm{CH}_{2} \mathrm{Cl}_{2}, 1.52 \mathrm{~mL}$, $17.5 \mathrm{mmol}, 2.00$ equiv) and a solution of the freshly prepared aldehyde in $\mathrm{CH}_{2} \mathrm{Cl}_{2}(22 \mathrm{~mL})$ were added successively, which led to a vigorous gas formation. The suspension was stirred at $\mathrm{rt}$ overnight and quenched by addition of aq. sat. $\mathrm{NH}_{4} \mathrm{~F}(200 \mathrm{~mL})$. The layers were separated and the aqueous layer was extracted with $\mathrm{CH}_{2} \mathrm{Cl}_{2}(3 \times 100 \mathrm{~mL})$. The combined organic layers were dried over $\mathrm{MgSO}_{4}$, filtered and all volatiles were removed in vacuo. Residual water was coevaporated using toluene $(3 \times 20 \mathrm{~mL})$ and the orange residue was dissolved in THF ( $88 \mathrm{~mL})$. 2-Azido-1,3-dimethylimidazolinium hexafluorophosphate (ADMP, $2.75 \mathrm{~g}, 9.64 \mathrm{mmol}$, 1.10 equiv) was added and the mixture was cooled to $0{ }^{\circ} \mathrm{C}$. $\mathrm{N}$-methyl morpholine (NMM, $1.02 \mathrm{~mL}, 9.20 \mathrm{mmol}, 1.05$ equiv) was added and the mixture was stirred for $10 \mathrm{~min}$ at $\mathrm{rt}$. The reaction was quenched by addition of $\mathrm{H}_{2} \mathrm{O}(200 \mathrm{~mL})$ and the aqueous layers was extracted with EtOAc (3x $100 \mathrm{~mL}$ ). The combined organic layers were washed with brine, dried over $\mathrm{MgSO}_{4}$, filtered and all volatiles were removed in vacuo. The crude product was purified by flash chromatography on silica (1:1 n-pentane/EtOAc) to yield diazoester 20 (2.24 g, $6.74 \mathrm{mmol}$, $77 \%$ ) as slightly yellow foam.

Note: Diazoester 20 is badly soluble in chlorinated solvents and toluene but is well soluble in polar solvents like THF, EtOAc and $\mathrm{MeOH}$.

TLC: $\mathrm{R}_{f}=0.23\left(1: 1 n\right.$-pentane/EtOAc). ${ }^{1} \mathbf{H}$-NMR: $\left(500 \mathrm{MHz}, \mathrm{CDCl}_{3}\right) \delta=7.35(\mathrm{t}, J=8.3 \mathrm{~Hz}$, 1H, H-3), 6.57 (d, $J=8.4 \mathrm{~Hz}, 1 \mathrm{H}, \mathrm{H}-4), 6.50$ (d, $J=8.2 \mathrm{~Hz}, 1 \mathrm{H}, \mathrm{H}-2$ ), 4.63 (ddd, $J=12.1,7.0$, $3.3 \mathrm{~Hz}, 1 \mathrm{H}, \mathrm{H}-5 \mathrm{a}), 4.00$ (p, J=6.9 Hz, 1H, H-5), 3.90 (s, 3H, -OCH3), 3.83 (s, 3H, H-11), 2.78 (dd, $J=16.4,12.1 \mathrm{~Hz}, 1 \mathrm{H}, \mathrm{H}-7 \mathrm{a}), 2.70$ (dd, $J=16.4,3.3 \mathrm{~Hz}, 1 \mathrm{H}, \mathrm{H}-7 \mathrm{a}$ '), 1.32 (d, $J=6.9 \mathrm{~Hz}$, 
3H, H-9) ppm. ${ }^{13}$ C-NMR: (126 MHz, $\left.\mathrm{CDCl}_{3}\right) \delta=192.9$ (C-6), 190.6 (C-8), 163.1 (C-4a), 161.4 (C-10), 160.9 (C-1), 136.0 (C-3), 111.6 (C-8a), 110.2 (C-4), 104.2 (C-2), 77.9 (C-5a), 56.3 ($\mathrm{OCH}_{3}$ ), 52.5 (C-11), 45.6 (C-5), 42.4 (C-7a), 12.8 (C-9) ppm. HRMS (ESI+): m/z calc. for $\mathrm{C}_{16} \mathrm{H}_{16} \mathrm{~N}_{2} \mathrm{O}_{6} \mathrm{Na}[\mathrm{M}+\mathrm{Na}]^{+}:$355.0901, found: 355.0912. FT-IR: (neat): $\tilde{v}=2146(\mathrm{w}), 1720(\mathrm{w})$, 1679 (m), 1604 (m), 1575 (w), 1473 (m), 1441 (w), 1380 (w), 1333 (w), 1311 (w), 1280 (w), 1257 (m), 1234 (w), 1134 (w), 1101 (w), 1082 (m), 1049 (w), 999 (w), 827 (s), 739 (w), 556 (s) $\mathrm{cm}^{-1} \cdot[\alpha]_{\mathrm{D}}^{20}:-13.0\left(\mathrm{c} 0.5, \mathrm{CHCl}_{3}\right)$.

\subsection{Methyl (R)-4-((S)-5-acetoxy-4-oxochroman-2-yl)-2-diazo-3-oxopentanoate} (21)

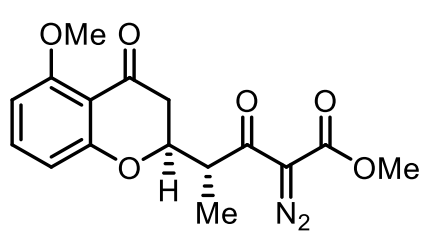

20

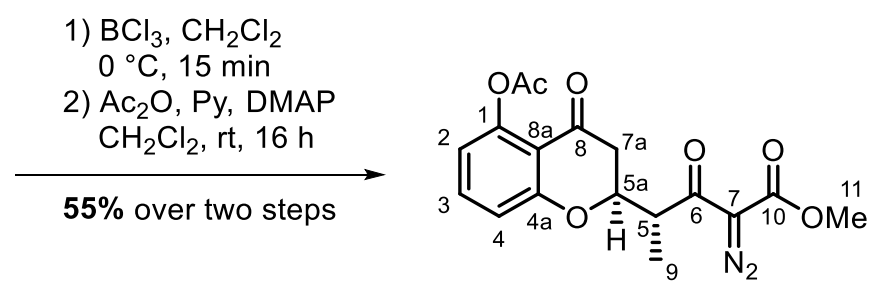

21

Methyl ether 20 (500 mg, 1.50 mmol, 1.00 equiv) was suspended in $\mathrm{CH}_{2} \mathrm{Cl}_{2}$ (45 mL) and cooled to $0{ }^{\circ} \mathrm{C} . \mathrm{BCl}_{3}\left(1 \mathrm{M}\right.$ in $\mathrm{CH}_{2} \mathrm{Cl}_{2}, 2.26 \mathrm{~mL}, 2.26 \mathrm{mmol}, 1.50$ equiv) was added dropwise and the deep red mixture was stirred for $15 \mathrm{~min}$ at $0{ }^{\circ} \mathrm{C}$. The reaction was quenched by addition of ice cold $\mathrm{H}_{2} \mathrm{O}(50 \mathrm{~mL})$ and the layers were separated. The aqueous layer was extracted with $\mathrm{CH}_{2} \mathrm{Cl}_{2}$ ( $3 \times 50 \mathrm{~mL}$ ) and the combined organic layers were dried over $\mathrm{MgSO}_{4}$, filtered and all volatiles were removed in vacuo. The resulting deep red oil was dissolved in $\mathrm{CH}_{2} \mathrm{Cl}_{2}(15 \mathrm{~mL})$ and $\mathrm{Ac}_{2} \mathrm{O}$ (1.13 mL, $12.0 \mathrm{mmol}, 8.00$ equiv), pyridine (1.21 mL, $15.0 \mathrm{mmol}, 10.0$ equiv) and DMAP (9.2 $\mathrm{mg}, 75.0 \mu \mathrm{mol}, 5 \mathrm{~mol} \%)$ were added successively. The resulting solution was stirred overnight at $\mathrm{rt}$ and then quenched by addition of $\mathrm{H}_{2} \mathrm{O}(100 \mathrm{~mL})$. The layers were separated and the aqueous layer was extracted with $\mathrm{CH}_{2} \mathrm{Cl}_{2}(3 \times 50 \mathrm{~mL})$ and the combined organic layers were dried over $\mathrm{MgSO}_{4}$, filtered and all volatiles were removed in vacuo. The crude product was purified by flash chromatography on silica (3:1 n-pentane/EtOAc) to yield acetate 21 (299 mg, $0.83 \mathrm{mmol}, 55 \%)$ as pale yellow, clear oil.

TLC: $\mathbf{R}_{f}=0.17$ (6:1 $n$-pentane/EtOAc). ${ }^{1} \mathbf{H}$-NMR: $\left(500 \mathrm{MHz}, \mathrm{CDCl}_{3}\right) \delta=7.42(\mathrm{dd}, J=8.5$, $7.9 \mathrm{~Hz}, 1 \mathrm{H}, \mathrm{H}-3), 6.89$ (dd, $J=8.5,1.1 \mathrm{~Hz}, 1 \mathrm{H}, \mathrm{H}-4), 6.65$ (dd, $J=7.9,1.1 \mathrm{~Hz}, 1 \mathrm{H}, \mathrm{H}-2), 4.70$ (ddd, $J=12.8,6.4,2.9 \mathrm{~Hz}, 1 \mathrm{H}, \mathrm{H}-5 \mathrm{a}), 3.98$ (p, $J=6.8 \mathrm{~Hz}, 1 \mathrm{H}, \mathrm{H}-5$ ), 3.84 (s, 3H, H-11), 2.79 (dd, $J=16.5,12.8 \mathrm{~Hz}, 1 \mathrm{H}, \mathrm{H}-7 \mathrm{a}), 2.65$ (dd, $J=16.5,2.9 \mathrm{~Hz}, 1 \mathrm{H}, \mathrm{H}-7 \mathrm{a}$ '), 2.36 (s, 3H, $\left.H_{3} \mathrm{CC}(\mathrm{O}) \mathrm{O}-\right), 1.32$ (d, $\left.J=6.9 \mathrm{~Hz}, 3 \mathrm{H}, \mathrm{H}-9\right)$ ppm. ${ }^{13} \mathrm{C}-\mathbf{N M R}:\left(126 \mathrm{MHz}, \mathrm{CDCl}_{3}\right) \delta=192.6$ (C-6), 190.1 (C-8), 169.7 (H3CC(O)O-), 162.5 (C-4a), 161.5 (C-10), 150.3 (C-1), 135.7 (C-3), 
116.3 (C-4), 116.2 (C-2), 114.0 (C-8a), 78.0 (C-5a), 52.5 (C-11), 45.6 (C-5), 41.7 (C-7a), 21.2 ( $\left.\mathrm{H}_{3} \mathrm{CC}(\mathrm{O}) \mathrm{O}-\right), 12.4$ (C-9) ppm. HRMS (ESI+): $\mathrm{m} / \mathrm{z}$ calc. for $\mathrm{C}_{17} \mathrm{H}_{16} \mathrm{~N}_{2} \mathrm{O}_{7} \mathrm{Na}[\mathrm{M}+\mathrm{Na}]^{+}$: 383.0850, found: 383.0859. FT-IR: (neat): $\tilde{v}=2956(\mathrm{w}), 2878(\mathrm{w}), 2145(\mathrm{~m}), 1767(\mathrm{~m}), 1716$ (w), 1690 (s), 1648 (w), 1612 (m), 1571 (w), 1465 (s), 1438 (w), 1366 (w), 1304 (m), 1280 (w), 1188 (s), 1128 (w), 1049 (m), 995 (w), 905 (m), 874 (m), 815 (m), 785 (w), 740 (m), 706 (w), $638(\mathrm{w}), 575(\mathrm{~m}), 550(\mathrm{w}), 528(\mathrm{w}) \mathrm{cm}^{-1}[\boldsymbol{\alpha}]_{\mathrm{D}}^{20}:+28.7\left(\mathrm{c} 0.5, \mathrm{CHCl}_{3}\right)$.

\subsection{Methyl (R)-4-((S)-5-hydroxy-4-oxochroman-2-yl)-2,3-dioxopentanoate (22)}

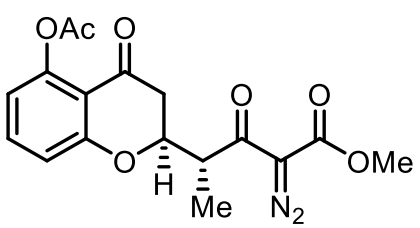

21

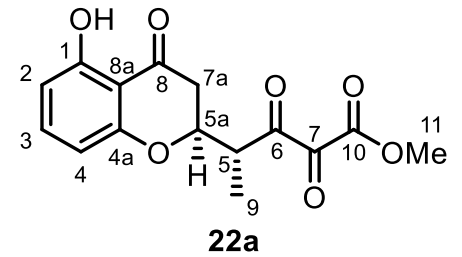

$\mathbf{7 8 \%}$ over two steps

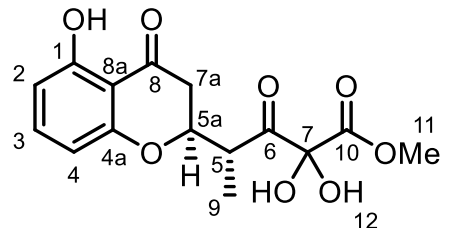

22b

Acetate 21 (493 mg, $1.37 \mathrm{mmol}, 1.00$ equiv) was dissolved in a freshly prepared DMDO solution in acetone ( $\sim 0.06 \mathrm{M}, 114 \mathrm{~mL}, 6.84 \mathrm{mmol}, 5.00$ equiv) and the resulting solution was stirred at rt overnight. All volatiles were removed in vacuo and the residual water was removed by coevaporation with toluene $(3 \mathrm{x} 50 \mathrm{~mL})$. The residue was dissolved in $\mathrm{MeOH}(50 \mathrm{~mL})$ and $\mathrm{AcCl}(97.6 \mu \mathrm{L}, 1.37 \mathrm{mmol}, 1.00$ equiv) was added and the reaction was stirred at $\mathrm{rt}$ overnight. Another portion of $\mathrm{AcCl}$ was added $(97.6 \mu \mathrm{L}, 1.37 \mathrm{mmol}, 1.00$ equiv) and the mixture was stirred overnight. $\mathrm{H}_{2} \mathrm{O}(200 \mathrm{~mL})$ was added and the mixture was extracted with EtOAc $(3 \mathrm{x} 100$ $\mathrm{mL}$ ). The combined organic layers were washed with brine, dried over $\mathrm{MgSO}_{4}$, filtered and all volatiles were removed in vacuo. The crude product was purified by flash chromatography on silica (2:1 n-pentane/EtOAc) to yield tricarbonyl $22(327 \mathrm{mg}, 1.07 \mathrm{mmol}, 78 \%, 1: 1$ hydrate/victricarbonyl) as deep yellow, sticky oil.

Note: The Tricarbonyl was always isolated as a mixture of the vic-tricarbonyl 22a and its hydrate form $22 \boldsymbol{b}$. The ratio varied from experiment to experiment, but it had no effect on the subsequent cyclization step. For the sake of clarity, NMR-data are given separately, although they are not separable by chromatography.

TLC: $\mathbf{R}_{f}=0.40$ (1:1 n-pentane/EtOAc). FT-IR: (neat): $\tilde{v}=3435(\mathrm{w}), 2956(\mathrm{w}), 1730(\mathrm{~m}), 1626$ (s), 1577 (m), 1460 (s), 1351 (m), 1296 (w), 1254 (w), 1218 (s), 1157 (w), 1101 (w), 1054 (s), 
$966(\mathrm{~m}), 901(\mathrm{w}), 872(\mathrm{w}), 796(\mathrm{~m}), 726(\mathrm{~s}), 651(\mathrm{w}), 631(\mathrm{w}), 579(\mathrm{w}), 521(\mathrm{w}), 476(\mathrm{w}), 455$ (w), $430(\mathrm{w}) \mathrm{cm}^{-1} \cdot[\boldsymbol{\alpha}]_{\mathrm{D}}^{20}:+49.1\left(\mathrm{c} 0.5, \mathrm{CHCl}_{3}\right)$.

Tricarbonyl 22a: HRMS (ESI+): $\mathrm{m} / \mathrm{z}$ calc. for $\mathrm{C}_{15} \mathrm{H}_{14} \mathrm{O}_{7} \mathrm{Na}[\mathrm{M}+\mathrm{Na}]^{+}:$329.0643, found: 329.0643. ${ }^{1}$ H-NMR: $\left(500 \mathrm{MHz}, \mathrm{CDCl}_{3}\right) \delta=7.35$ (t, $\left.J=8.3 \mathrm{~Hz}, 1 \mathrm{H}, \mathrm{H}-3\right), 6.52(\mathrm{dd}, J=6.9$, $1.0 \mathrm{~Hz}, 1 \mathrm{H}, \mathrm{H}-2), 6.41$ (dd, $J=8.2,0.9 \mathrm{~Hz}, 1 \mathrm{H}, \mathrm{H}-4), 4.65$ (ddd, $J=11.9,7.2,3.4 \mathrm{~Hz}, 1 \mathrm{H}, \mathrm{H}-$ 5a), 3.85 (s, 3H, H-11), 3.48 (qd, $J=7.0,3.1 \mathrm{~Hz}, 1 \mathrm{H}, \mathrm{H}-5), 2.80$ (dd, $J=17.2,11.9 \mathrm{~Hz}, 1 \mathrm{H}, \mathrm{H}-$ 7a), 2.71 (dd, $J=17.2,3.4 \mathrm{~Hz}, 1 \mathrm{H}, \mathrm{H}-7 \mathrm{a}$ ) $), 1.36$ (d, $J=7.0 \mathrm{~Hz}, 3 \mathrm{H}, \mathrm{H}-9)$ ppm. ${ }^{13}$ C-NMR: (126 $\mathrm{MHz}, \mathrm{CDCl}_{3}$ ) $\delta=204.5$ (C-6), 197.0 (C-8), 181.9 (C-7), 169.2 (C-10), 162.3 (C-1), 160.7 (C4a), 138.4 (C-3), 110.1 (C-2), 108.2 (C-8a), 107.4 (C-4), 77.9 (C-5a), 54.2 (C-11), 44.3 (C-5), 39.8 (C-7a), 14.2 (C-9) ppm.

Hydrate 22b: HRMS (ESI+): m/z calc. for $\mathrm{C}_{15} \mathrm{H}_{16} \mathrm{O}_{8} \mathrm{Na}$ [M+Na] $]^{+}: 347.0737$, found: 347.0750. ${ }^{1}$ H-NMR: $\left(500 \mathrm{MHz}, \mathrm{CDCl}_{3}\right) \delta=7.35(\mathrm{t}, J=8.3 \mathrm{~Hz}, 1 \mathrm{H}, \mathrm{H}-3), 6.54(\mathrm{dd}, J=6.9,0.9 \mathrm{~Hz}, 1 \mathrm{H}$, H-2), 6.38 (dd, $J=8.2,0.9 \mathrm{~Hz}, 1 \mathrm{H}, \mathrm{H}-4), 4.79$ (ddd, $J=13.5,5.8,2.9 \mathrm{~Hz}, 1 \mathrm{H}, \mathrm{H}-5 \mathrm{a}), 3.88$ (s, $3 \mathrm{H}, \mathrm{H}-11$ ), 3.78 (qd, $J=7.1,5.8 \mathrm{~Hz}, 1 \mathrm{H}, \mathrm{H}-5), 2.90$ (dd, $J=17.0,13.5 \mathrm{~Hz}, 1 \mathrm{H}, \mathrm{H}-7 \mathrm{a}), 2.69$ (dd, $J=17.0,2.9 \mathrm{~Hz}, 1 \mathrm{H}, \mathrm{H}-7 \mathrm{a}$ ), 1.33 (d, $J=7.1 \mathrm{~Hz}, 3 \mathrm{H}, \mathrm{H}-9)$ ppm. ${ }^{13} \mathbf{C}-\mathbf{N M R}:\left(126 \mathrm{MHz}, \mathrm{CDCl}_{3}\right)$ $\delta=199.3$ (C-6), 197.6 (C-8), 162.3 (C-1), 160.9 (C-10), 160.4 (C-4a), 138.5 (C-3), 110.5 (C2), 108.1 (C-8a), 107.2 (C-4), 93.4 (C-7), 77.0 (C-5a), 53.6 (C-11), 44.8 (C-5), 38.8 (C-7a), 10.1 (C-9) ppm.

\subsection{6 (-)-Preussochromone F (23)}
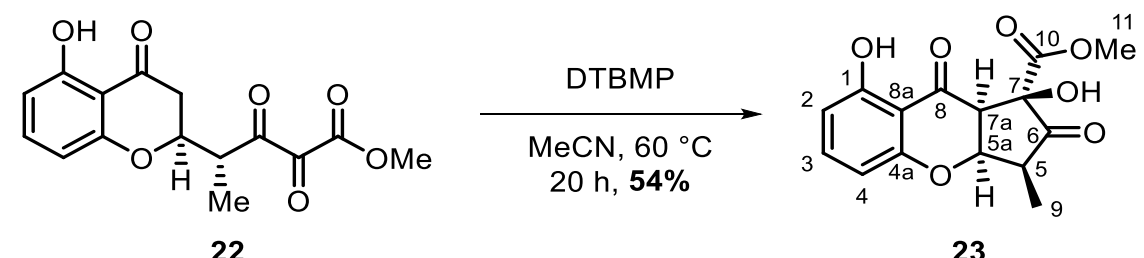

Tricarbonyl 23 (92.0 mg, $304 \mu \mathrm{mol}, 1.00$ eq.) and 2,6-di-t-butyl-4-methyl pyridine (DTBMP, $67.8 \mathrm{mg}, 330 \mu \mathrm{mol}, 1.10$ equiv) were dissolved in $\mathrm{MeCN}(6.0 \mathrm{~mL})$ and the solution was warmed to $60{ }^{\circ} \mathrm{C}$ for $20 \mathrm{~h}$. The mixture was cooled to $\mathrm{rt}$ and silica was added. All volatiles were removed and the crude product which was adsorbed on silica was purified by flash chromatography on silica (3:1 n-pentane/EtOAc) to yield Preussochromone F 23 (50.0 mg, $163 \mu \mathrm{mol}, 54 \%)$ as yellow solid.

TLC: $\mathbf{R}_{f}=0.50\left(1: 1 n\right.$-pentane/EtOAc). ${ }^{1} \mathbf{H}$-NMR: $\left(500 \mathrm{MHz}\right.$, acetone- $\left.d_{6}\right) \delta=11.71(\mathrm{~s}, 1 \mathrm{H}, 1$ $\mathrm{OH}), 7.44(\mathrm{t}, J=8.3 \mathrm{~Hz}, 1 \mathrm{H}, \mathrm{H}-3), 6.50$ (dd, $J=8.4,0.9 \mathrm{~Hz}, 1 \mathrm{H}, \mathrm{H}-2), 6.46$ (dd, $J=8.2,1.0 \mathrm{~Hz}$, $1 \mathrm{H}, \mathrm{H}-3), 5.74$ (d, $J=1.1 \mathrm{~Hz}, 1 \mathrm{H}, 7-\mathrm{OH}), 5.32$ (t, $J=4.2 \mathrm{~Hz}, 1 \mathrm{H}, \mathrm{H}-5 \mathrm{a}), 3.84$ (d, $J=4.3 \mathrm{~Hz}$, $1 \mathrm{H}, \mathrm{H}-7 \mathrm{a}), 3.83$ (s, 3H, H-11), 3.14 (qd, $J=6.9,4.2 \mathrm{~Hz}, 1 \mathrm{H}, \mathrm{H}-5), 1.35$ (d, $J=6.9 \mathrm{~Hz}, 3 \mathrm{H}, \mathrm{H}-$ 
9) ppm. ${ }^{13}$ C-NMR: (126 MHz, acetone- $\left.d_{6}\right) \delta=210.6$ (C-6), 196.2 (C-8), 171.5 (C-10), 162.8 (C-1), 161.9 (C-4a), 139.3 (C-3), 110.4 (C-2), 110.3 (C-8a), 108.5 (C-4), 79.9 (C-7), 79.5 (C5a), 53.8 (C-11), 53.7 (C-7a), 49.9 (C-5), 7.8 (C-9) ppm. HRMS (ESI+): m/z calc. for $\mathrm{C}_{15} \mathrm{H}_{15} \mathrm{O}_{7}$ $[\mathrm{M}+\mathrm{H}]^{+}:$307.0812, found: 307.0827. FT-IR: $\tilde{v}=3402(\mathrm{w}), 2956(\mathrm{w}), 2923(\mathrm{w}), 2852(\mathrm{w}), 1742$ (s), 1626 (s), 1577 (w), 1462 (m), 1357 (m), 1271 (m), 1218 (s), 1136 (m), 1049 (s), 1024 (w), 998 (w), $967(\mathrm{~m}), 923(\mathrm{w}), 897(\mathrm{w}), 790(\mathrm{w}), 763(\mathrm{w}), 729(\mathrm{~s}), 689(\mathrm{w}), 665(\mathrm{~m}), 636(\mathrm{w}), 606$ (w), 589 (w), $496(w), 466(w) \mathrm{cm}^{-1}$. m.p.: $176^{\circ} \mathrm{C}\left(\mathrm{Et}_{2} \mathrm{O}\right) .[\boldsymbol{\alpha}]_{\mathrm{D}}^{\mathbf{2 0}}:-108$ (c 0.1, MeOH).

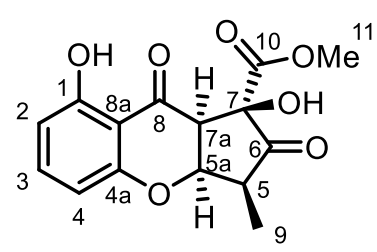

23

Preussochromone $\mathrm{F}$

\begin{tabular}{|c|c|c|c|c|}
\hline No & $\begin{array}{c}\text { Isolated product }^{[3]} \\
\text { aceton- } d_{6} \\
(600 \mathrm{MHz}) \\
\delta \mathrm{H}(\mathrm{J} \text { in } \mathrm{Hz}) \\
\end{array}$ & $\begin{array}{c}\text { synthetic product } 23 \\
\text { aceton- } d_{6} \\
(500 \mathrm{MHz}) \\
\delta \mathrm{H}(\mathrm{J} \text { in } \mathbf{H z}) \\
\end{array}$ & $\begin{array}{c}\text { Isolated product }{ }^{[3]} \\
\text { aceton- } d_{\sigma}(150 \mathrm{MHz}) \\
\delta \mathrm{C}\end{array}$ & $\begin{array}{c}\text { synthetic product } \\
23 \\
\text { aceton- } d_{6}(126 \mathrm{MHz}) \\
\delta \mathrm{C} \\
\end{array}$ \\
\hline 1 & & & 162.8 & 162.8 \\
\hline 2 & $6.49, \mathrm{~d}(8.0)$ & $6.50, \mathrm{dd}(8.4,0.9)$ & 110.4 & 110.4 \\
\hline 3 & $7.43, \mathrm{t}(8.0)$ & $7.44, \mathrm{t}(8.3)$ & 139.3 & 139.3 \\
\hline 4 & $6.46, \mathrm{~d}(8.0)$ & $6.46, \mathrm{dd}(8.0,0.9)$ & 108.4 & 108.5 \\
\hline $4 \mathbf{a}$ & & & 162.0 & 161.9 \\
\hline 5 & $3.13, \mathrm{~m}$ & $3.14, \mathrm{qd}(6.9,4.2)$ & 49.9 & 49.9 \\
\hline $5 \mathbf{a}$ & $5.31, \mathrm{dd}(4.0)$ & $5.32, \mathrm{t}(4.2)$ & 79.5 & 79.5 \\
\hline 6 & & & 210.5 & 210.6 \\
\hline 7 & & & 79.9 & 79.9 \\
\hline $7 \mathbf{a}$ & $3.83, \mathrm{~d}(4.0)$ & $3.84, \mathrm{~d}(4.3)$ & 53.7 & 53.8 \\
\hline 8 & & & 196.2 & 196.2 \\
\hline $8 \mathbf{a}$ & & & $109.4^{a}$ & 110.3 \\
\hline 9 & $1.33, \mathrm{~d}(7.0)$ & $1.35, \mathrm{~d}(7.0)$ & 7.8 & 7.8 \\
\hline 10 & & & 171.5 & 171.5 \\
\hline 11 & $3.82, \mathrm{~s}$ & $3.83, \mathrm{~s}$ & 53.8 & 53.7 \\
\hline OH-1 & $11.69, \mathrm{~s}$ & 11.71, s & & \\
\hline OH-6 & & & & \\
\hline OH-7 & $5.69, \mathrm{~s}$ & $5.74, \mathrm{~s}$ & & \\
\hline
\end{tabular}


2.17 (+)-Preussochromone E (24)

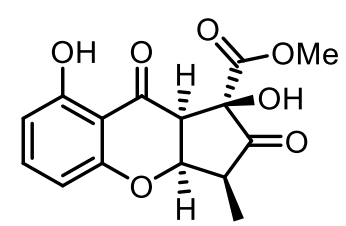

23

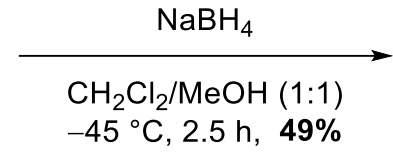

$-45^{\circ} \mathrm{C}, 2.5 \mathrm{~h}, 49 \%$

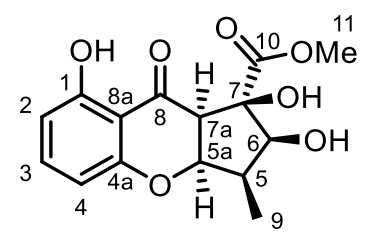

24

To a solution of preussochromone $\mathrm{F}(\mathbf{2 3})\left(10.0 \mathrm{mg}, 32.7 \mu \mathrm{mol}, 1.00\right.$ equiv.) in $\mathrm{CH}_{2} \mathrm{Cl}_{2} / \mathrm{MeOH}$ $(1: 1,1.0 \mathrm{~mL})$ was added $\mathrm{NaBH}_{4}(4.00 \mathrm{mg}, 106 \mu \mathrm{mol}, 3.20$ equiv. $)$ at $-45^{\circ} \mathrm{C}$. The reaction was stirred for $2.5 \mathrm{~h}$ before water was added and the mixture was warmed to room temperature. The aqueous layer was extracted with $\mathrm{CH}_{2} \mathrm{Cl}_{2}(2 \times 2 \mathrm{ml})$, dried over $\mathrm{Na}_{2} \mathrm{SO}_{4}$ and all volatiles were removed in vacuo. The crude product was purified by flash chromatography on silica ( $n$ pentane/ethyl acetate 1:1) to obtain preussochromone E (24) as colorless foam (5.00 mg, 16.1 $\mu \mathrm{mol}, 49 \%)$.

TLC: $\mathbf{R}_{f}=0.27\left(1: 1 n\right.$-pentane/EtOAc). ${ }^{1} \mathbf{H}$-NMR: $\left(500 \mathrm{MHz}\right.$, acetone- $\left.d_{6}\right) \delta=11.83(\mathrm{~s}, 1 \mathrm{H}, 1$ $\mathrm{OH}), 7.39$ (t, $J=8.3 \mathrm{~Hz}, 1 \mathrm{H}, \mathrm{H}-3), 6.42$ (dd, $J=8.3,0.8 \mathrm{~Hz}, 1 \mathrm{H}, \mathrm{H}-4), 6.41$ (dd, $J=8.3,0.9 \mathrm{~Hz}$, 1H, H-2), 4.91 (t, J = 4.4 Hz, 1H, H-5a), 4.54 (s, 1H, 7-OH), 4.36 (s, 1H, 6-OH), 4.31 (d, $J=$ $7.8 \mathrm{~Hz}, 1 \mathrm{H}, \mathrm{H}-6), 3.79$ (s, 3H, C-11), 3.46 (d, J=4.6 Hz, 1H, 7a), 2.54 (pd, J = 7.4, 4.2 Hz, 1H, H-5), 1.29 (d, $J=7.3 \mathrm{~Hz}, 3 \mathrm{H}, \mathrm{H}-9)$ ppm. ${ }^{13} \mathrm{C}-\mathbf{N M R}$ : (126 MHz, acetone- $\left.d_{6}\right) \delta=197.2(\mathrm{C}-8)$, 174.5 (C-10), 162.1 (C-1), 161.9 (C-4a), 138.2 (C-3), 109.6 (C-8a), 108.8 (C-2), 107.7 (C-4), 83.7 (C-5a), 83.0 (C-7), 77.7 (C-6), 56.0 (C-7a), 52.4 (C-11), 43.3 (C-5), 8.1 (C-9) ppm. HRMS (ESI+): $\mathrm{m} / \mathrm{z}$ calc. for $\mathrm{C}_{15} \mathrm{H}_{16} \mathrm{O}_{7} \mathrm{Na}[\mathrm{M}+\mathrm{Na}]^{+}: 331.0788$, found: 331.0802 . FT-IR: $\tilde{v}=3435(\mathrm{w})$, 2924 (w), 2852 (w), 1735 (m), 1626 (s), 1578 (w), 1461 (s), 1362 (m), 1219 (s), 1156 (w), 1107 (m), 1058 (m), $980(\mathrm{w}), 955(\mathrm{w}), 919(\mathrm{w}), 824(\mathrm{w}), 793(\mathrm{w}), 776(\mathrm{w}), 734(\mathrm{w}), 714(\mathrm{w}), 633$

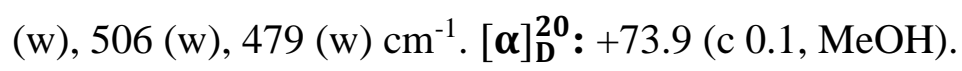




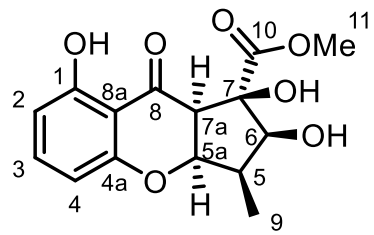

24

Preussochromone E

\begin{tabular}{|c|c|c|c|c|}
\hline No & $\begin{array}{c}\text { Isolated product }^{[3]} \\
{\text { aceton- } \boldsymbol{d}_{\boldsymbol{\sigma}}}_{(\mathbf{6 0 0} \mathrm{MHz})} \\
\delta \mathrm{H}(\mathrm{J} \text { in } \mathrm{Hz})\end{array}$ & $\begin{array}{c}\text { synthetic product } 24 \\
\text { aceton- } \boldsymbol{d}_{6} \\
(\mathbf{5 0 0} \mathrm{MHz}) \\
\delta \mathrm{H}(\mathbf{J} \text { in } \mathbf{H z})\end{array}$ & $\begin{array}{c}\text { Isolated product }{ }^{[3]} \\
\text { aceton- } d_{6}(150 \mathrm{MHz}) \\
\delta \mathrm{C}\end{array}$ & $\begin{array}{c}\text { synthetic product } \\
24 \\
\text { aceton- } d_{6}(126 \mathrm{MHz}) \\
\delta \mathrm{C}\end{array}$ \\
\hline $\mathbf{1}$ & & & 162.0 & $162.1(\mathrm{C}-1$ \\
\hline 2 & $6.41, \mathrm{~d}(8.0)$ & $6.41, \mathrm{dd}(8.3,0.8)$ & 108.8 & 108.8 \\
\hline 3 & $7.39, \mathrm{t}(8.0)$ & $7.39(\mathrm{t}, 8.3)$ & 138.3 & 138.2 \\
\hline 4 & $6.41, \mathrm{~d}(8.0)$ & $6.42,(8.3,0.8)$ & 107.8 & 107.7 \\
\hline $\mathbf{4 a}$ & & & 161.9 & 161.9 \\
\hline 5 & $2.53, \mathrm{~m}$ & $2.54, \operatorname{pd}(7.4,4.2)$ & 43.3 & 43.3 \\
\hline $5 \mathbf{a}$ & $4.91, \mathrm{dd}(4.0)$ & $4.91, \mathrm{t}(4.4)$ & 83.8 & 83.7 \\
\hline 6 & $4.29, \mathrm{~d}(7.5)$ & $4.31, \mathrm{~d}(7.8)$ & 77.7 & 77.7 \\
\hline 7 & & & 83.8 & 83.0 \\
\hline $7 \mathbf{a}$ & $3.45, \mathrm{~d}(4.0)$ & $3.46, \mathrm{~d}(4.6)$ & 56.1 & 56.0 \\
\hline 8 & & & 197.2 & 197.2 \\
\hline $8 \mathbf{a}$ & & & 109.7 & 109.6 \\
\hline 9 & $1.27, \mathrm{~d}(7.0)$ & $1.29, \mathrm{~d}(7.3)$ & 8.2 & 8.1 \\
\hline 10 & & & 174.5 & 174.5 \\
\hline 11 & $3.78, \mathrm{~s}$ & & 52.5 & 52.4 \\
\hline OH-1 & $11.83, \mathrm{~s}$ & $11.83, \mathrm{~s}$ & & \\
\hline OH-6 ${ }^{a}$ & $4.37, \mathrm{~s}^{b}$ & $4.36, \mathrm{~s}$ & & \\
\hline OH-7 ${ }^{a}$ & $4.53, \mathrm{~s}^{b}$ & $4.54, \mathrm{~s}$ & & \\
\hline
\end{tabular}

${ }^{a} \mathrm{OH}$ Signals exchange with HDO present in acetone- $d_{6}$, giving more than one set of signals. This also broadens the signal of H-6. ${ }^{b}$ authors did a wrong assignment of OH-6 and OH-7, which are broad singuletts in their spectra. 


\section{$3{ }^{1} \mathrm{H},{ }^{13} \mathrm{C}-\mathrm{NMR}$}

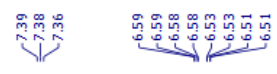

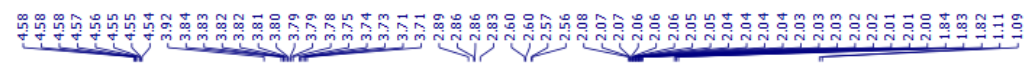

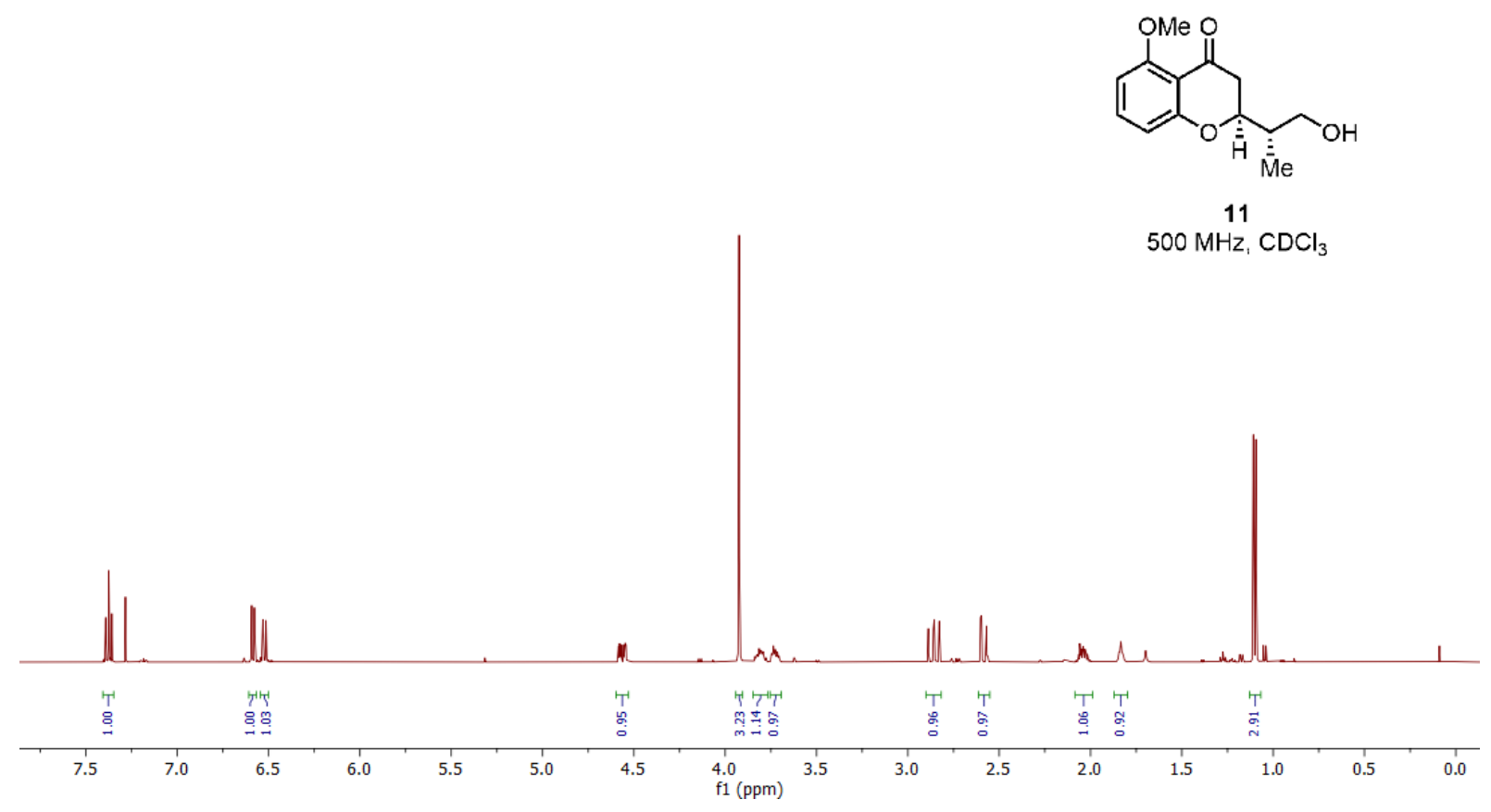

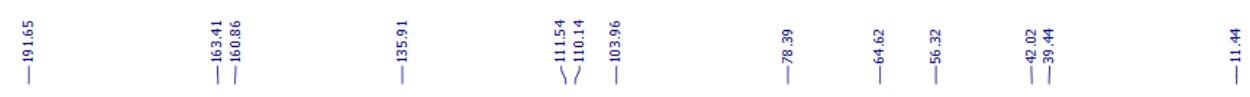

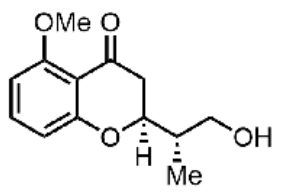

11

$126 \mathrm{MHz}_{1} \mathrm{CDCl}_{3}$

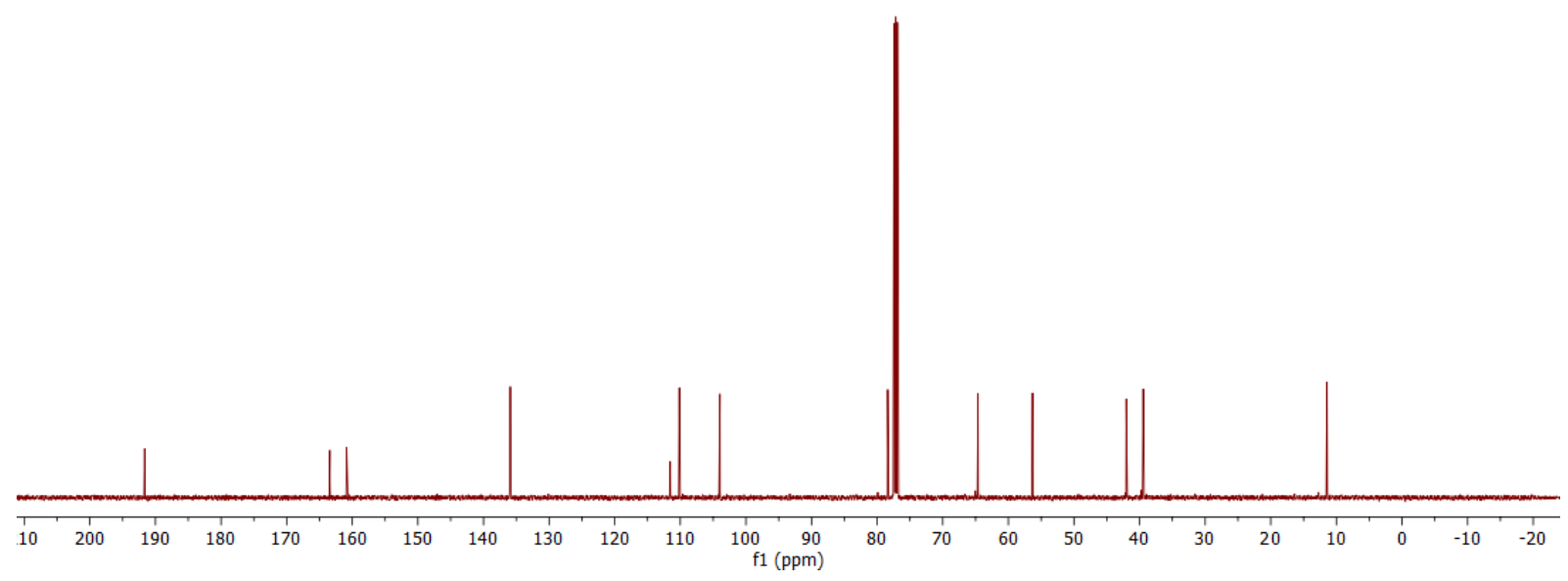



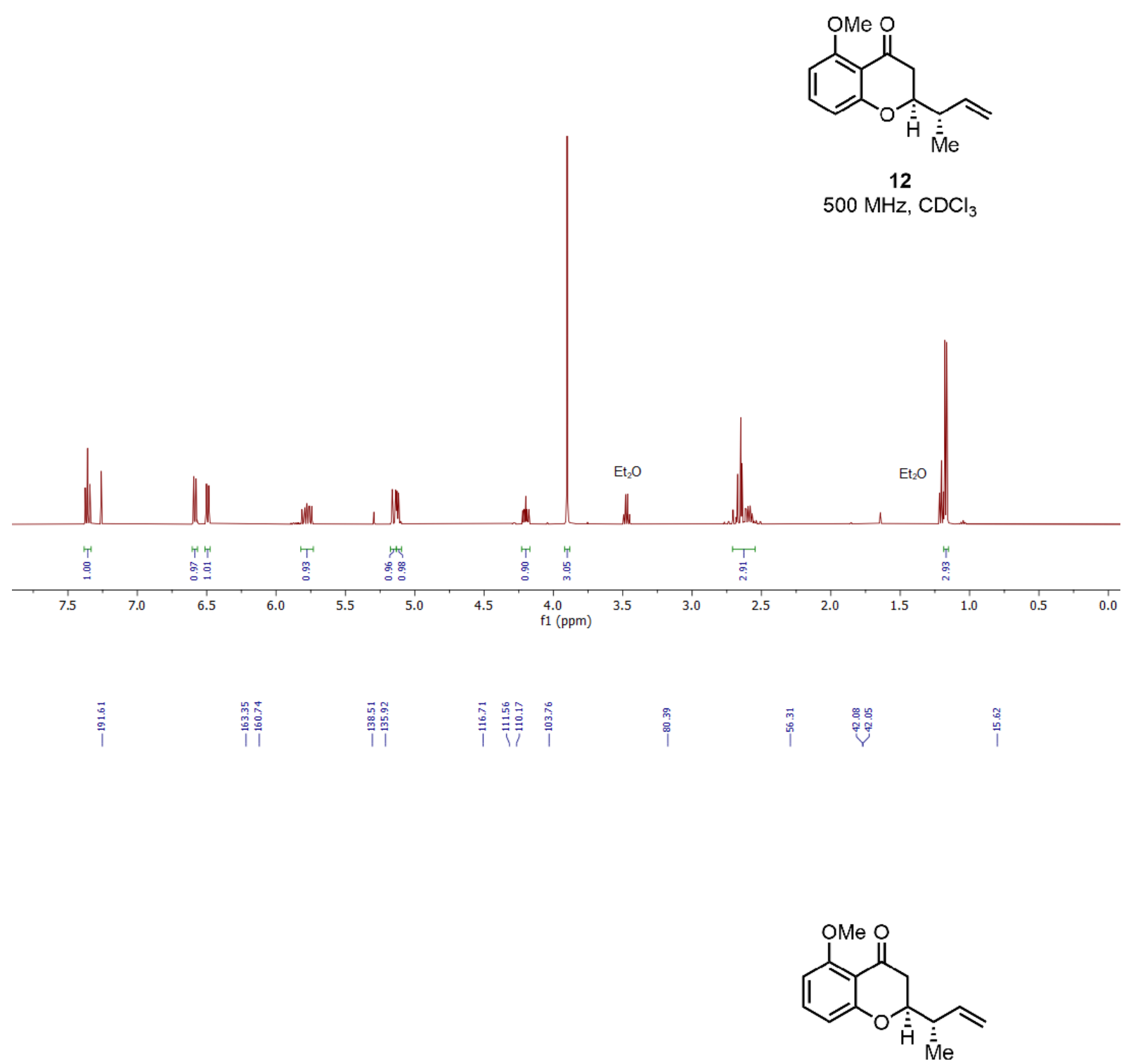

12

$126 \mathrm{MHz}, \mathrm{CDCl}_{3}$

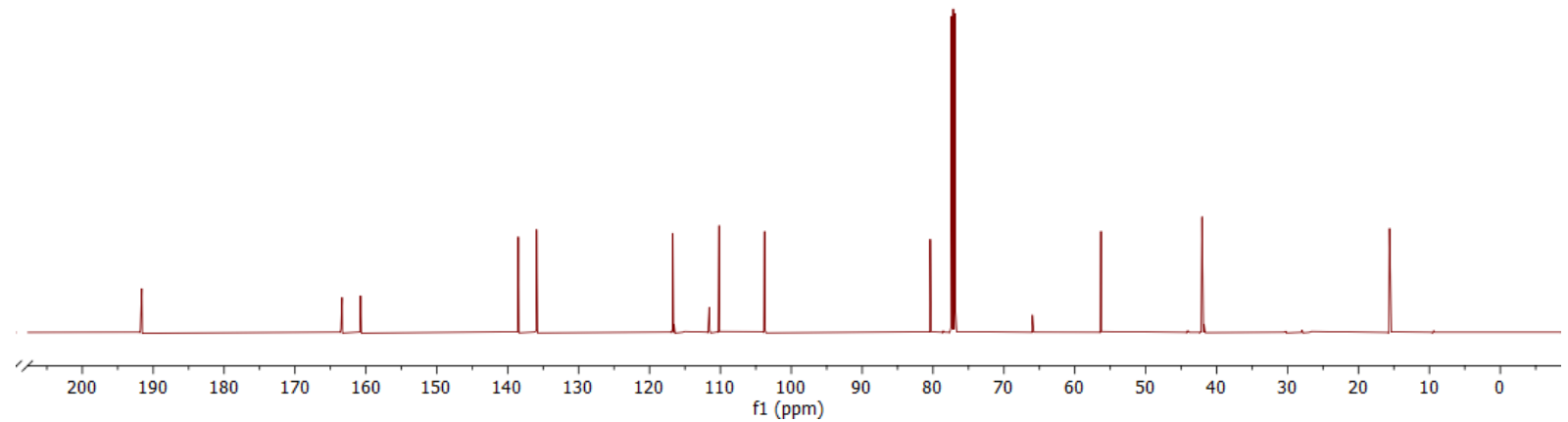




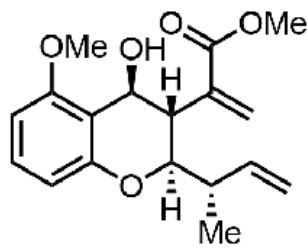

$26 a$

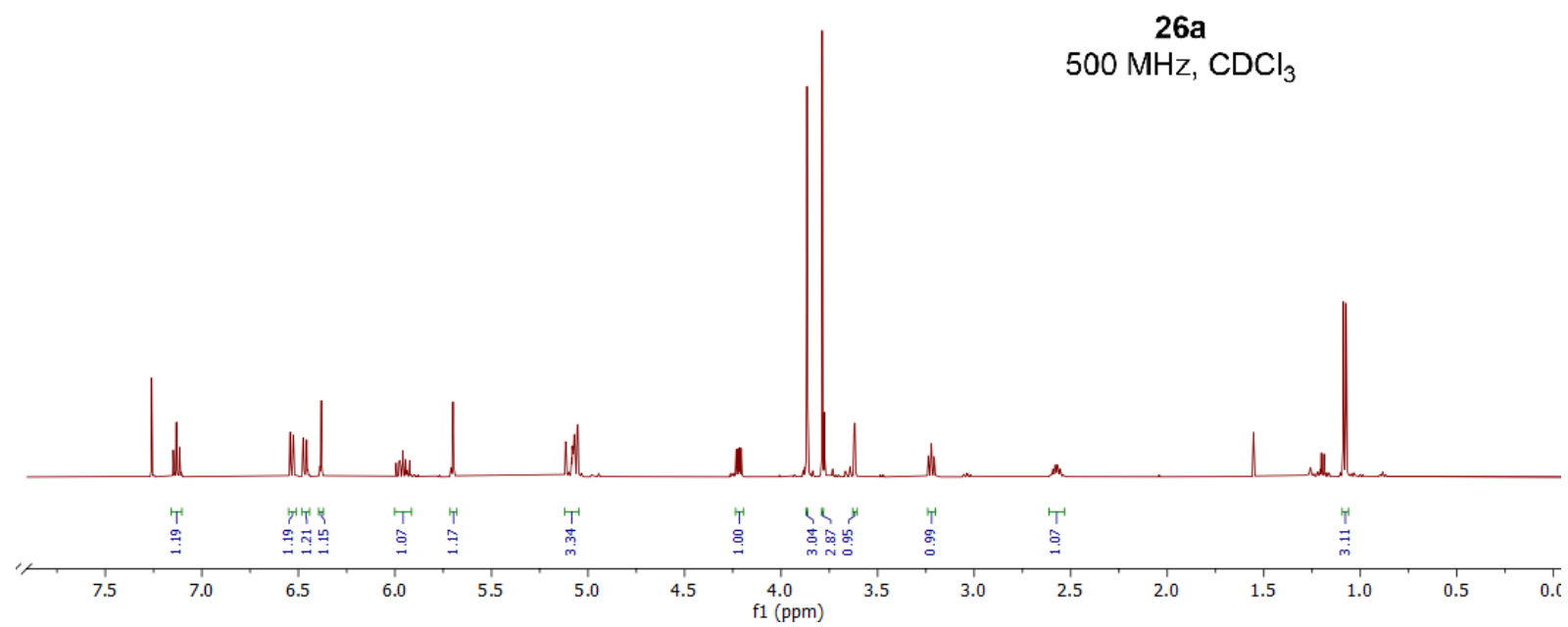

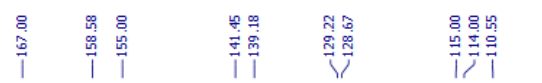

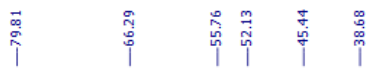

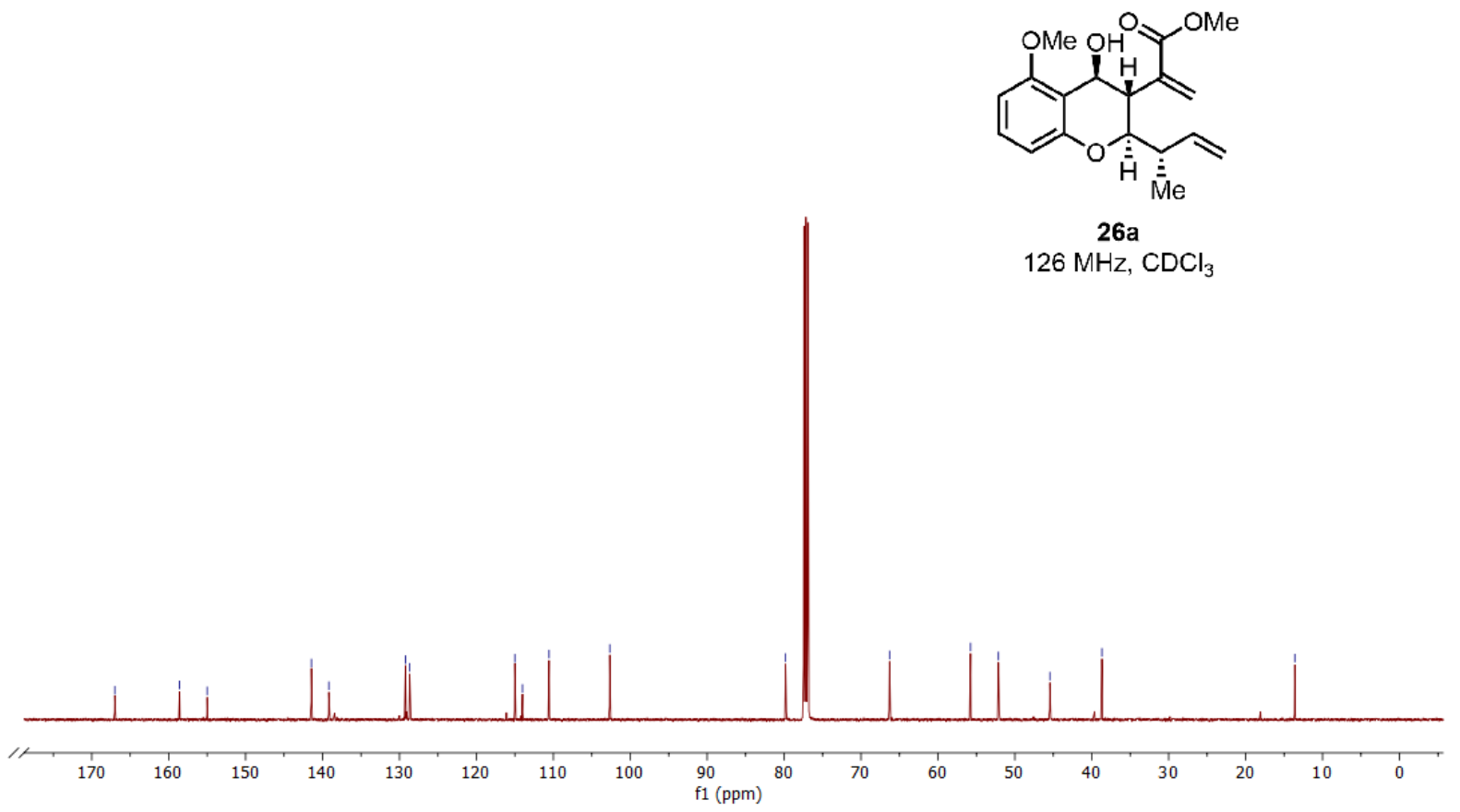




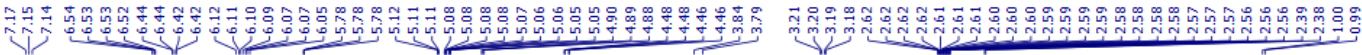

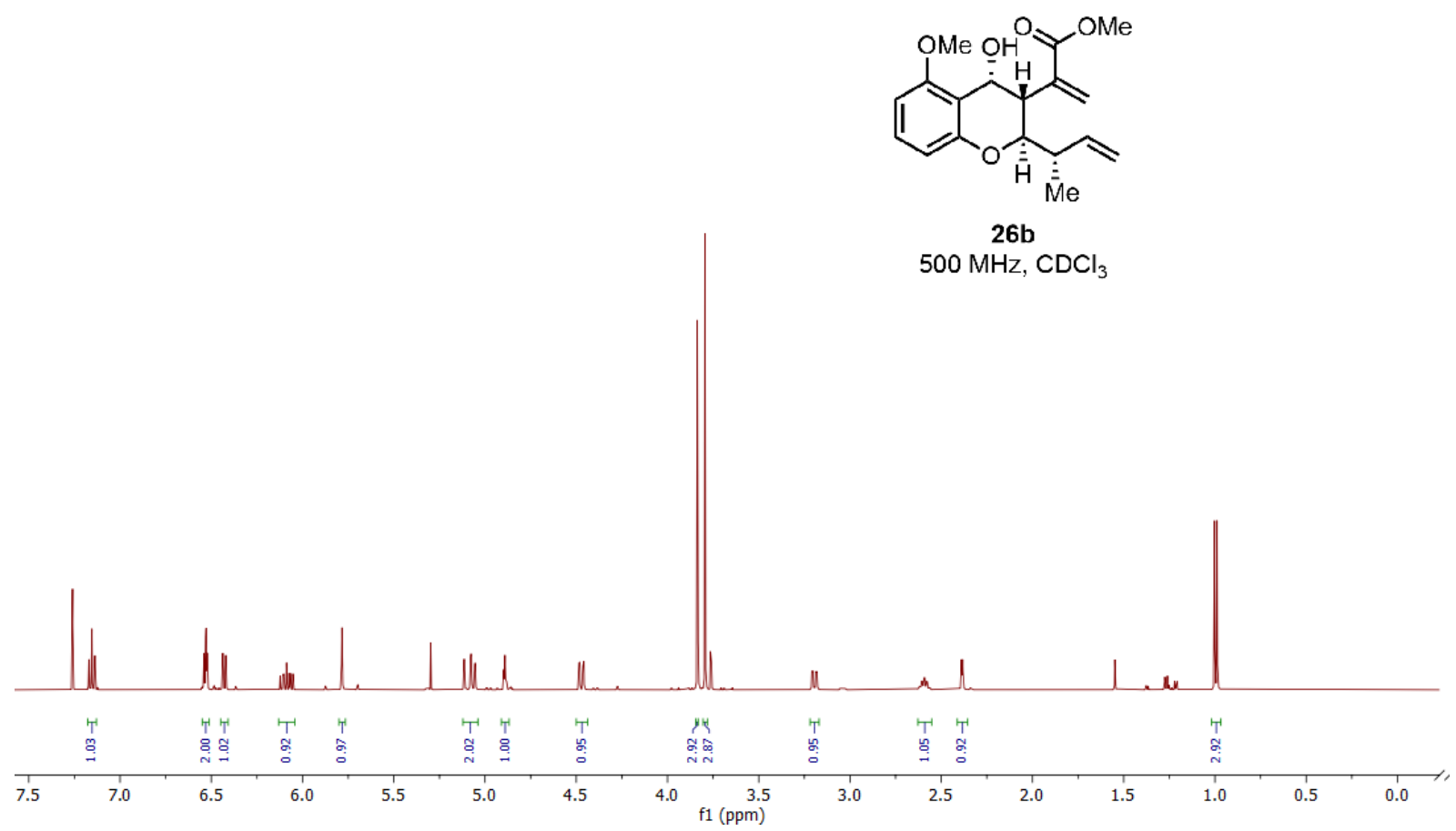

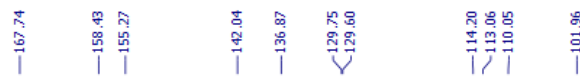

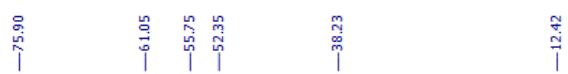

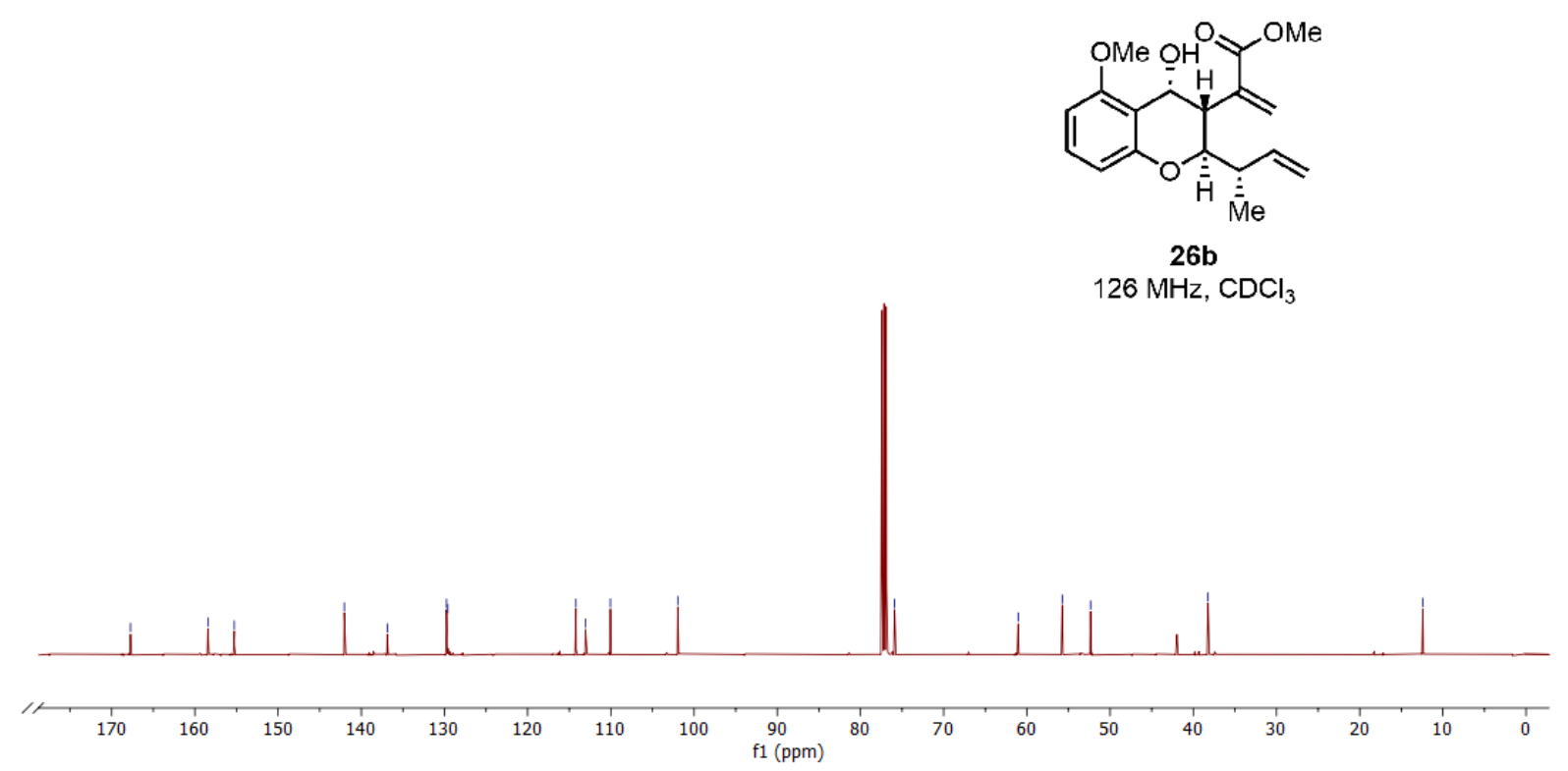




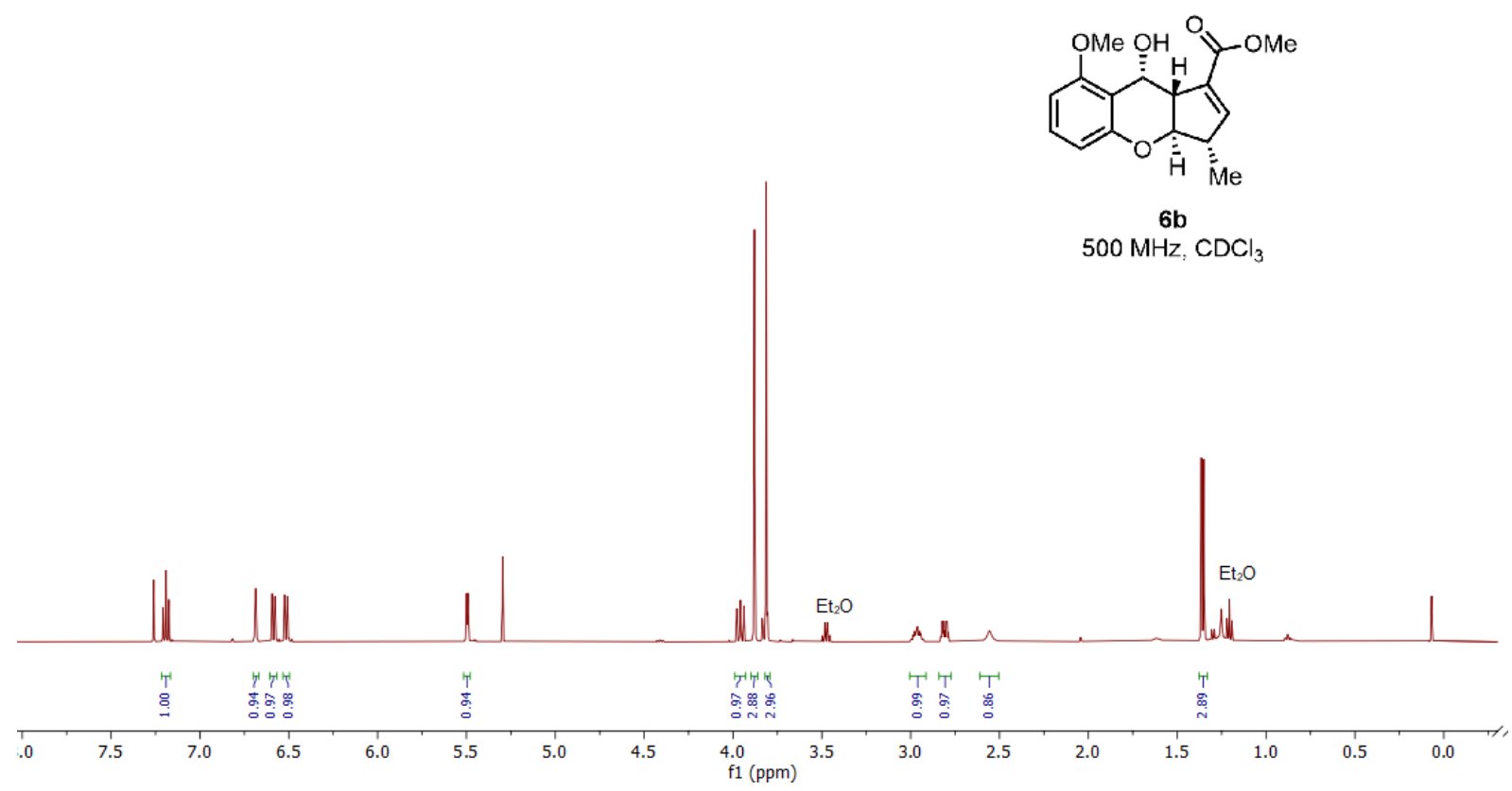

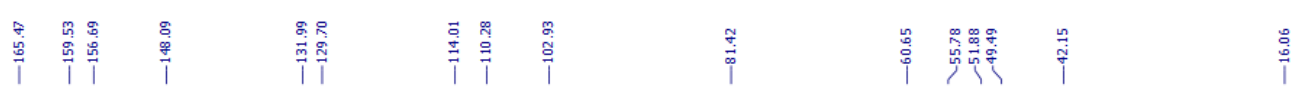

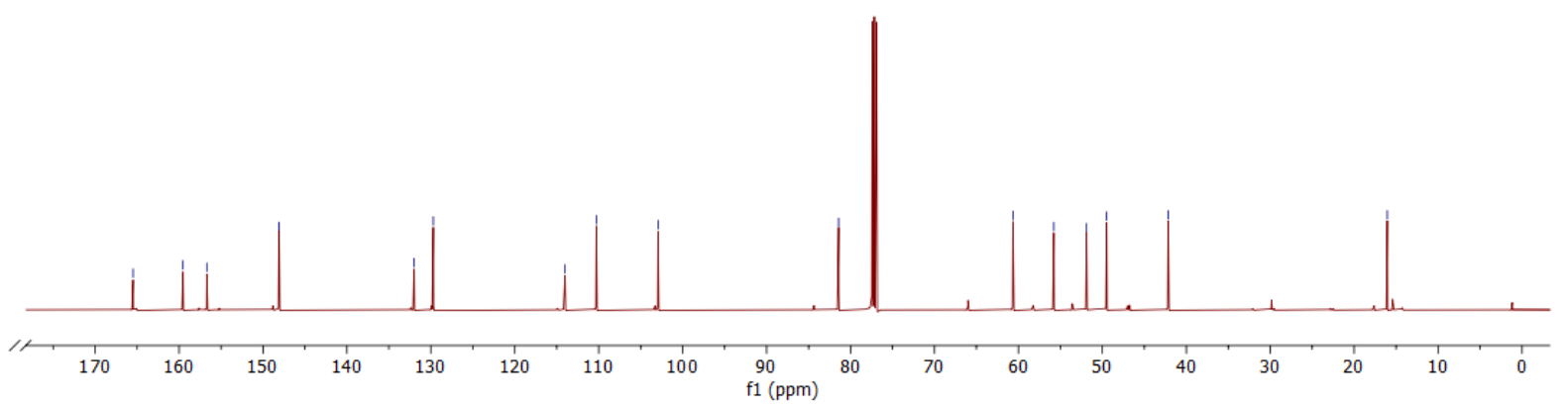




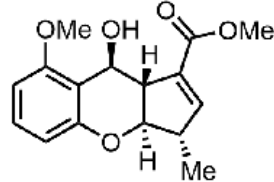

$6 a$

$500 \mathrm{MHz}, \mathrm{CDCl}_{3}$

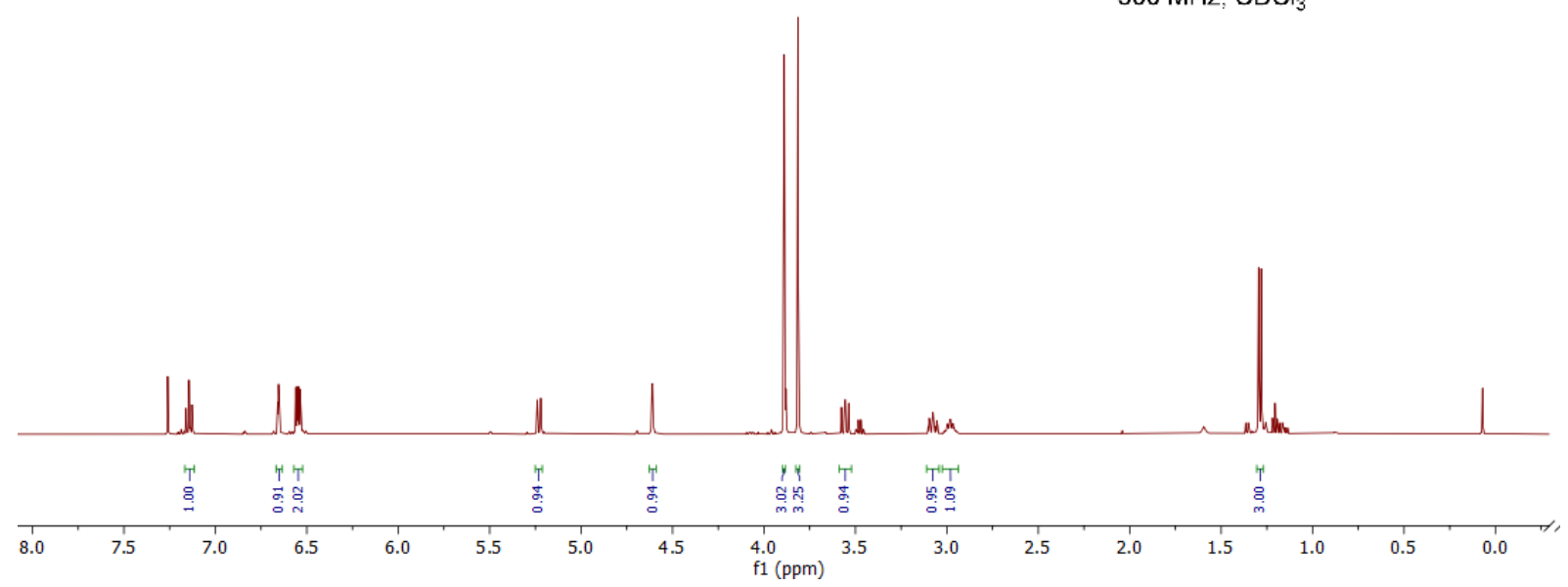

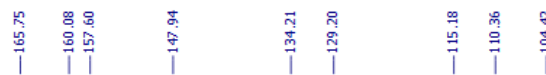

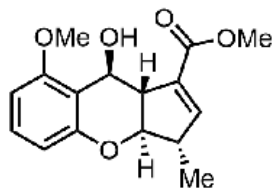

$6 a$

$126 \mathrm{MHz}^{\mathrm{CDCl}}$

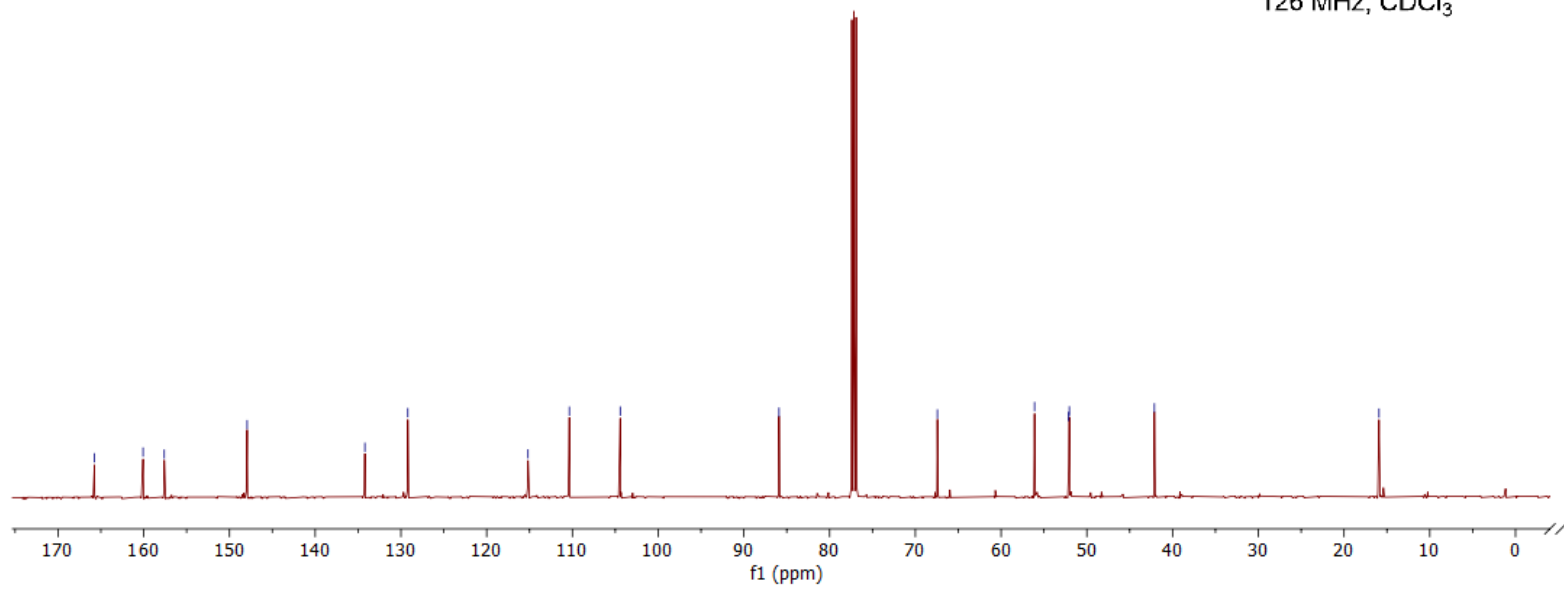




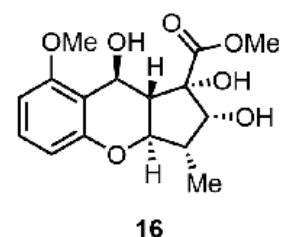

$500 \mathrm{MHz}, \mathrm{DMSO}-d_{6}$

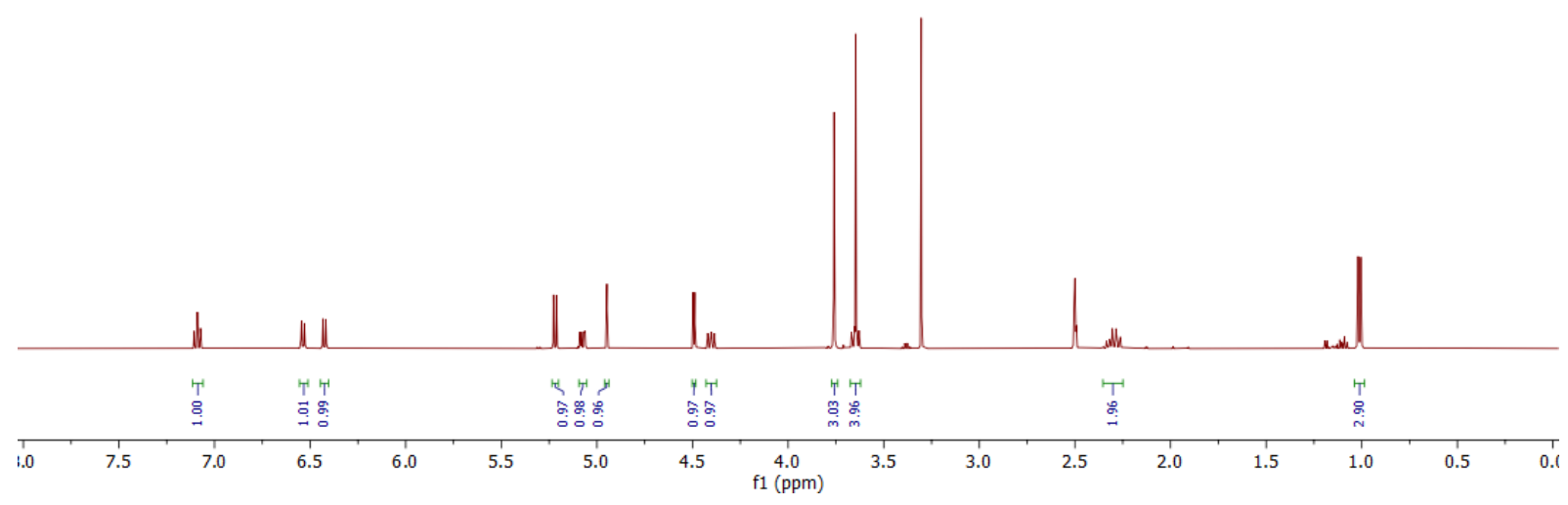

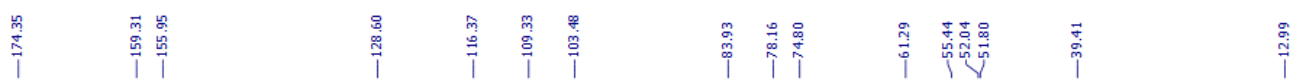

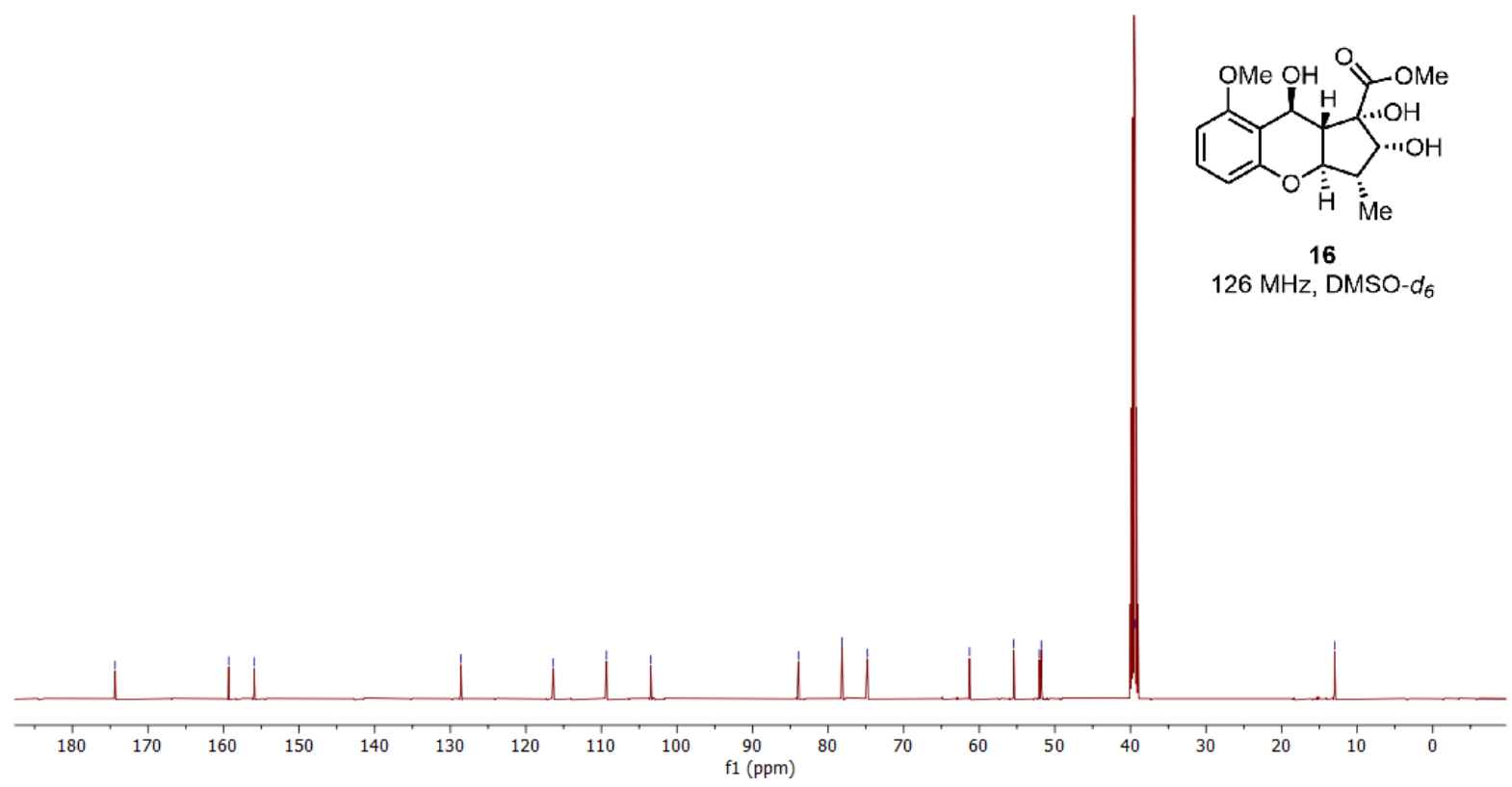




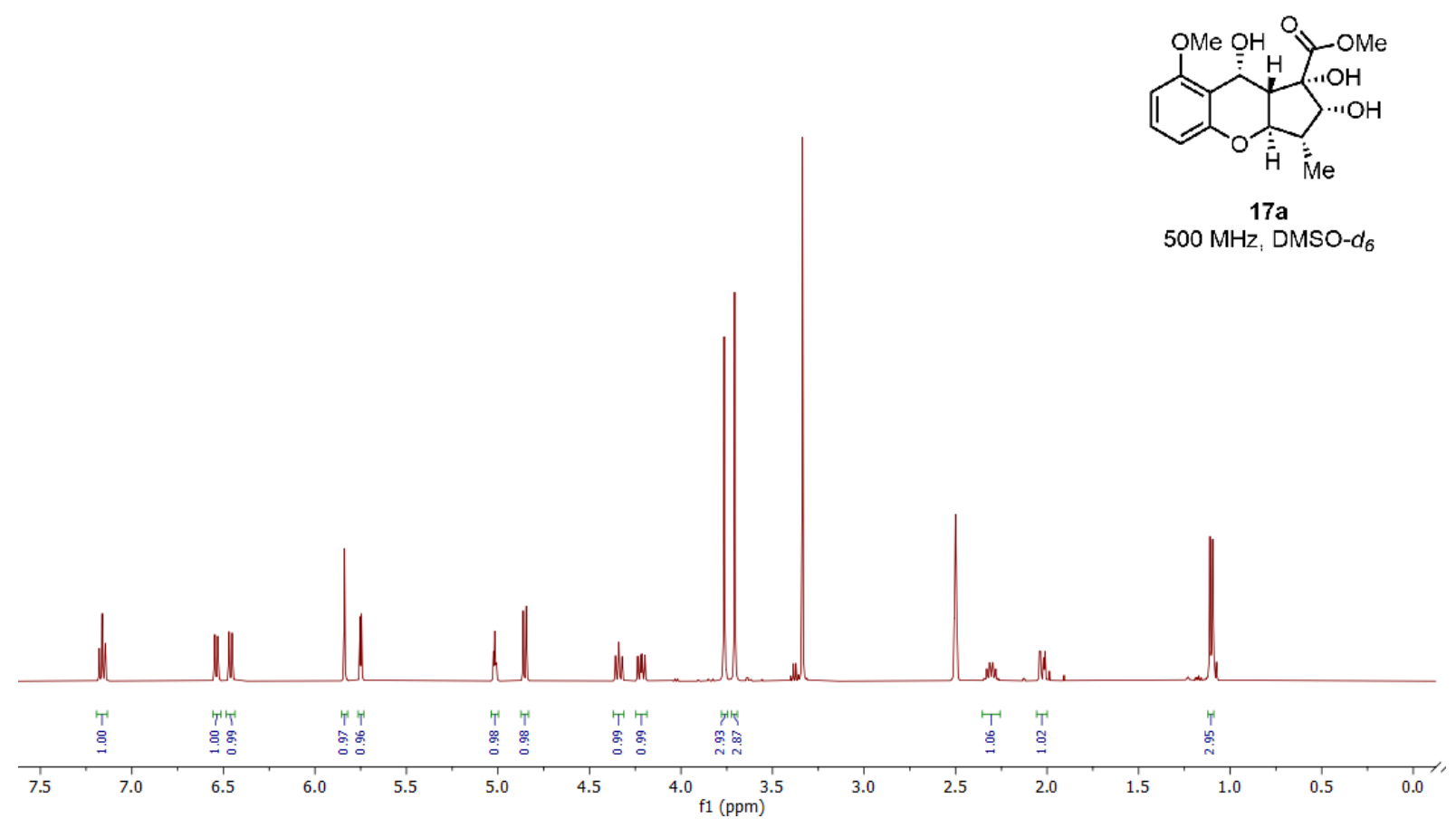

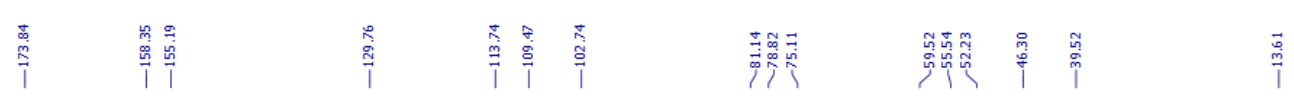

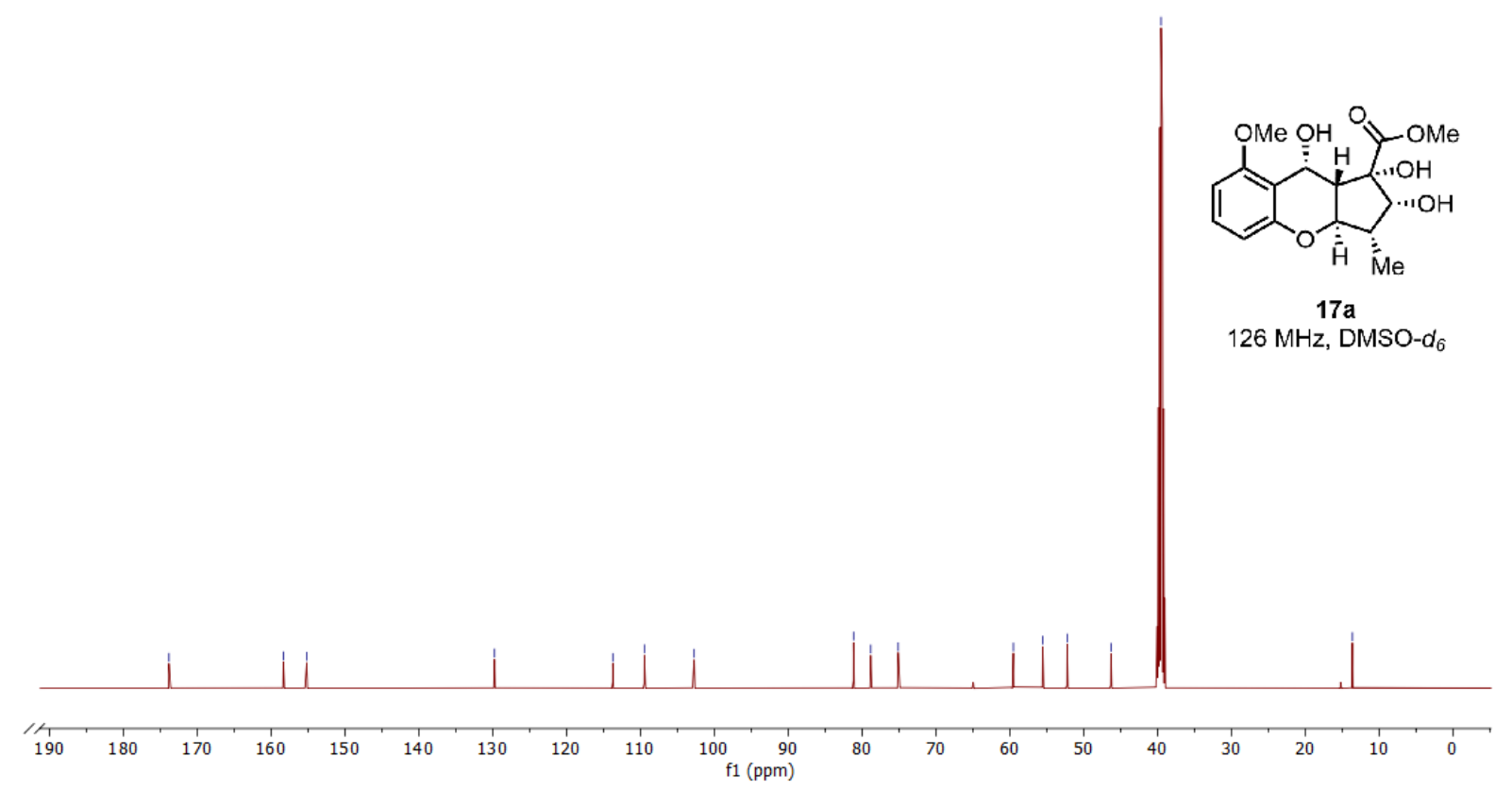




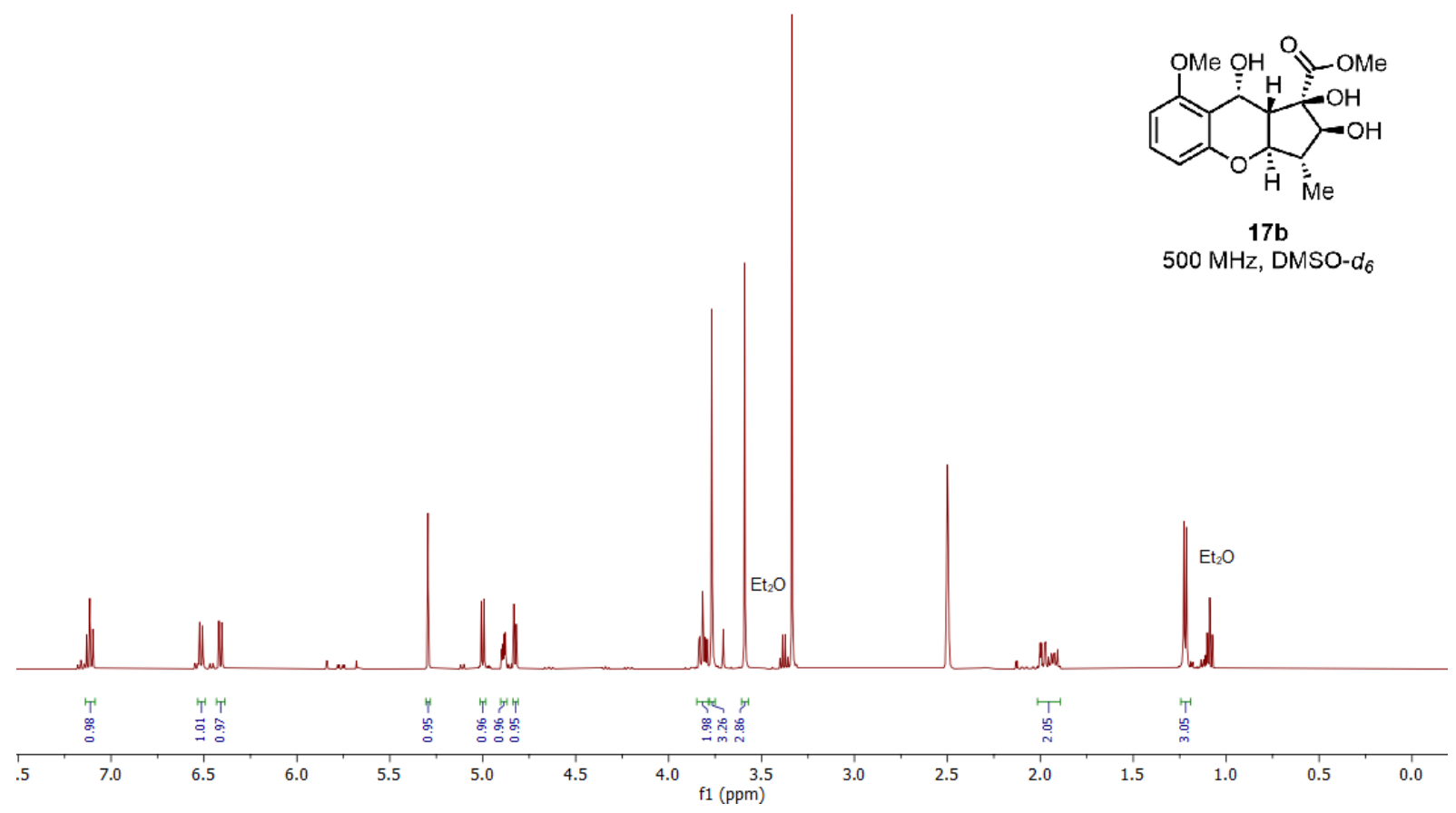

111

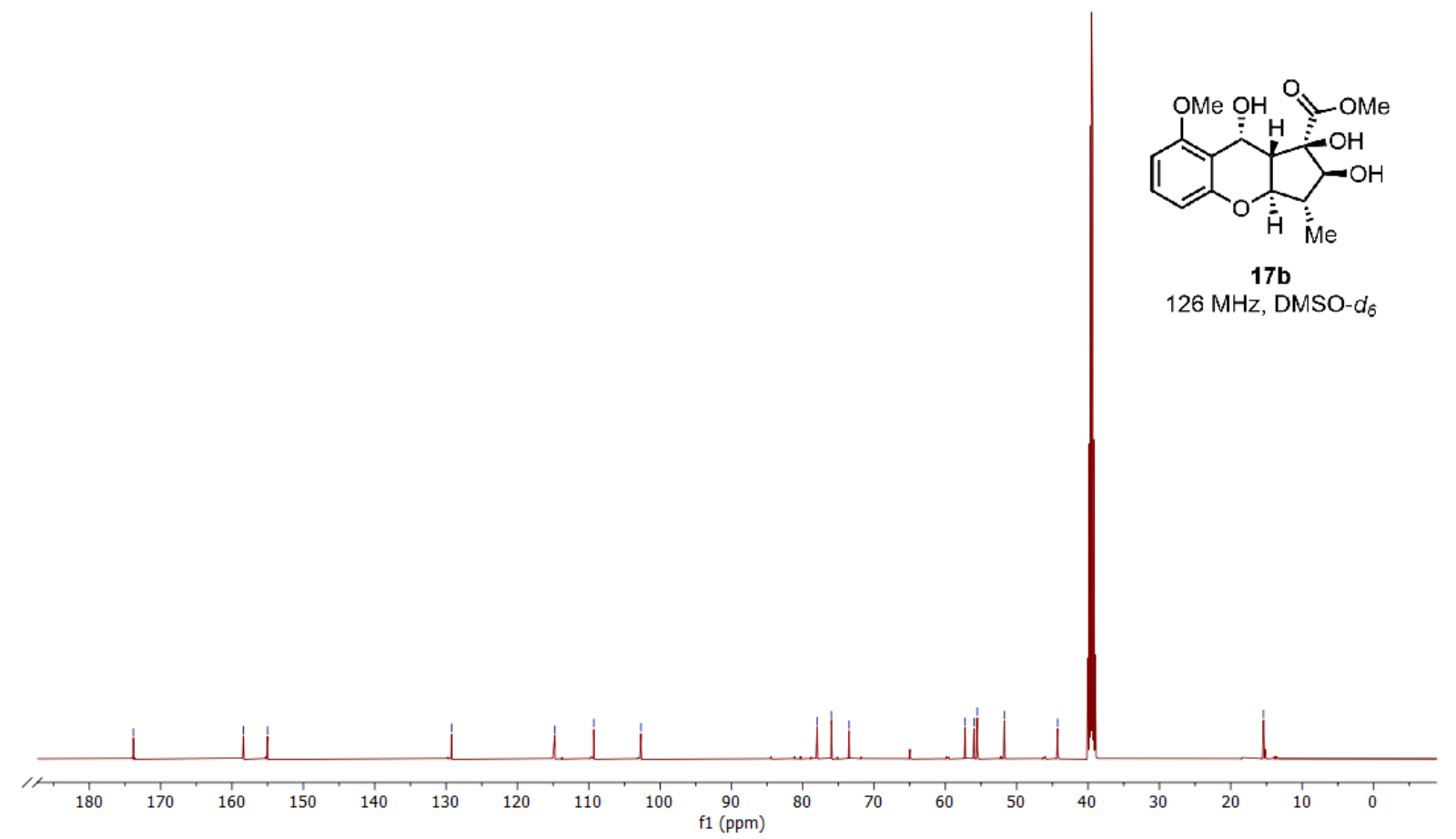




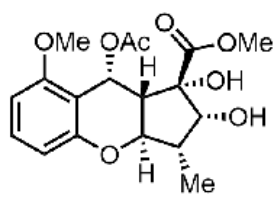

$18 \mathbf{a}$

$500 \mathrm{MHz}, \mathrm{CDCl}_{3}$

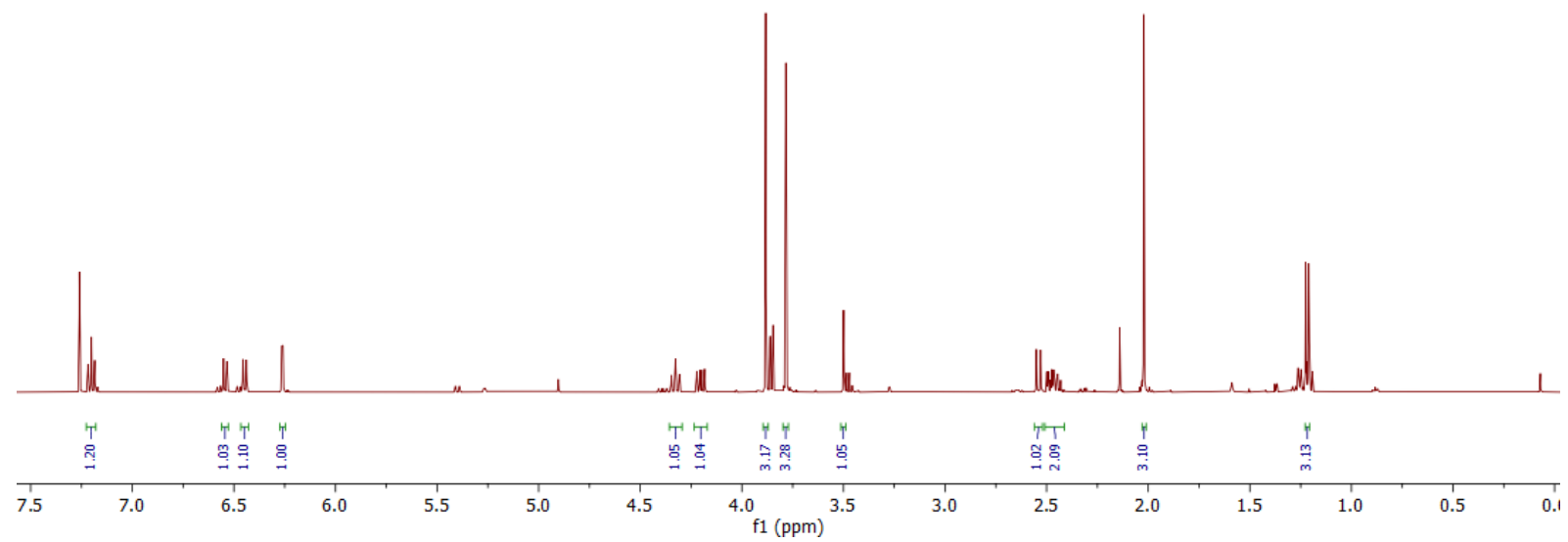

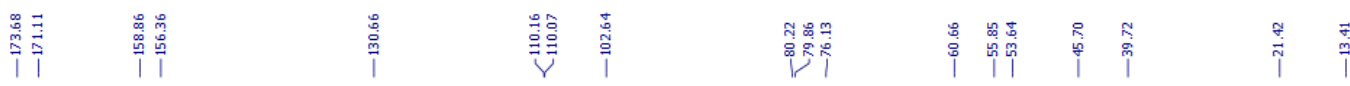

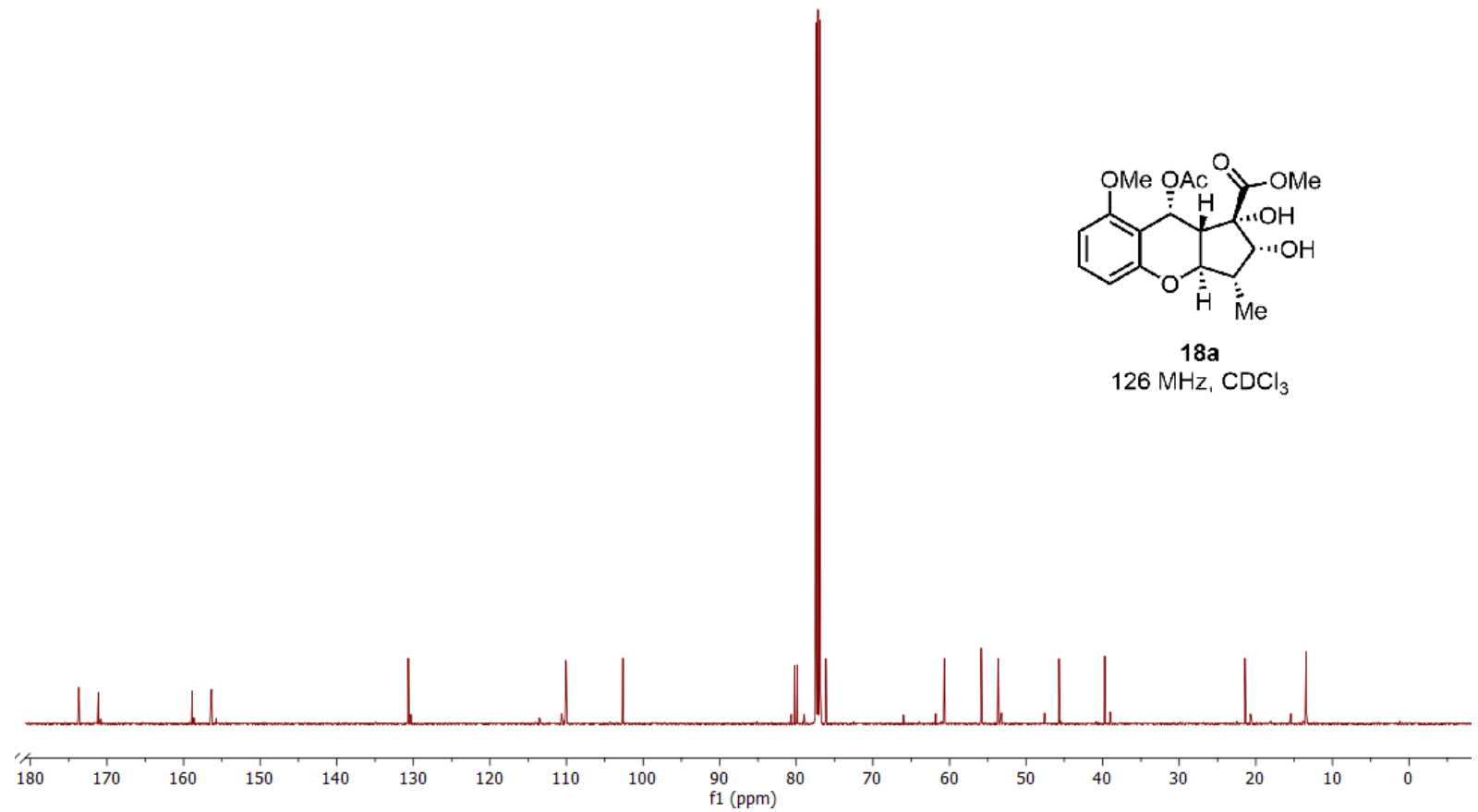




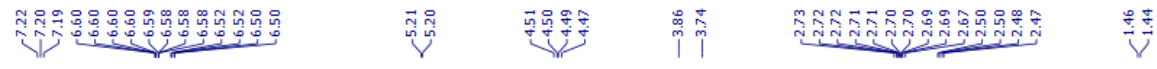
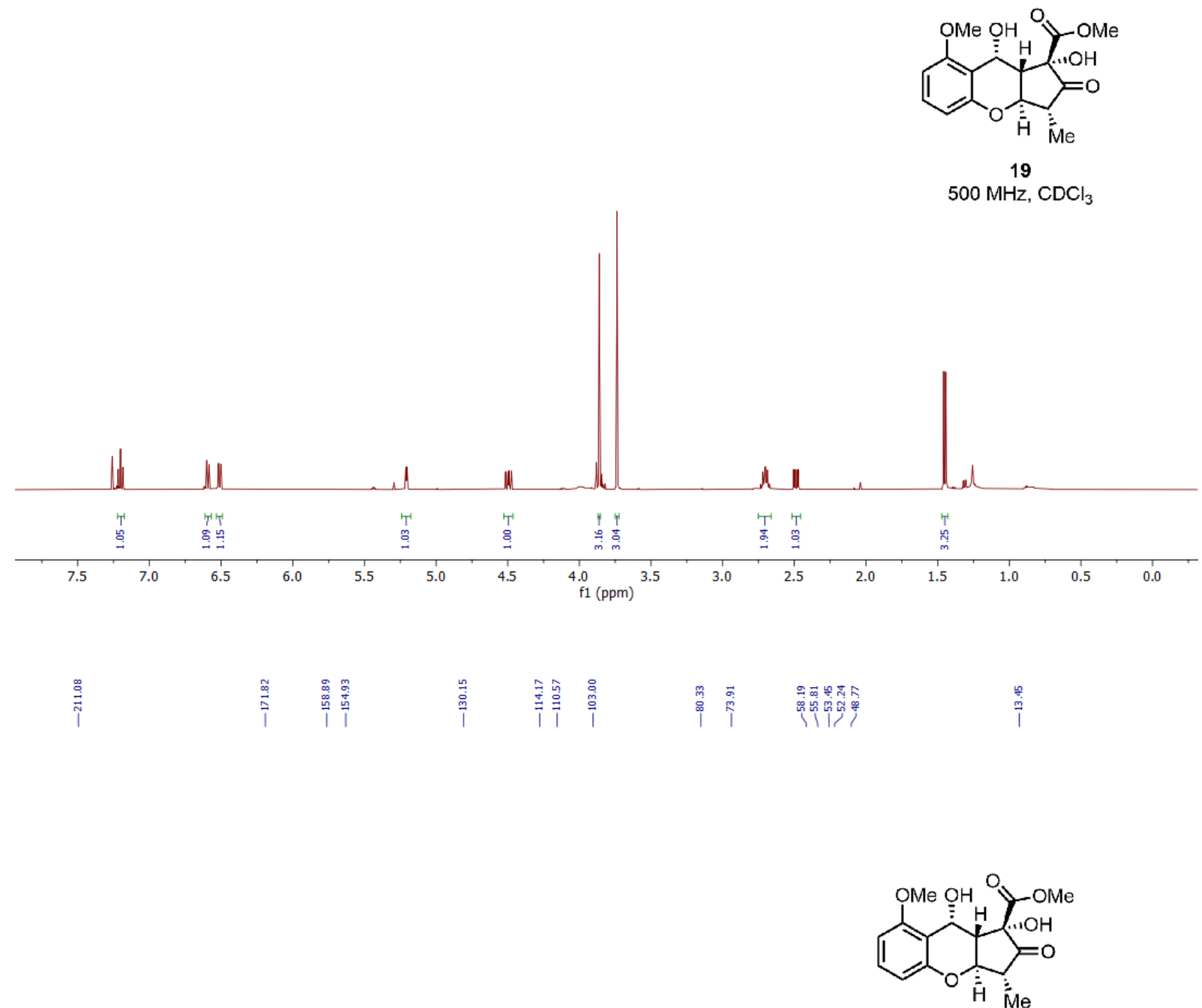

19

$126 \mathrm{MHz}, \mathrm{CDCl}_{3}$

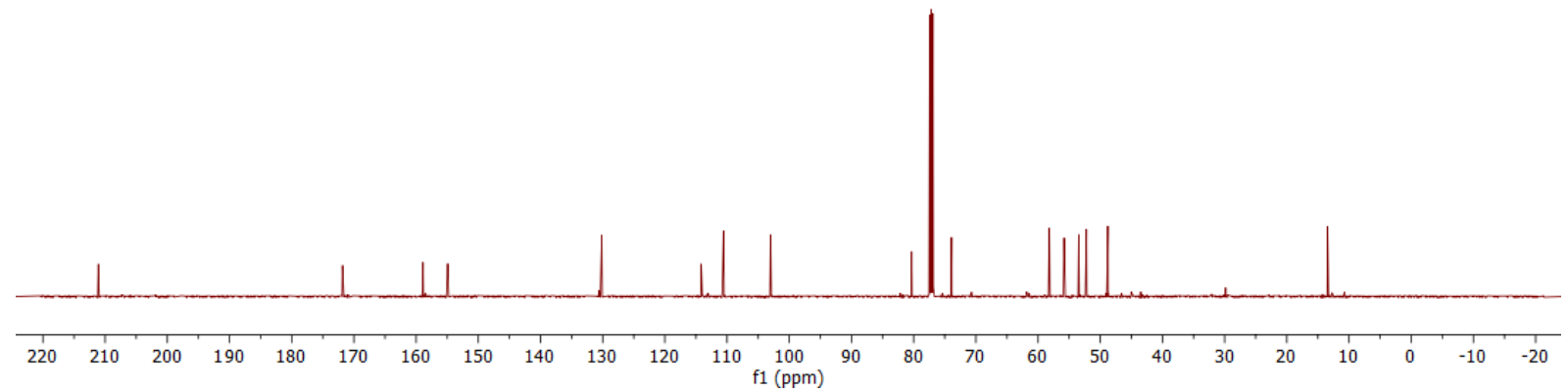




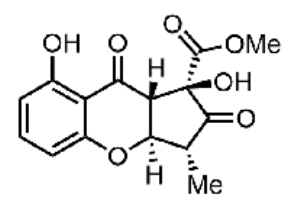

$500 \mathrm{MHz},{ }^{4}$ Acetone- $\mathrm{d}_{6}$
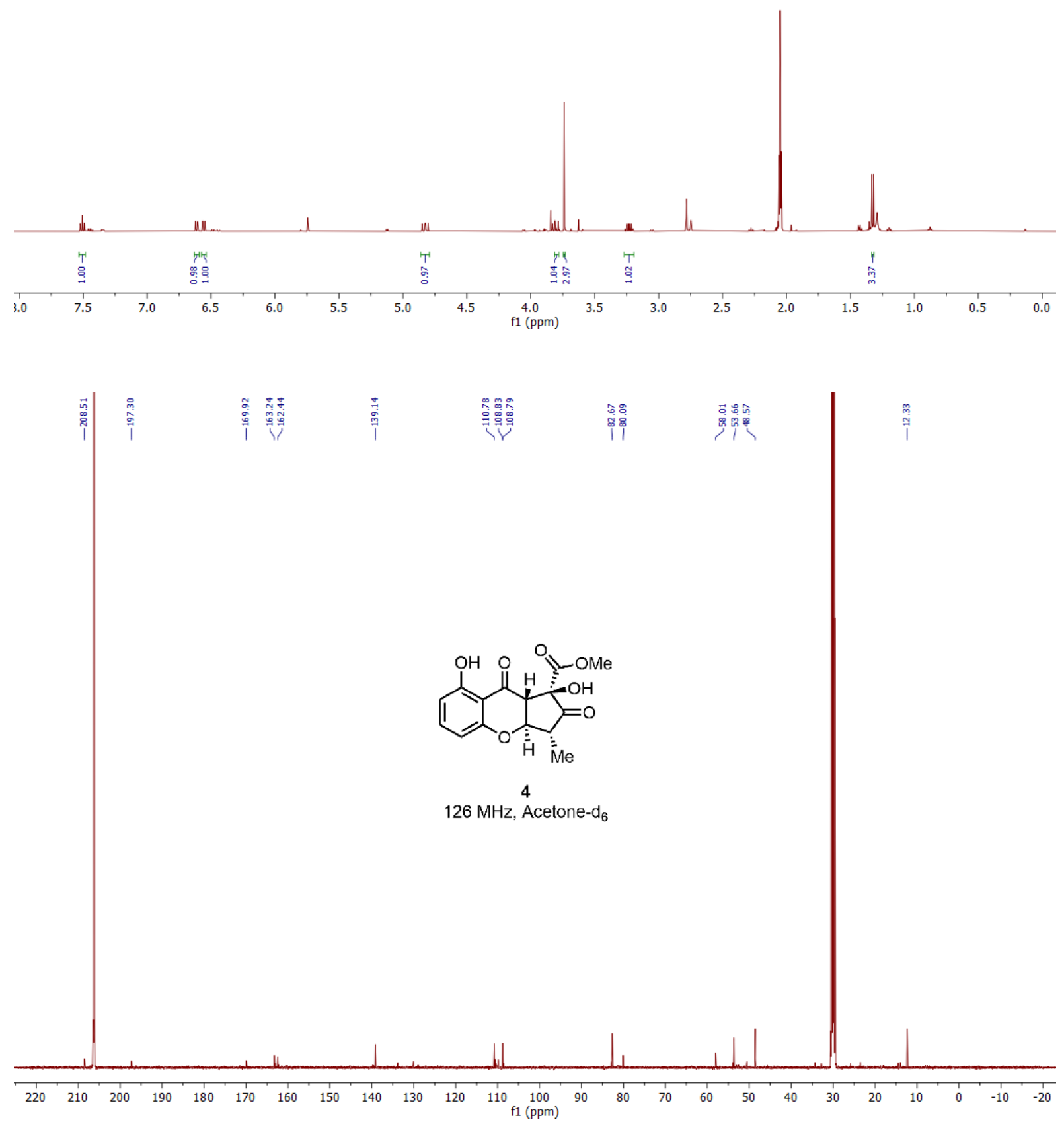

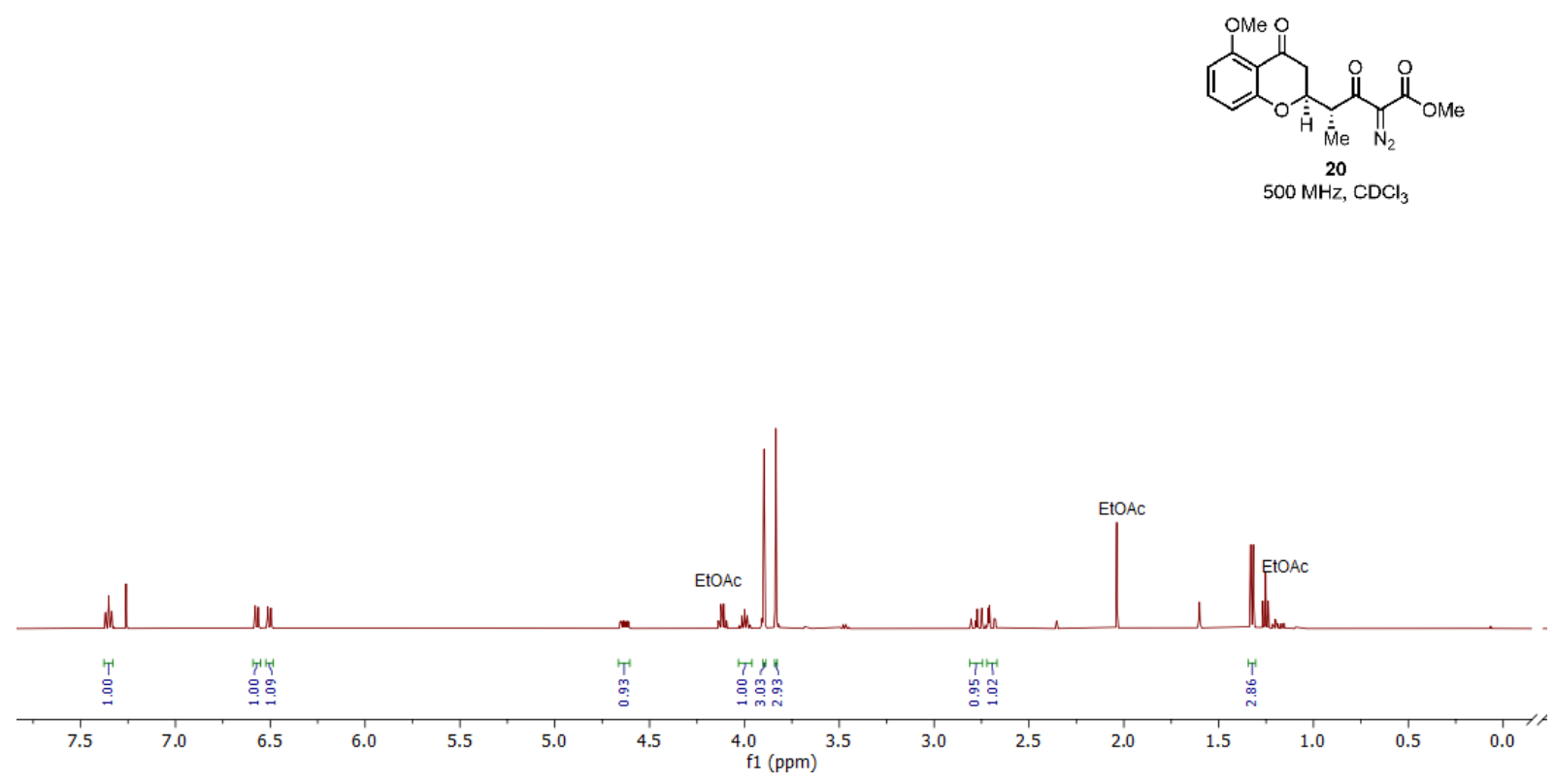

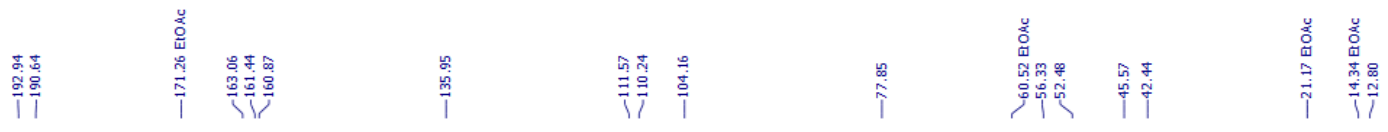

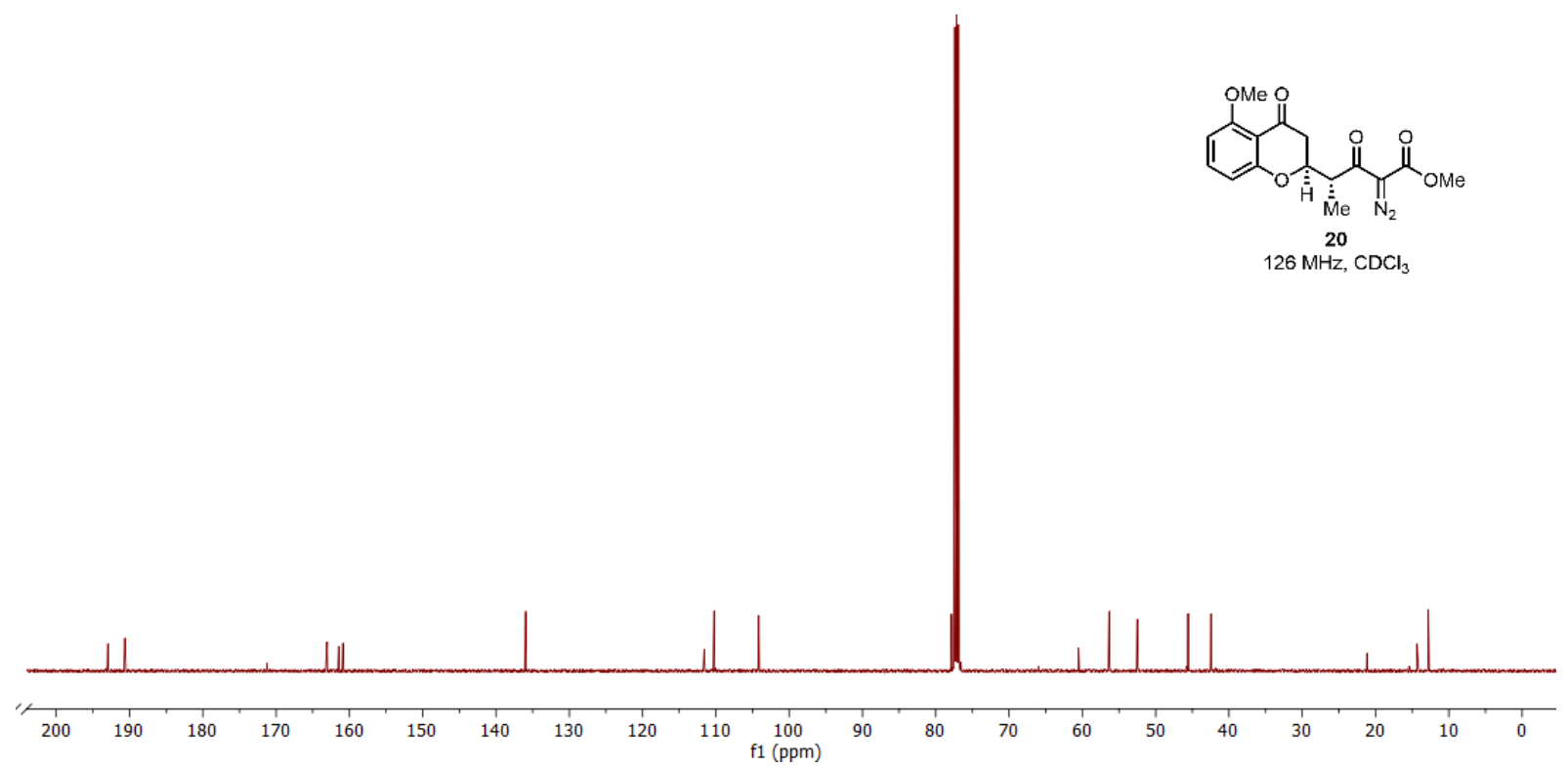



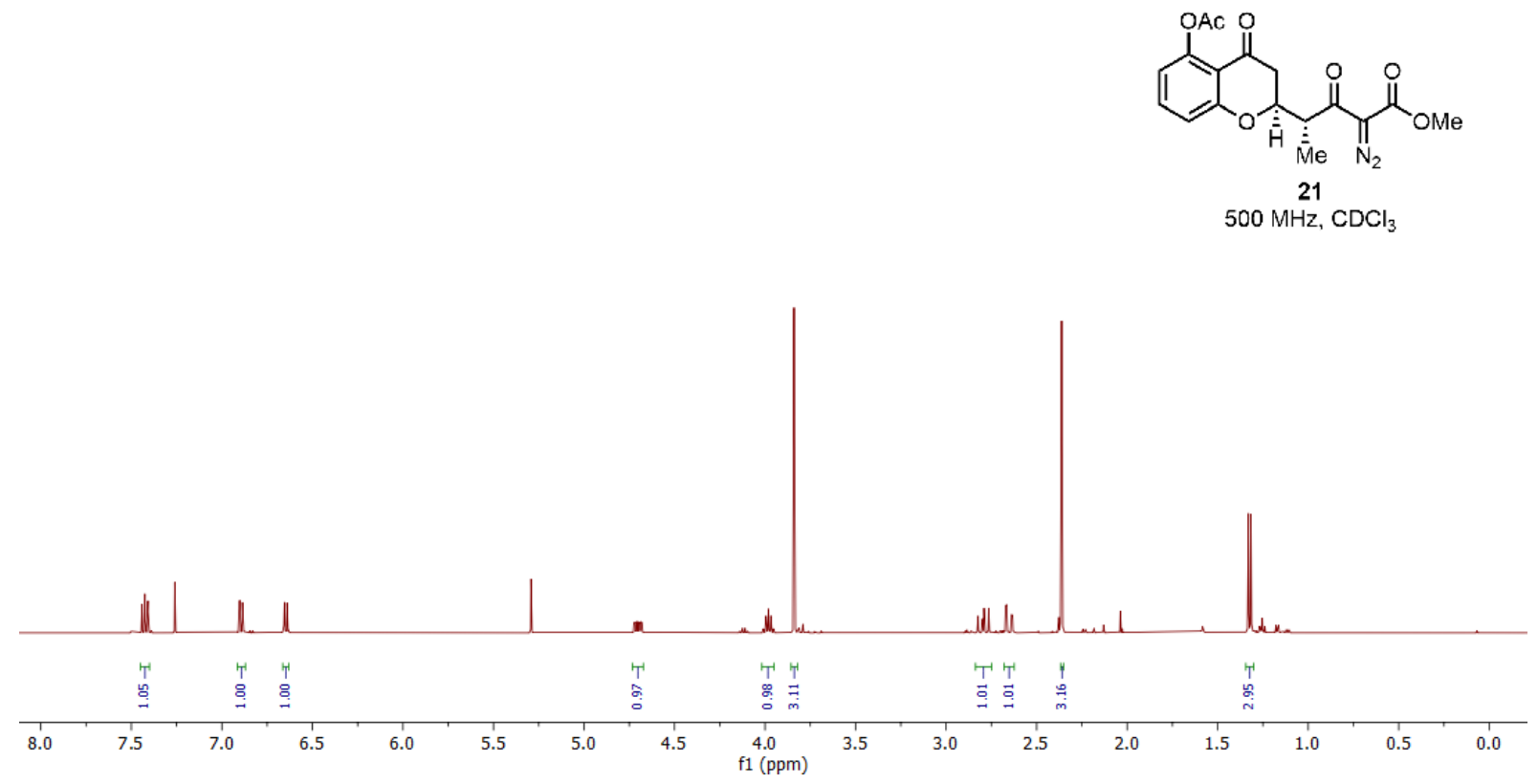

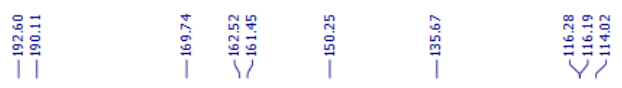

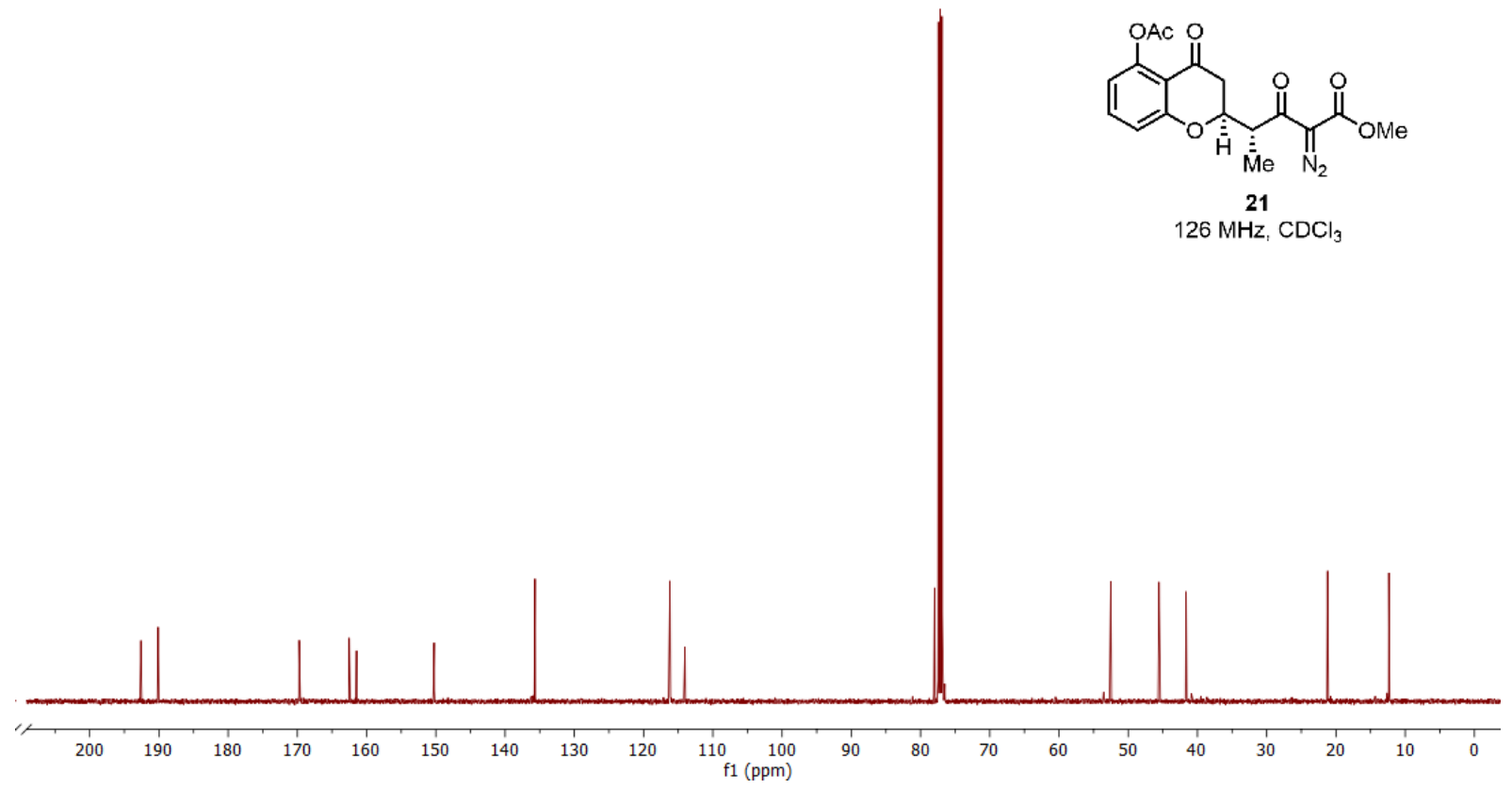




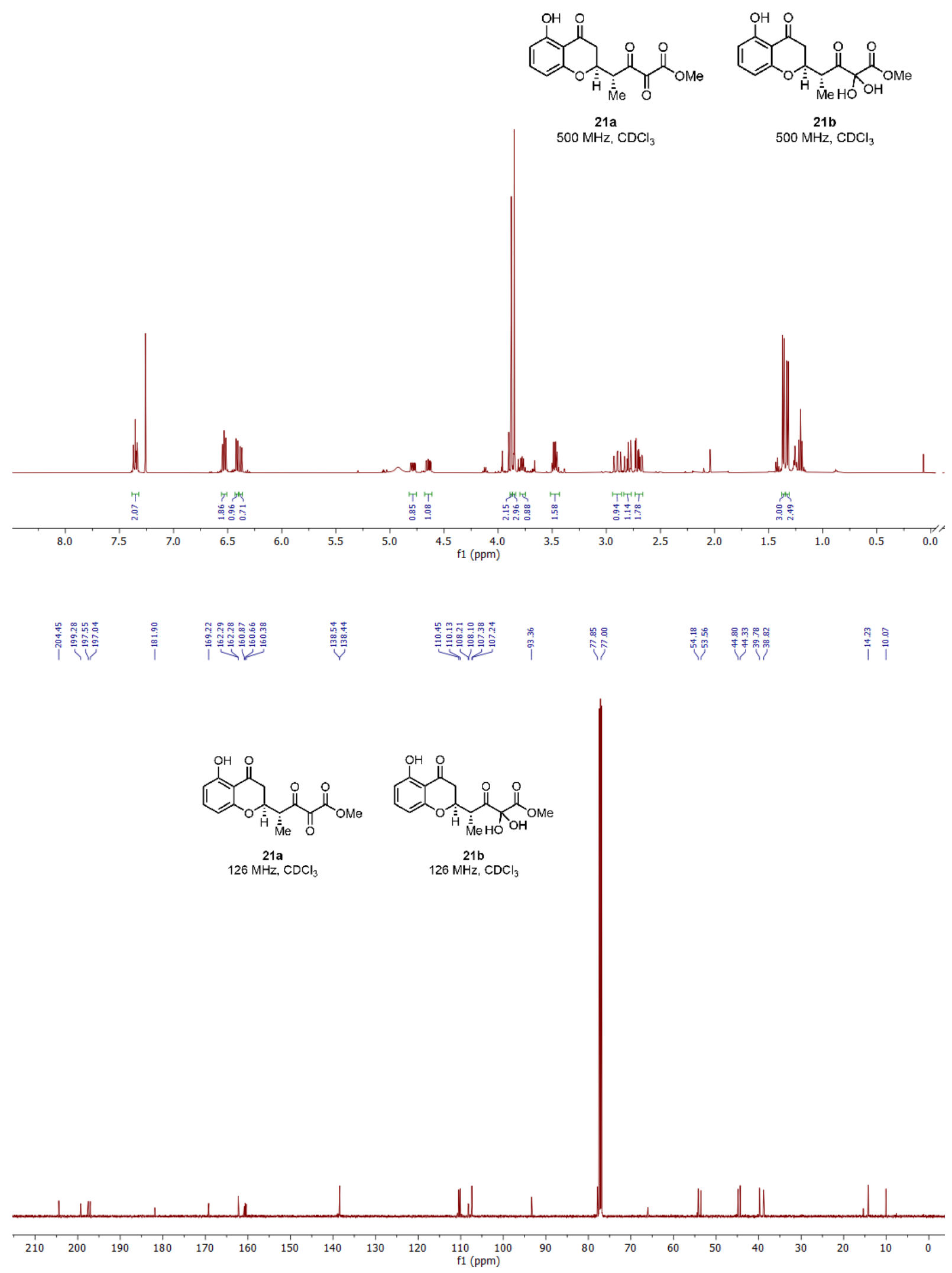




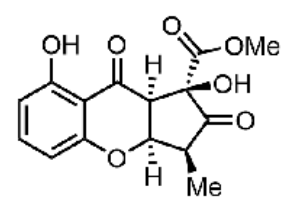

23

$500 \mathrm{MHz}$, acetone- $d_{6}$

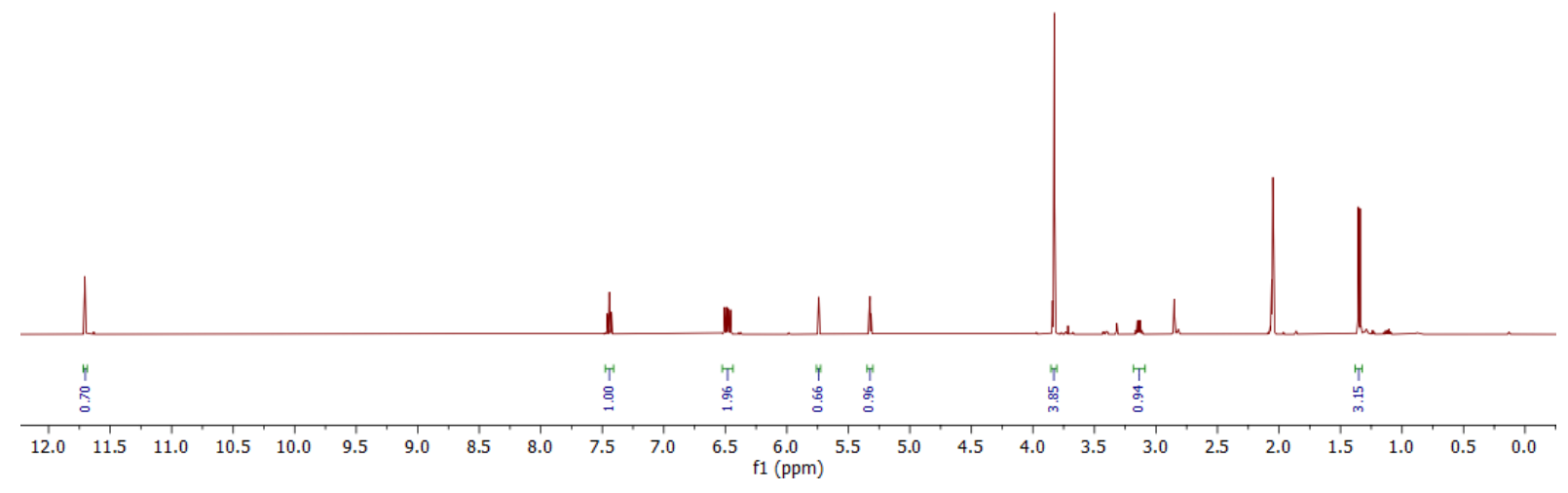

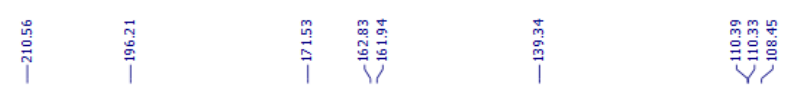

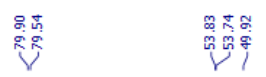

$\stackrel{\vec{p}}{i}$

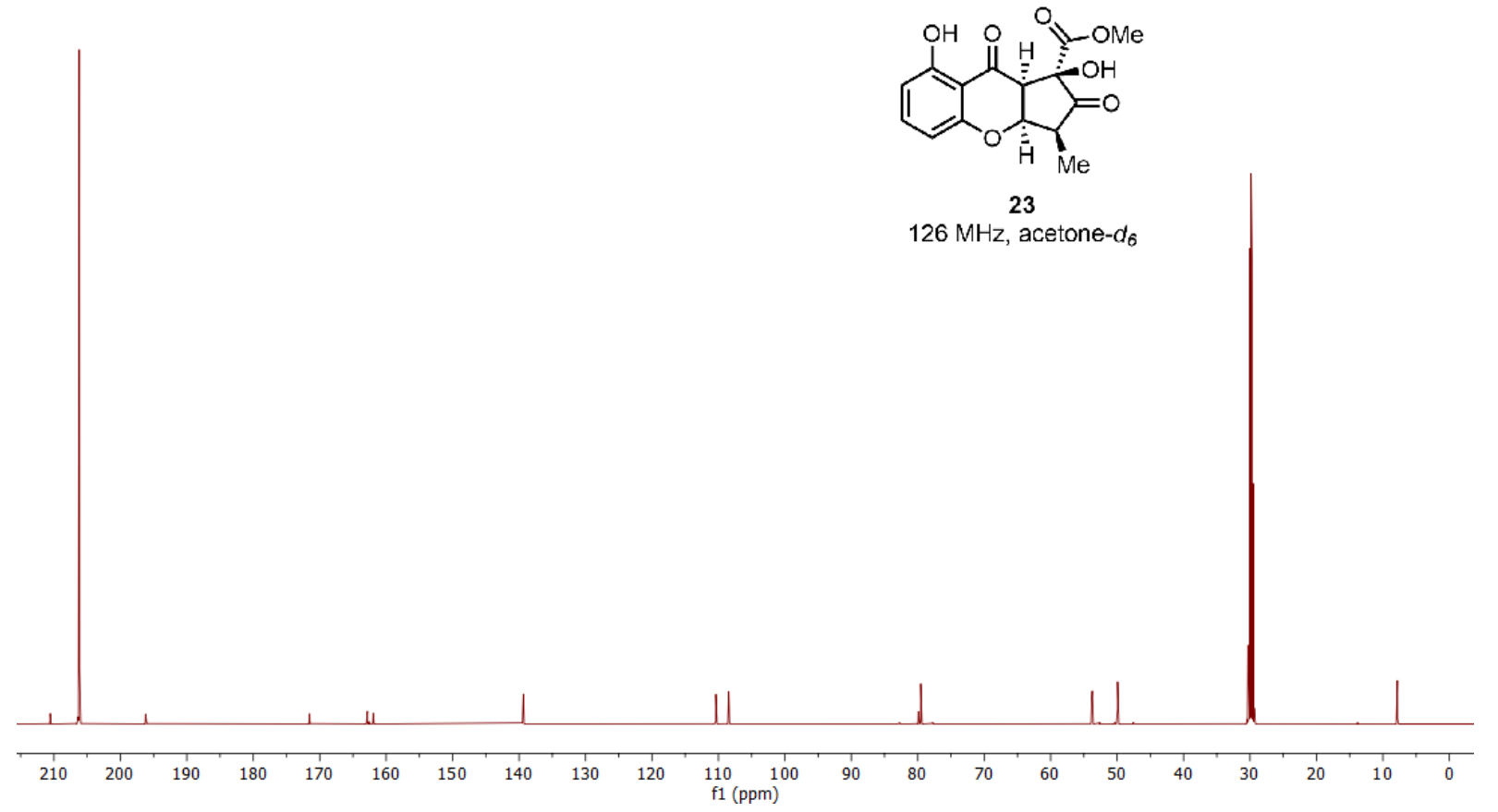



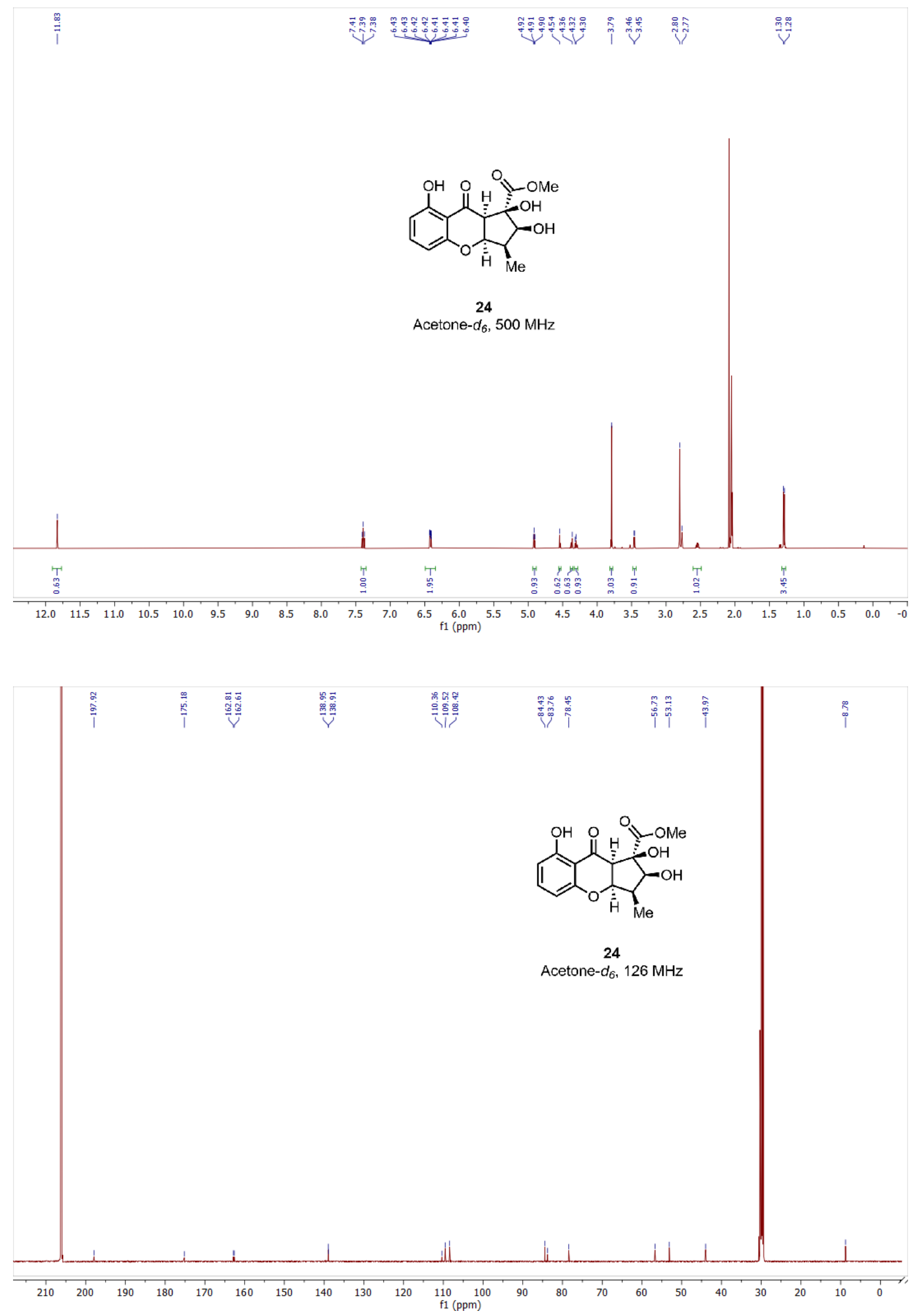


\section{X-Ray}

\subsection{Methyl $(R)-2-((2 R, 3 R)-2-((S)-b u t-3-e n-2-y l)-5-m e t h o x y-4-o x o c h r o m a n-3-y l)-$}

2-(phenylthio)propanoate (ent-15a)

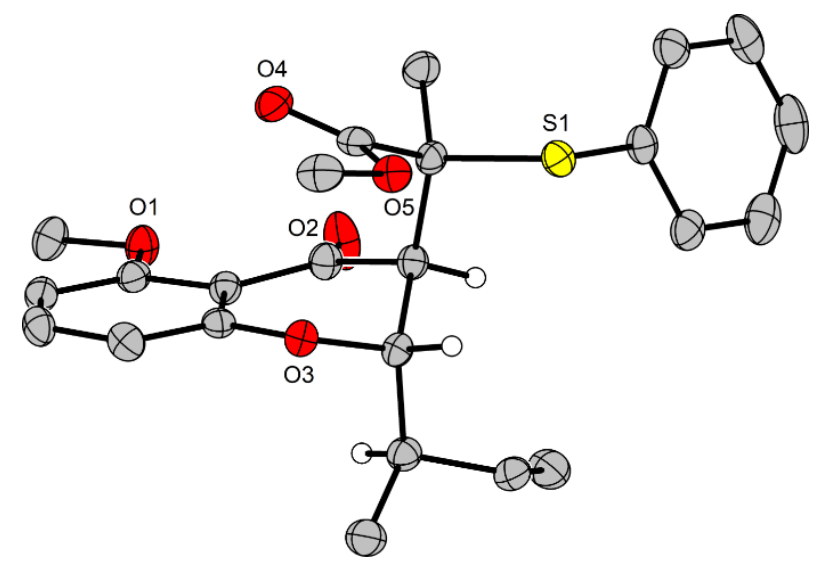

Figure 1. Crystal structure of ent-15a. Thermal ellipsoids are shown at 50\% probability.

A suitable crystal of $\mathrm{C}_{24} \mathrm{H}_{26} \mathrm{O}_{5} \mathrm{~S}$ was selected under inert oil and mounted using a MiTeGen loop. Intensity data of the crystal were recorded with a STADIVARI diffractometer (Stoe \& $\mathrm{Cie})$. The diffractometer was operated with $\mathrm{Cu}-\mathrm{K} \alpha$ radiation (1.54186 $\AA$, microfocus source) and equipped with a Dectris PILATUS 300K detector. Evaluation, integration and reduction of the diffraction data was carried out using the X-Area software suite. ${ }^{[4]}$ Multi-scan and numerical absorption corrections were applied with the LANA and X-RED32 modules of the X-Area software suite. ${ }^{[5,6]}$ The structure was solved using dual-space methods (SHELXT-2014/5) and refined against $F^{2}$ (SHELXL-2018/3 using ShelXle interface). ${ }^{[7-9]}$ All non-hydrogen atoms were refined with anisotropic displacement parameters. The hydrogen atoms were refined using the "riding model" approach with isotropic displacement parameters 1.2 times (1.5 times for the methyl groups) of that of the preceding carbon atom. CCDC 2129123 contains the supplementary crystallographic data for this paper. These data can be obtained free of charge from The Cambridge Crystallographic Data Centre via www.ccdc.cam.ac.uk/structures.

Empirical formula

Molar mass / $\mathrm{g} \cdot \mathrm{mol}^{-1}$

Space group (No.)

$a / \AA$

b / $\AA$

$c / \AA$

$\beta /^{\circ}$

$V / \AA^{3}$

$$
\begin{gathered}
\mathrm{C}_{24} \mathrm{H}_{26} \mathrm{O}_{5} \mathrm{~S} \\
426.51 \\
P 2_{1}(4) \\
7.54890(10) \\
18.3791(2) \\
7.77100(10) \\
99.1250(10) \\
1064.52(2)
\end{gathered}
$$


Z

$\rho_{\text {calc. }} / \mathrm{g} \cdot \mathrm{cm}^{-3}$

$\mu / \mathrm{mm}^{-1}$

Color

Crystal habitus

Crystal size $/ \mathrm{mm}^{3}$

$T / \mathrm{K}$

$\lambda / \AA$

$\theta$ range $I^{\circ}$

Range of Miller indices

Absorption correction

$T_{\min }, T_{\max }$

$R_{\text {int }}, R_{\sigma}$

Completeness of the data set

No. of measured reflections

No. of independent reflections

No. of parameters

No. of restraints

$S$ (all data)

$R(F)(I \geq 2 \sigma(I)$, all data $)$

$w R\left(F^{2}\right)(I \geq 2 \sigma(I)$, all data $)$

Extinction coefficient

Flack parameter $x$

$\Delta \rho_{\max }, \Delta \rho_{\min } / \mathrm{e} \cdot \AA^{-3}$
1.331

1.628

colorless

block

$0.237 \times 0.139 \times 0.127$

100

$1.54186\left(\mathrm{Cu}-\mathrm{K}_{\alpha}\right)$

4.812 to 76.278

$-9 \leq h \leq 9$

$-22 \leq k \leq 23$

$-4 \leq l \leq 9$

multi-scan and numerical

$0.3710,0.8102$

$0.0207,0.0140$

0.996

24859

4336

276

1

1.059

$0.0234,0.0238$

$0.0606,0.0608$

$0.0027(5)$

$-0.008(5)$

$0.180,-0.143$ 


\subsection{Methyl $(1 S, 2 R, 3 S, 3 \mathrm{a} R, 9 S, 9 \mathrm{a} S)-1,2,9$-trihydroxy-8-methoxy-3-methyl-1,2,3,3a,9,9a-}

hexahydrocyclopenta[b]chromene-1-carboxylate (ent-16)

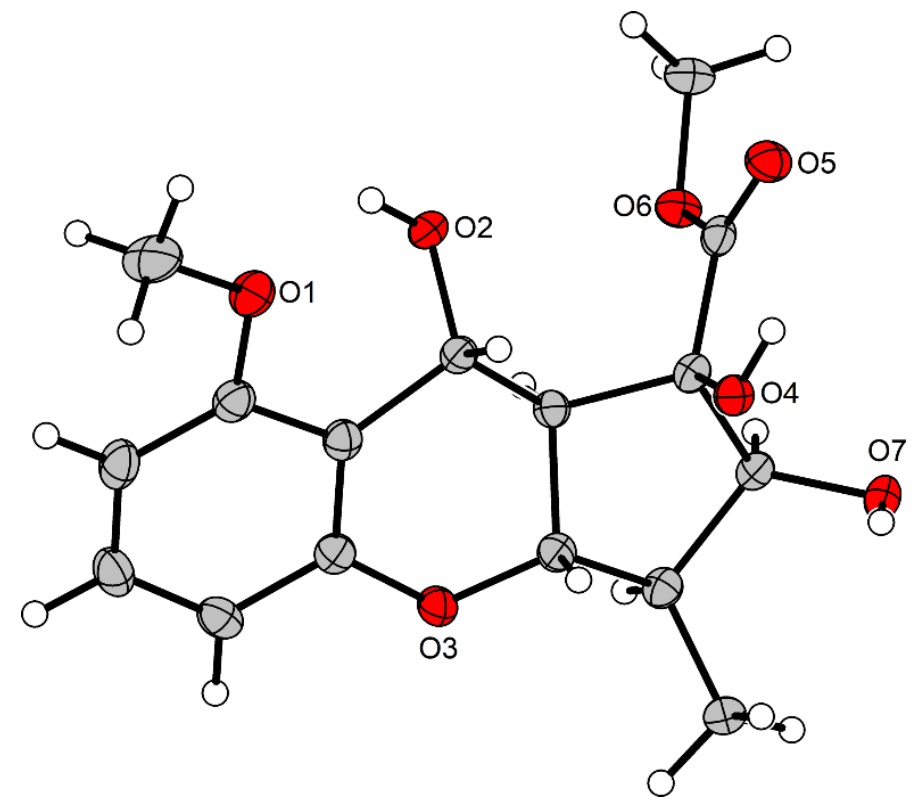

Figure 2. Crystal structure of ent-16. Thermal ellipsoids are shown at 50\% probability.

A suitable crystal of $\mathrm{C}_{16} \mathrm{H}_{20} \mathrm{O}_{7}$ was selected under inert oil and mounted using a MiTeGen loop. Intensity data of the crystal were recorded with a STADIVARI diffractometer (Stoe \& Cie). The diffractometer was operated with $\mathrm{Cu}-\mathrm{K} \alpha$ radiation $(1.54186 \AA$, microfocus source) and equipped with a Dectris PILATUS 300K detector. Evaluation, integration and reduction of the diffraction data was carried out using the X-Area software suite. ${ }^{[4]}$ Multi-scan and numerical absorption corrections were applied with the LANA and X-RED32 modules of the X-Area software suite. ${ }^{[5,6]}$ The structure was solved using dual-space methods (SHELXT-2014/5) and refined against $F^{2}$ (SHELXL-2018/3 using ShelXle interface). ${ }^{[7-9]}$ All non-hydrogen atoms were refined with anisotropic displacement parameters. The hydrogen atoms were refined using the "riding model" approach with isotropic displacement parameters 1.2 times (1.5 times for the methyl groups) of that of the preceding carbon atom. CCDC 2129122 contains the supplementary crystallographic data for this paper. These data can be obtained free of charge from The Cambridge Crystallographic Data Centre via www.ccdc.cam.ac.uk/structures.

Empirical formula

Molar mass / $\mathrm{g} \cdot \mathrm{mol}^{-1}$

Space group (No.)

$a / \AA$

$b / \AA$

$c / \AA$
$\mathrm{C}_{16} \mathrm{H}_{20} \mathrm{O}_{7}$

324.32

$P 2{ }_{1}{ }_{1} 2_{1}$ (19)

6.58400(10)

9.66390(10)

24.5168(3) 
$\rho_{\text {calc. }} / \mathrm{g} \cdot \mathrm{cm}^{-3}$

$\mu / \mathrm{mm}^{-1}$

Color colorless

Crystal habitus

plate

Crystal size $/ \mathrm{mm}^{3}$

$T / \mathrm{K}$

$0.163 \times 0.160 \times 0.048$

$\lambda / \AA$

100

$\theta$ range $1^{\circ}$

$1.54186\left(\mathrm{Cu}-\mathrm{K}_{\alpha}\right)$

Range of Miller indices

3.606 to 76.376

$-8 \leq h \leq 5$

$-12 \leq k \leq 12$

$-29 \leq l \leq 30$

Absorption correction

multi-scan and numerical

$T_{\min }, T_{\max }$

$0.5885,0.9272$

$R_{\text {int }}, R_{\sigma}$

$0.0324,0.0167$

Completeness of the data set

1.000

No. of measured reflections

52299

No. of independent reflections

3249

No. of parameters

221

No. of restraints

0

$S$ (all data)

1.099

$R(F)(I \geq 2 \sigma(I)$, all data $)$

$0.0262,0.0283$

$w R\left(F^{2}\right)(I \geq 2 \sigma(I)$, all data $)$

$0.0694,0.0701$

Extinction coefficient

$0.0016(4)$

Flack parameter $x$

$-0.02(5)$

$\Delta \rho_{\max }, \Delta \rho_{\min } / \mathrm{e} \cdot \AA^{-3}$

$0.227,-0.147$ 


\subsection{Methyl $\quad(1 S, 2 R, 3 R, 3 \mathrm{a} R, 9 R, 9 \mathrm{a} S)$-2-acetoxy-1,9-dihydroxy-8-methoxy-3- methyl-1,2,3,3a,9,9a-hexahydrocyclopenta[b]chromene-1-carboxylate (18b)}

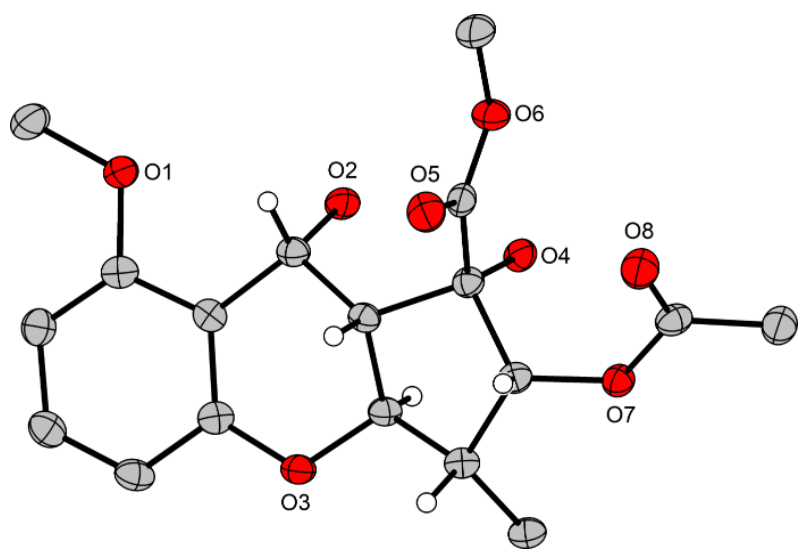

Figure 3. Crystal structure of $\mathbf{1 8 b}$. Thermal ellipsoids are shown at 50\% probability.

A suitable crystal of $\mathrm{C}_{18} \mathrm{H}_{22} \mathrm{O}_{8}$ was selected under inert oil and mounted using a MiTeGen loop. Intensity data of the crystal were recorded with a STADIVARI diffractometer (Stoe \& Cie). The diffractometer was operated with $\mathrm{Cu}-\mathrm{K} \alpha$ radiation $(1.54186 \AA$, microfocus source) and equipped with a Dectris PILATUS 300K detector. Evaluation, integration and reduction of the diffraction data was carried out using the X-Area software suite. ${ }^{[4]}$ Multi-scan and numerical absorption corrections were applied with the LANA and X-RED32 modules of the X-Area software suite. ${ }^{[5,6]}$ The structure was solved using dual-space methods (SHELXT-2018/2) and refined against $F^{2}$ (SHELXL-2018/3 using ShelXle interface). ${ }^{[7-9]}$ All non-hydrogen atoms were refined with anisotropic displacement parameters. The hydrogen atoms were refined using the "riding model" approach with isotropic displacement parameters 1.2 times (1.5 times for the methyl groups) of that of the preceding carbon atom. CCDC 2129124 contains the supplementary crystallographic data for this paper. These data can be obtained free of charge from The Cambridge Crystallographic Data Centre via www.ccdc.cam.ac.uk/structures.

Empirical formula

Molar mass / $\mathrm{g} \cdot \mathrm{mol}^{-1}$

Space group (No.)

$a / \AA$

$b / \AA$

$c / \AA$

$\beta /^{\circ}$

$V / \AA^{3}$

Z

$\rho_{\text {calc. }} / \mathrm{g} \cdot \mathrm{cm}^{-3}$

\section{$\mathrm{C}_{18} \mathrm{H}_{22} \mathrm{O}_{8}$}

366.35

$P 2_{1}$ (4)

9.18700(10)

8.70620(10)

$11.39140(10)$

$110.8050(10)$

$851.718(16)$

1.429 
$\mu / \mathrm{mm}^{-1}$

Color

Crystal habitus

Crystal size $/ \mathrm{mm}^{3}$

$T / \mathrm{K}$

$\lambda / \AA$

$\theta$ range $/^{\circ}$

Range of Miller indices

Absorption correction

$T_{\min }, T_{\max }$

$R_{\text {int }}, R_{\sigma}$

Completeness of the data set

No. of measured reflections

No. of independent reflections

No. of parameters

No. of restraints

$S$ (all data)

$R(F)(I \geq 2 \sigma(I)$, all data)

$w R\left(F^{2}\right)(I \geq 2 \sigma(I)$, all data $)$

Extinction coefficient

Flack parameter $x$

$\Delta \rho_{\max }, \Delta \rho_{\min } / \mathrm{e} \cdot \AA^{-3}$
0.954

colorless

plate

$0.291 \times 0.280 \times 0.114$

100

$1.54186\left(\mathrm{Cu}-\mathrm{K}_{\alpha}\right)$

4.152 to 76.027

$-4 \leq h \leq 11$

$-10 \leq k \leq 10$

$-14 \leq l \leq 14$

multi-scan and numerical

$0.3978,0.8473$

$0.0216,0.0104$

0.998

26074

3471

244

1

1.046

$0.0273,0.0275$

$0.0735,0.0737$

$0.0068(9)$

$-0.03(10)$

$0.228,-0.156$

\section{References}

[1] a) Kitamura, M. Synthesis Of 2-Azido-1,3-dimethylimidazolinium Hexafluorophosphate (ADMP). Org. Synth. 2015, 92, 171-181. b) Kitamura, M.; Tashiro, N.; Miyagawa, S.; Okauchi, T. 2-Azido-1,3-dimethylimidazolinium Salts: Efficient Diazo-Transfer Reagents for 1,3-Dicarbonyl Compounds. Synthesis 2011, 2011, 1037-1044.

[2] Kerste, E.; Beller, M. P.; Koert, U. Second Generation Total Synthesis of (-)Preussochromone D. Eur. J. Org. Chem. 2020, 2020, 3699-3711.

[3] Zhang, F.; Li, L.; Niu, S.; Si, Y.; Guo, L.; Jiang, X.; Che, Y. A thiopyranchromenone and other chromone derivatives from an endolichenic fungus, preussia africana. J. Nat. Prod. 2012, 75, 230-237. 
[4] X-Area, STOE \& Cie GmbH, Darmstadt, Germany, 2018.

[5] LANA - Laue Analyzer, STOE \& Cie GmbH, Darmstadt, Germany, 2019.

[6] X-RED32, STOE \& Cie GmbH, Darmstadt, Germany, 2018.

[7] Sheldrick, G. M. Acta Crystallogr., Sect. A: Found. Adv. 2015, 71, 3-8.

[8] Sheldrick, G. M. Acta Crystallogr., Sect. C: Struct. Chem. 2015, 71, 3-8.

[9] Hübschle, C. B.; Sheldrick, G. M.; Dittrich, B. J. Appl. Crystallogr. 2011, 44, 1281-1284. 